\title{
Chemical Composition of Selected Core Samples, Idaho National Engineering Laboratory, Idaho
}

By LeRoy L. Knobel and L. DeWayne Cecil, U.S. Geological Survey, and Thomas R. Wood, Lockheed Idaho Technologies Company

U.S. GEOLOGICAL SURVEY

Open-File Report 95-748

Prepared in cooperation with

U.S. DEPARTMENT OF ENERGY

Idaho Falls, Idaho

November 1995 


\title{
U.S. DEPARTMENT OF THE INTERIOR \\ BRUCE BABBITT, Secretary
}

\author{
U.S. GEOLOGICAL SURVEY \\ GORDON P. EATON, Director
}

Any use of trade, product, or firm names is for descriptive purposes

only and does not imply endorsement by the U.S. Government.

For additional information write to:

Project Chief

U.S. Geological Survey

INEL, MS 4148

P.O. Box 2230

Idaho Falls, ID 83403
Copies of this report can be purchased from:

U.S. Geological Survey

Earth Science Information Center

Open-File Reports Section

Box 25286, MS 517

Denver Federal Center

Denver, CO 80225 


\section{CONTENTS}

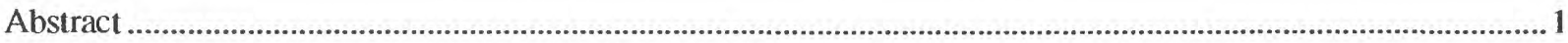

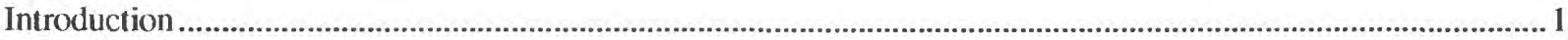

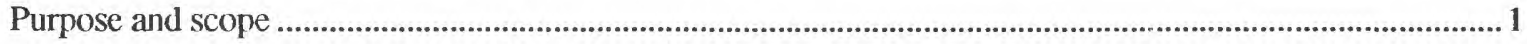

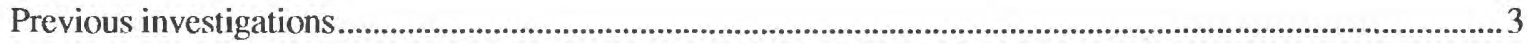

Estimating crustal abundances of major rock-forming elements ......................................................................

Acknowledgments ....................................................................................................................................................

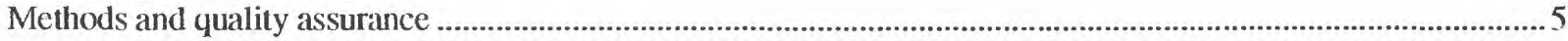

Corehole locations and sample collection .............................................................................................................. 5

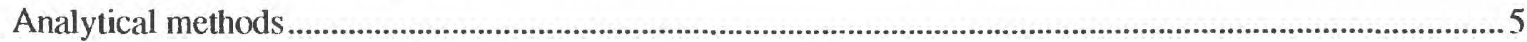

Wavelength dispersive X-ray fluorescence spectrometry ............................................................................5

Inductively coupled plasma mass spectrometry ........................................................................................... 6

Quality assurance ....................................................................................................................................................

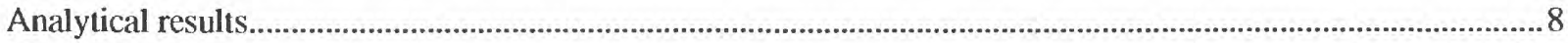

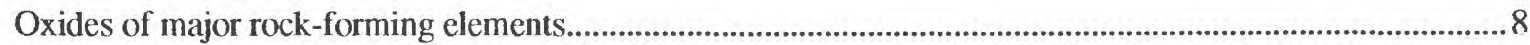

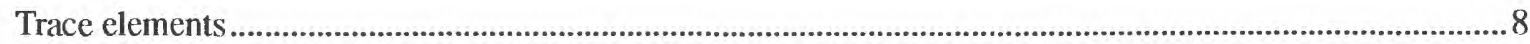

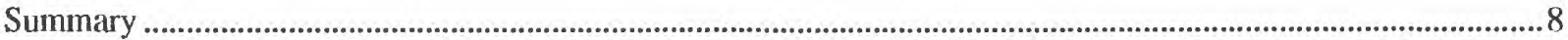

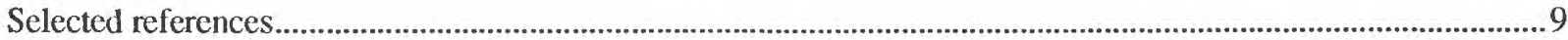

\section{FIGURES}

1. Map showing location of the Idaho National Engineering Laboratory and selected facilities .............................2

2. Map showing location of selected corehole sites at the Idaho National Engineering Laboratory ........................4

\section{TABLES}

1. Chemical composition (percent by weight) of selected core subsamples, by major rock-forming element, Idaho National Engineering Laboratory, Idaho

2. Chemical composition (percent by weight) of selected core subsamples, by corehole and depth, Idaho National Engineering Laboratory, Idaho.

3. Trace-element concentrations of selected core subsamples, by corehole and analytical method, Idaho National Engineering Laboratory, Idaho. 
CONVERSION FACTORS, VERTICAL DATUM, AND ABBREVIATED UNITS

\begin{tabular}{rcc}
\hline Multiply & By & To obtain \\
\hline foot (ft) & 0.3048 & meter \\
inch (in.) & 25.4 & millimeter \\
square mile ( $\left.\mathrm{mi}^{2}\right)$ & 2.590 & square kilometer \\
\hline
\end{tabular}

For temperature, degrees Celsius $\left({ }^{\circ} \mathrm{C}\right)$ can be converted to degrees Fahrenheit $\left({ }^{\circ} \mathrm{F}\right)$ by using the equation: ${ }^{\circ} \mathrm{F}=(1.8)\left({ }^{\circ} \mathrm{C}\right)+32$.

Abbreviated units used in report: $\mathrm{g}$ (gram); $\mathrm{mg}$ (milligram); $\mathrm{mL}$ (milliliter); ppb (part per billion); ppm (part per million). 


\title{
Chemical Composition of Selected Core Samples, Idaho National Engineering Laboratory, Idaho
}

\author{
by LeRoy L. Knobel and L. DeWayne Cecil, U.S. Geological Survey, and \\ Thomas R. Wood, Lockheed Idaho Technologies Company
}

\begin{abstract}
This report presents chemical compositions determined from 84 subsamples and 5 qualityassurance split subsamples of basalt core from the eastern Snake River Plain. The 84 subsamples were collected at selected depths from 5 coreholes located on the Idaho National Engineering Laboratory, Idaho. This report was jointly prepared by Lockheed Idaho Technologies Company and the U.S. Geological Survey, in cooperation with the U.S. Department of Energy, Idaho Operations Office.
\end{abstract}

Ten major elements and as many as 32 trace elements were determined for each subsample either by wavelength dispersive $\mathrm{X}$-ray fluorescence spectrometry, inductively coupled plasma mass spectrometry, or by both methods. Descriptive statistics for each element were calculated and tabulated by analytical method for each corehole.

\section{INTRODUCTION}

The Idaho National Engineering Laboratory (INEL), which encompasses about $890 \mathrm{mi}^{2}$ of the eastern Snake River Plain in southeastern Idaho (fig. 1), is operated by the U.S. Department of Energy (DOE). INEL facilities are used in the development of peacetime atomic-energy applications, nuclear safety research, defense programs, and advanced energy concepts. Liquid radiochemical and chemical wastes generated at these facilities have been discharged to onsite infiltration ponds and disposal wells since 1952. Many of the waste constituents enter the Snake River Plain aquifer indirectly following percolation through the unsaturated zone (Pittman and others, 1988, p. 2).

In 1949, the U.S. Atomic Energy Commission-now the U.S. DOE-requested that the U.S. Geological Survey (USGS) investigate the geohydrologic conditions at the INEL and adjacent areas before the beginning of reactor operations. Ongoing research by the USGS and Lockheed Idaho Technologies Company (LITCO) at the INEL involves investigation of the migration of radioactive elements contained in low-level radioactive waste, hydrologic and geologic factors affecting waste movement, and geochemical factors that influence the chemical composition of the waste. Identification of the chemistry of Snake River Plain aquifer materials is needed to aid in the study of the hydrology and geochemistry of subsurface waste disposal. This report was prepared jointly by LITCO and the USGS in cooperation with the DOE, Idaho Operations Office. Washington State University (WSU) provided analytical services under contract to EG\&G Idaho, Inc.

\section{Purpose and Scope}

The purpose of this report is to provide a public record of archived information describing the chemical composition of geologic materials from the Snake River Plain aquifer. This report describes (1) the major- and trace-element composition of 84 subsamples and 5 quality-assurance split subsamples of basalt core from 5 sites (fig. 2) and (2) the methods used to analyze the samples. The subsamples were collected from continuous cores at the time of coring or shortly afterward. The remaining cores subsequently were archived at the U.S. Geological Survey’s lithologic-core 


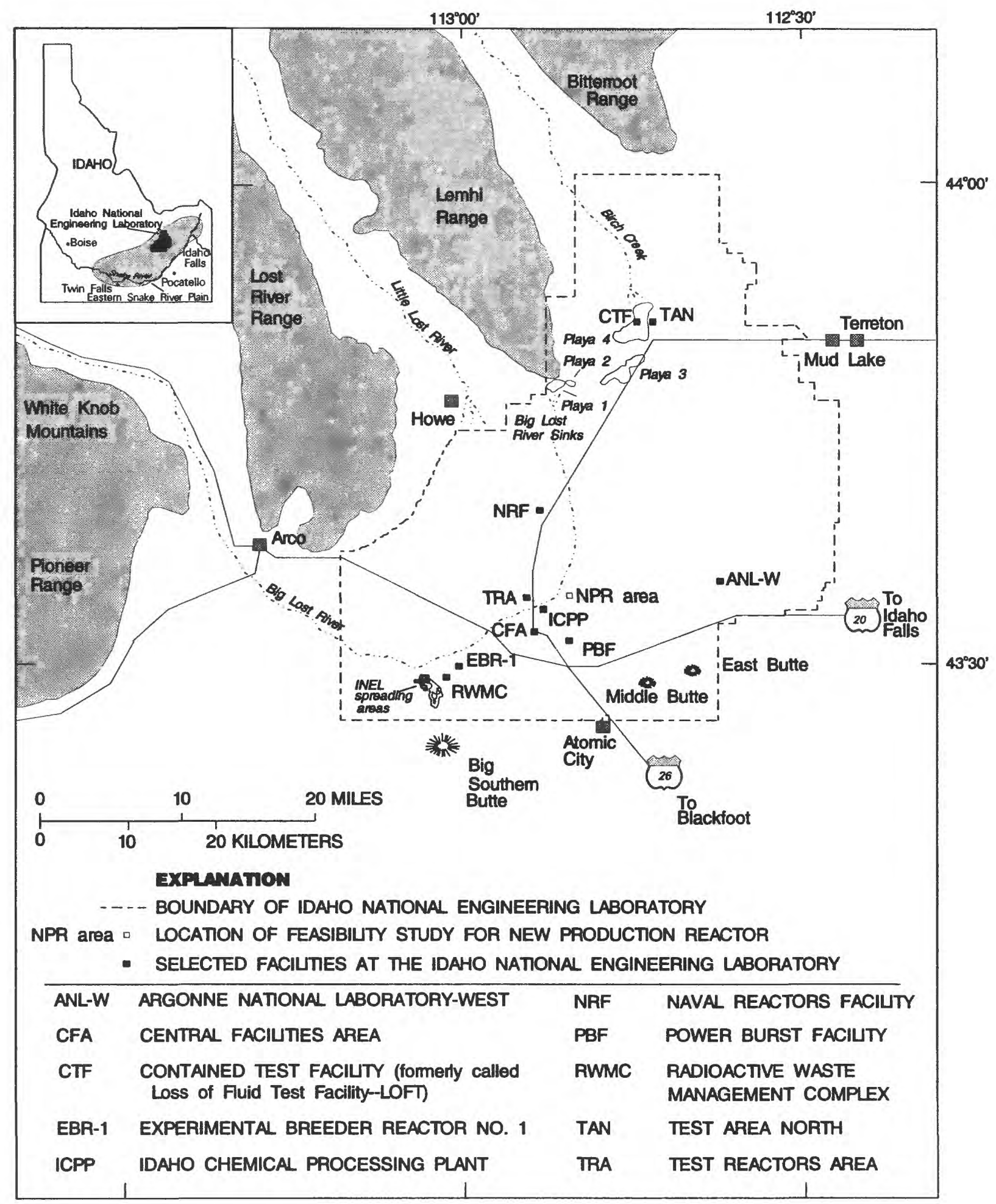

Figure 1.-Location of the Idaho National Engineering Laboratory and selected facilities. 
library at the INEL and are available for additional study

\section{Previous Investigations}

Geologic, hydrologic, and water-quality investigations have been conducted at the INEL since it was selected as a reactor-testing station in 1949. Nace and others (1956) and Nace and others (1975) reported major rock-forming element and selected trace-element chemistry of a basalt sample from Craters of the Moon National Monument and a nephelite basalt sample from the Fort Hall Indian Reservation. Jones (1961) reported the average composition of major rock-forming elements in 38 basalt samples from the Snake River Plain. The data of Nace and others $(1956 ; 1975)$ and Jones (1961) were republished by Bartholomay and others (1989).

Kuntz and Dalrymple (1979) determined major rock-forming element chemistry for 17 basalt samples from large volcanoes in the eastern Snake River Plain and compared the average concentrations to those of various tholeiitic basalts from other locations. Fiesinger and others (1982) analyzed 26 basaltic lava samples from an area southeast of the eastern Snake River Plain for major rock-forming elements and compared results to basaltic lava compositions of samples from the Snake River Plain.

Leeman (1982a) provided major rock-forming element chemistry of 22 representative hybrid lava samples from the Snake River Plain. Leeman (1982b) also summarized major rock-forming element and selected trace-element chemistry for basalt samples from the Snake River Plain and discussed two major variants of basaltic lavas that are present on the Snake River Plain. Honjo and Leeman (1987) reported major- and trace-element chemistry for selected samples of hybrid ferrolatite lavas from the Snake River Plain. The chemical data were selected from a more detailed report about the petrology and geochemistry of the Magic Reservoir eruptive center in south-central Idaho (Honjo, 1986).

Wood and Low (1988) reported average compositions of major rock-forming elements for groups of 152 and 15 basalt samples from the
Snake River Plain. They also provided specific analytical results for 13 basalt samples from the Snake River Plain.

Shervais and others (1994) reported ranges of major-element oxide compositions and minor- and trace-element concentrations for 59 rock samples from 2 corcholes at the INEL. The rock samples represented 14 major flow groups that are part of the castern Snake River Plain aquifer system.

\section{Estimating Crustal Abundances of Major Rock-Forming Elements.}

Historical estimates of average crustal abundances of major rock-forming elements by Clarke and Washington (1924) were computed from about 5,000 available analyses of igneous rocks that they felt were of superior quality; igneous rocks are the predominant rock type of the Earth's crust. Averages of geographically grouped rock analyses agreed fairly well with one another, which indicates regional similarity in the composition of the Earth's crust. The overall average composition computed by Clarke and Washington (1924) is intermediate between that of granite and basalt (Mason, 1966, p. 42).

Mason (1966) pointed out several objections to computing average compositions of igneous rocks by averaging analyses. The first objection was that the geographical distribution of analyses was uneven because the most intensive investigations had taken place in North America and Europe; therefore, results of averaging tended to downplay the importance of other continental areas and the occanic basins. The second objection was that undue weight was given to rare and unusual rock types, which are studied more frequently than more common igneous rocks such as basalt and granite. The third objection was that all analyses were given equal weight regardless of the areas or volumes occupied by the respective rock types.

Goldschmidt (1954) minimized some of these objections by using representative samples of the Earth's crust to compute the average composition. The samples were mostly crystalline rocks derived from the widely distributed glacial clay in southern Norway. This clay was formed from a fine rock flour deposited by meltwater from the 


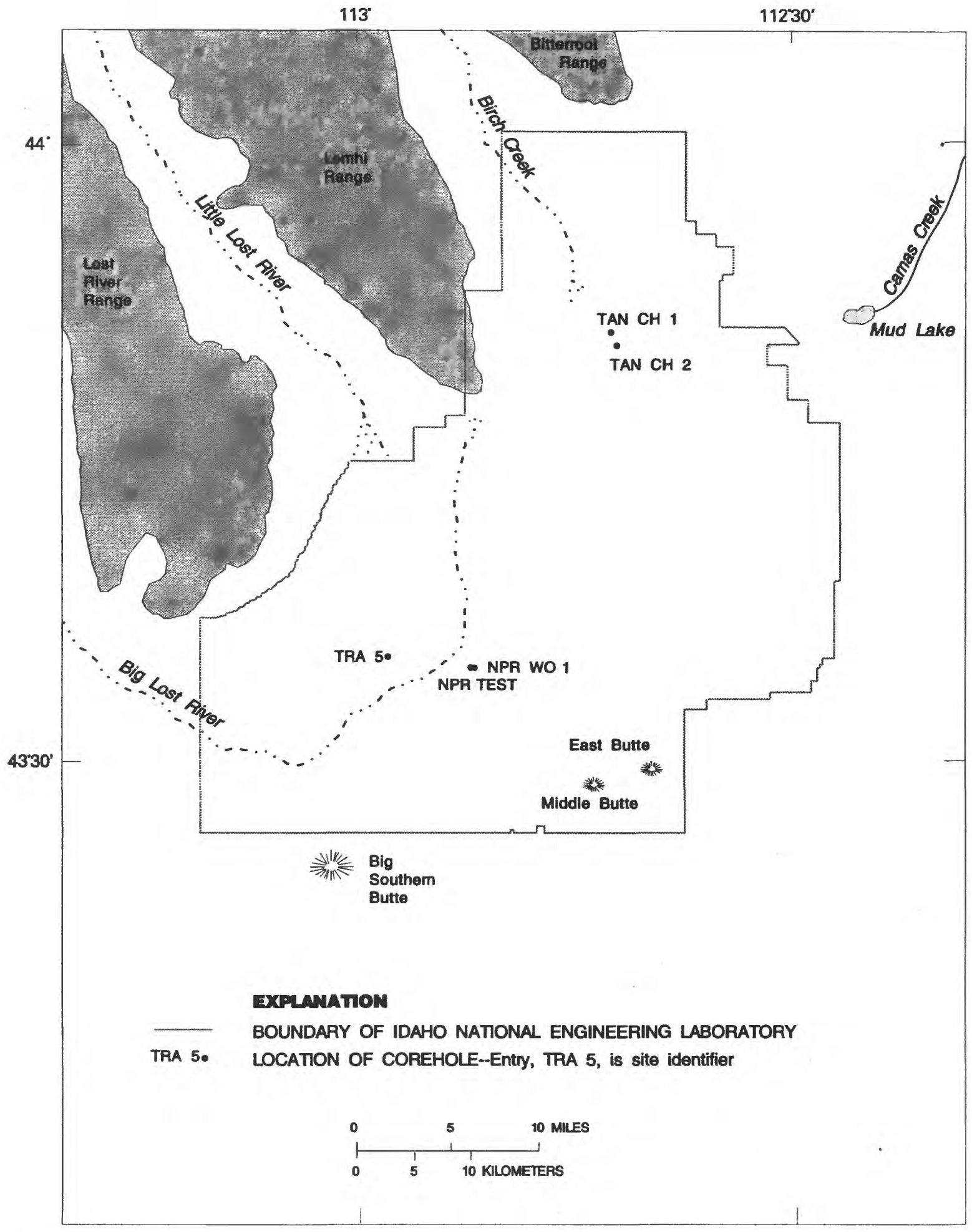

Figure 2.-Location of selected corehole sites at the Idaho National Engineering Laboratory. 
Fennoscandian ice sheet. Analyses from 77 samples were used to calculate an average composition similar to the one computed by Clarke and Washington (1924). Neither of these average compositions accounted for all the objections noted by Mason (1966), especially the one related to not sampling the oceanic basins.

Poldervaart (1955) resolved these objections more completely by computing averages by major geologic type: dcep oceanic regions, continental shields, young folded belts, and continental platforms and slopes. The amount of the major rock types and their estimated compositions were used in computing final average crustal compositions. Because this technique gives adequate consideration to the oceanic crustal material, it more accurately reflects the true average composition of the crust (Mason, 1966). The average compositions of major rock-forming elements calculated by Poldervaart (1955) are the average crustal abundances reported in table 1 .

Average crustal abundances of trace elements listed in table 3 are from Emsley (1989). The average crustal abundances in this report are for comparison purposes only and do not necessarily represent the average compositions of the aquifer materials from the Snake River Plain listed in this report. Additional information regarding crustal abundances of elements is provided by Clarke (1924), Clarke and Washington (1924), Goldschmidt (1954), Poldervaart (1955), Mason (1966), Fyfe (1974), and Greenwood and Earnshaw (1984).

\section{Acknowledgments}

The authors are grateful to Peter R. Hooper of WSU, David L. Naftz of the USGS, and Robert C. Starr of LITCO for technically reviewing the manuscript.

\section{METHODS AND QUALITY ASSURANCE}

The methods used for subsampling core samples, for analyzing the subsamples in the laboratory, and for quality assurance are outlined in the following sections.

\section{Corehole Locations and Sample Collection}

Core samples were collected by EG\&G Idaho, Inc. at five sites (fig. 2): two near Test Area North (TAN CH 1 and TAN CH 2), two near the New Production Reactor site (NPR Test and NPR WO1), and one near the Test Reactors Area (TRA 5). Completion dates for the coreholes follow: TAN CH 1 (September 27, 1989), TAN CH 2 (September 6, 1990), NPR Test (May 6, 1984), NPR WO1 (August 30, 1990), and TRA 5 (September 18, 1990). Corehole TRA 5 subsequently was completed as a piezometer and currently is identified as PZ1.

Eighty-four subsamples of cores were collected from the 5 sites (at selected depths) distributed as follows: 35 subsamples from corchole TAN CH 2, 18 subsamples from corehole TAN CH 1, 21 subsamples from corehole NPR Test, 4 subsamples from corehole NPR WO1, and 6 subsamples from corehole TRA 5. The subsampling technique involved removing a 1- to 3in.-long section of fresh core from a specific depth determined on the basis of representative lithology, labeling the subsample, and storing the subsample until shipment to the WSU GeoAnalytical Laboratory at Pullman, Wash., for analysis.

\section{Analytical Methods}

Two separate analytical methods were used to determine elemental compositions of the subsamples: wavelength dispersive $\mathrm{X}$-ray fluorescence spectrometry (XRF) and inductively coupled plasma mass spectrometry (ICP/MS).

\section{Wavelength Dispersive X-ray Fluorescence Spectrometry}

XRF was used to analyze all 84 subsamples and 2 quality-assurance split subsamples for 10 major rock-forming elements and 17 trace elements. GeoAnalytical Laboratory staff handpicked about $28 \mathrm{~g}$ of fresh chips from each subsample with the largest dimension measuring about 0.4 in. The chips were processed for 2 minutes in a Tema swingmill with tungsten carbide surfaces. Part of the resulting powder $(3.5 \mathrm{~g})$ and $7 \mathrm{~g}$ of lithium tetraborate were mixed for 10 minutes in a plastic mixing jar with an enclosed plastic ball. The mixed 
powder was emptied into graphite crucibles, which were loaded, 24 at a time, into a muffle furnace that was preheated to $1,000^{\circ} \mathrm{C}$. The mixed powder was fused for 5 minutes after the temperature in the furnace returned to $1,000^{\circ} \mathrm{C}$. The crucibles were removed from the furnace and allowed to cool for 10 minutes. Each resulting glass bead was reground in the Tema swingmill for 35 seconds. The resulting glass powder was returned to the crucible and refused for an additional 5 minutes at $1,000^{\circ} \mathrm{C}$. The beads then were labeled with an engraver. The lower flat surface of each bead was ground on a 600-grit grinding wheel, finished on a glass plate (600 grit with alcohol) to remove metal residue from the grinding wheel, washed in an ultrasonic cleaner, rinsed in alcohol, and dried.

The glass beads were analyzed by XRF using a Rigaku 3370 automated wavelength spectrometer. Concentrations of 27 elements in the subsamples were measured by comparing $\mathrm{X}$-ray intensities for each element in the subsample bead with intensities in duplicate beads for 8 international standards and the values recommended by Govindaraju (1989). Specific operating conditions at the WSU GeoAnalytical Laboratory, precision data, and estimates of accuracy for each element are provided by Hooper and others (1993, tables 4-6).

Hooper and others (1993) discussed methodrelated uncertainties for the GeoAnalytical Laboratory. Niobium concentrations less than 2 ppm can be affected by contamination from the tungsten carbide mills; however, hecause of the larger niobium concentrations in INEL samples, the effect should be minimal.

The subsample preparation process causes volatiles to be lost during fusing. Neither loss on ignition (LOI) nor the ferric/ferrous iron ratio was measured by the WSU laboratory. As a result, the totals listed in table 2 cannot be used as a measure of analytical accuracy, as is customary in gravimetric analyses. However, the divergence of any single subsample from 100 percent reflects the variation in volatile content relative to average values for the eight standards used. Thus, if the totals in table 2 are greater than 100 percent, the subsamples had less volatile content than the average for the standards. If the totals in table 2 are less than 100 percent, the converse is true. The most common cause for volatile content less than 100 percent (more than the average value for standards) is that most rocks contain some ferric iron and the WSU laboratory reports all iron as ferrous iron.

Trace-element concentrations of barium, chromium, nickel, scandium, and vanadium that are less than $30 \mathrm{ppm}$ are considered semiquantitative by the WSU laboratory. A few samples listed in table 3 contained concentrations of nickel and scandium less than $30 \mathrm{ppm}$ and these values should be considered tentative. Similarly, trace element concentrations of niobium, rubidium, strontium, thorium, yttrium, and zirconium that are less than 3 ppm are considered semiquantitative. In table 3,1 rubidium concentration and 65 thorium concentrations were less than $3 \mathrm{ppm}$. Lanthanum and cerium XRF concentrations are considered qualitative hy the WSU laboratory and should not be compared to ICP/MS concentrations. Other trace-element concentrations measured by XRF are generally satisfactory, although there is some question about copper, gallium, lead, and zinc measurements. Additional information is available for XRF analytical precision and accuracy measurements in a report by Hooper and others (1993).

\section{Inductively Coupled Plasma Mass Spectrometry}

ICP/MS was used to analyze 56 of the 84 subsamples and 3 quality assurance split subsamples for 22 trace clements. GeoAnalytical Lahoratory staff completely dissolved the core subsamples prior to analysis. The subsamples were ground in an iron bowl in a shatterbox swing mill. A fraction of the powdered subsample weighing $2 \mathrm{~g}$ was mixed with an equal amount of lithium tetraborate flux and loaded into a muffle furnace that was preheated to $1,000^{\circ} \mathrm{C}$. The mixture was fused for 5 minutes after the furnace returned to $1,(0) 0^{\circ} \mathrm{C}$. The resulting head was briefly ground in the shatterbox swing mill. Two hundred fifty mg of the resulting powder was dissolved in an open Teflon vial on a hotplate at $110^{\circ} \mathrm{C}$ with $6 \mathrm{~mL}$ of concentrated hydrolluoric acid (HF), $2 \mathrm{~mL}$ of concentrated nitric acid $\left(\mathrm{HNO}_{3}\right)$, and $2 \mathrm{~mL}$ of concentrated perchloric acid $\left(\mathrm{HClO}_{4}\right)$. The sub- 
sample then was evaporated until dry, redissolved with $2 \mathrm{~mL}$ of concentrated $\mathrm{HClO}_{4}$, and evaporated again. This procedure converted insoluble fluorides to soluble perchlorates. An additional $3 \mathrm{~mL}$ of concentrated $\mathrm{HNO}_{3}, 8$ drops of concentrated hydrogen peroxide $\left(\mathrm{H}_{2} \mathrm{O}_{2}\right), 10$ drops of concentrated $\mathrm{HF}$, an internal standard of indium and rhenium, and enough distilled water to make a final volume of $60 \mathrm{~mL}$ were added to the subsample. This procedure removed silica and boron matrix interferences by volatilizing them as gaseous fluorides and ensured complete dissolution of refractory phases such as zircon and garnet (Knaack and others, 1994).

The subsamples were analyzed by ICP/MS using a Sciex Elan model 250 inductively coupled plasma mass spectrometer equipped with a Babington nebulizer, a water cooled spray chamber, and Brooks mass flow controllers. Subsamples were introduced by peristaltic pump into an argon plasma at $1.0 \mathrm{~mL}$ per minute using an automatic sampler. Plasma power was 1,500 watts, which generates a plasma temperature of $7,000^{\circ} \mathrm{C}$. These conditions minimize the formation of metal oxides. The instrument can measure many elements during each sample run. For each element, a count time of 0.5 seconds is repeated 10 times for a total integrated count time per clement of 5 seconds. For elements with more than 1 isotope, target isotope selection is based on relative isotope abundance and freedom from interferences. The quadrupole mass spectrometer with an inductively coupled argon plasma ion source used by WSU's GeoAnalytical Laboratory is capable of quantitatively determining trace elements in liquids in the concentration range of fractions of a part per billion (Knaack and others, 1994). Duplicatc subsamples from 3 laboratory rock standards, 1 acid blank sample, and 17 unknown subsamples were run together forming a total of 24 standard and unknown subsamples per batch. The 3 laboratory standards have been calibrated against 11 international standards by the GeoAnalytical Laboratory and elemental concentrations have been independently verified by other laboratories. Additional information for ICP/MS analytical precision and accuracy is available from the WSU GeoAnalytical Laboratory (Knaack and others, 1994).

\section{Quality Assurance}

Five quality-assurance subsamples were analyzed for elemental concentrations by the WSU GeoAnalytical Laboratory. The samples are identified in tables $1-3$ by the letter "R" adjacent to the depth entry.

XRF--Replicate beads were prepared by the GeoAnalytical Laboratory for splits of the 36-ft subsample from the NPR Test corchole and the 448-ft subsample from the NPR WO1 corelole. These replicate heads were analyzed for the same 27 elements as the reference sample heads. Percentage differences between pairs of analytical results were calculated for the 36 - and 448-ft subsamples. For the 10 major rock-forming elements listed in tables 1 and 2, all analytical results for the replicate beads were within 10 percent difference of the reference sample beads. For the 17 trace elements listed in table 3 that were analyzed by XRF, analytical results for the replicate beads were within 10 percent difference of the reference sample beads for 9 clements. The other 8 elements (cerium, copper, gallium, lanthanum, lead, rubidium, thorium, and zinc) had percentage differences greater than 10 percent. Of these, Hooper and others (1993) identified cerium and lanthanum concentrations as being strictly qualitative measurements. Copper, gallium, lead, and zinc concentrations were identified as questionable in some cases. The calculated percentage differences for rubidium and thorium concentrations that exceed 10 percent were for subsample pairs that had concentrations near the 3 ppm cutoff where the XRF method becomes semiquantitative.

ICP/MS.-Replicate beads also were prepared by the GeoAnalytical Laboratory for splits of the 923-ft subsample from the TAN CH 2 corchole and the 195- and 593-ft subsamples from the TAN CH 1 corehole. These replicate beads were analyzed for the same 22 elements as the reference subsample beads. Percentage differences for all but onc replicate subsample bead were within 10 percent of the reference subsample beads. The 923-ft replicate subsample bead for the TAN CH 2 corehole had a 12.2-percent difference from the reference subsample bead for uranium. 
ICP/MS-XRF Comparison.-Because of overlap between analytical methods, 7 elements were determined by both analytical methods for 56 of the 84 subsamples. Percentage differences were calculated between analytical results from the two methods for concentrations of each element, by depth, using the ICP/MS concentration as the reference concentration. The percentage differences were calculated by subtracting the XRF value from the ICP/MS value, dividing hy the $\mathrm{ICP} / \mathrm{MS}$ value, and multiplying hy one hundred.

The seven elements determined using both methods and the ranges of percentage differences for each follow: barium $(+1$ to +20 percent $)$, cerium $(-50$ to +40 percent), lanthanum $(-47$ to +100 percent $)$, lead $(-129$ to +72 percent $)$, niobium $(-21$ to +12 percent $)$, thorium $(-367$ to +100 percent $)$, and yttrium $(0$ to +14 percent $)$. These data indicate that direct comparison of results from the two methods is not valid.

\section{ANALYTICAL RESULTS}

Major rock-forming element composition was determined by XRF analysis and trace element concentrations were determined by XRF and ICP/MS analysis for selected core subsamples. Descriptive statistics were calculated and are reported along with the data in tables 1 and 3 . The errors associated with the mean and median values are standard errors and are an estimate of the uncertainty of the mean and median concentrations. Spiegel (1975, p. 162) published equations for calculation of the standard errors for the mean and median values. The standard errors of the mean and median values are valid if the distribution of a sample approximates a normal distribution. Spiegel (1975) indicated that for sample sizes greater than or equal to 30 , the sampling distribution approximates a normal distribution, even if the population distribution is not normally distributed. Because the sample sizes for the TAN CH 1 and NPR Test coreholes are less than 30 and may not approximate a normal distribution, the standard errors associated with the mean and median values for those data sets should be used with caution.

\section{Oxides of Major Rock-Forming Elements}

Silicon, aluminum, titanium, iron, manganese, calcium, magnesium, potassium, sodium, and phosphorus were determined by XRF analysis for 84 subsamples of core taken from 5 locations. Chemical composition of the samples is reported in terms of elemiental oxides in units of percent by weight. The results are reported in table 1 by elemental oxide, corehole from which subsample was collected, and subsample depth. In the solid phase, iron occurs in both ferrous and ferric form. In this report, all iron is assumed to be ferrous iron and is reported as FeO. Also included for each element in table 1 are average crustal abundances and descriptive statistics.

Table 2 is a listing of the 10 major rockforming elements, in weight percent, by the corehole from which the subsample was collected, and hy subsample depth. For each depth, the 10 weight percents were summed and the totals also are listed in table 2 .

\section{Trace Elements}

As many as 32 trace-element concentrations were determined for 84 subsamples of core taken from 5 locations by either XRF analysis, ICP/MS analysis, or both methods. The results are reported in table 3 by element, corehole from which subsample was collected, subsample depth, and analytical method. Also included for each element in table 3 are average crustal abundances and descriptive statistics.

\section{SUMMARY}

This report presents chemical compositions determined from 84 subsamples and 5 qualityassurance split subsamples of basalt core from the eastem Snake River Plain. The 84 subsamples were collected at selected depths from 5 coreholes located on the Idaho National Engineering Laboratory, Idaho. This report was prepared jointly by LITCO and the USGS, in cooperation with the DOE, Idaho Opcrations Office. WSU provided analytical services under contract to EG\&G Idaho, Inc. 
Ten major elements and as many as .32 trace elements were determined for each subsample either by XRF analysis, ICP/MS analysis, or by both methods. Descriptive statistics for each element were calculated and tabulated by analytical method for each corehole.

\section{SELECTED REFERENCES}

Bartholomay, R.C., Knohel, L.L., and Davis, L.C., 1989, Mineralogy and grain size of surficial sediment from the Big Lost River drainage and vicinity, with chemical and physical characteristics of geologic materials from selected sites at the Idaho National Engneering Laboratory, Idaho: U.S. Geological Survey Open-File Report 89-384 (DOE/ID22081), 74 p.

Clarke. F.W., 1924, The data of geochemistry: U.S. Geological Survey Bulletin 770, 841 p.

Clarke, F.W., and Washington, H.S., 1924, The composition of the Earth's crust: U.S. Geological Survey Professional Paper 127, $117 \mathrm{p}$.

Emsley, John, 1989, The elements: Oxford, Clarendon Press, 256 p.

Fiesinger, D.W., Perkins, W.D., and Puchy, G.J., 1982, Mineralogy and petrology of TertiaryQuaternary volcanic rocks in Caribou County, Idaho, in Bonnichsen, Bill, and Breckenridge, R.M., eds., Cenozoic geology of Idaho: Moscow, Idaho Bureau of Mines and Geology Bulletin 26, p. 465-488.

Fyfe, W.S., 1974, Geochemistry: Oxford, Oxford University Press, 109 p.

Goldschmidt, V.M., 1954 Geochemistry: Oxford Clarendon Press, 730 p.

Govindaraju, 1989, 1989 compilation of working values and sample description for 272 geostandards: Geostandards Newsletter, v. 13, special issue, p. 1-113.

Greenwood, N.N., and Eamshaw, A., 1984. Chemistry of the elements: Oxford, Pergamon Press, 730 p.
Honjo, Norio, 1986, Petrology and geochemistry of the Magic Reservoir eruptive center, Snake River Plain, Idaho: Houston, Tex., Rice University, M.A. Thesis, 511 p.

Honjo, Norio, and Leeman, W.P., 1987, Origin of hybrid ferrolatite lavas from Magic

Reservoir eruptive center, Snake River Plain, Idaho: Contributions to Mineralogy and Petrology, v. 96, p. 163-177.

Hooper, P.R., Johnson, D.M., and Conrey, R.M., 1993, Major and trace element analyses of rocks and minerals by automated X-ray spectrometry: Pullman, Wash., Washington State University Geology Department Open File Report, 12 p.

Jones, P.H., 1961, Hydrology of waste disposal, National Reactor Testing Station, Idaho, an interim report: U.S. Atomic Energy Commission Publication (IDO-22042), 151 p.

Knaack, Charles, Cornelius, Scott, and Hooper, Peter, 1994, Trace element analyses of rocks and minerals by ICP-MS: Pullman, Wash., Washington State University Geology Department Open File Report, variously paginated.

Kuntz, M.A., and Dalrymple, G.B., 1979, Geology, geochronology, and potential volcanic hazards in the Lava Ridge-Hells Half Acre area, eastern Snake River Plain, Idaho: U.S. Geological Survey Open-File Report 79-1657, 66 p.

Leeman, W.P., 1982a, Evolved and hybrid lavas from the Snake River Plain, Idaho, in Bonnichsen, Bill, and Breckenridge, R.M., eds., Cenozoic geology of Idaho: Moscow, Idaho Bureau of Mines and Geology Bulletin 26, p. 193-202.

----1982b, Olivine tholeiitic basalts of the Snake River Plain, Idaho, in Bonnichsen, Bill, and Breckenridge, R.M., eds., Cenozoic geology of Idaho: Moscow, Idaho Bureau of Mines and Geology Bulletin 26, p. 181-191.

Mason, Brian, 1966, Principles of geochemistry: New York, John Wiley \& Sons, Inc., 329 p. 
Nace, R.L., Deutsch, Morris, and Voegeli, P.T., 1956, Geography, geology, and water resources of the National Reactor Testing Station, Idaho, Part 2: Geography and geology: U.S. Atomic Energy Commission Publication (IDO-22033), 225 p.

Nace, R.L., Voegeli, P.T., Jones, J.R., and Deutsch, Morris, 1975, Generalized geologic framework of the National Reactor Testing Station, Idaho: U.S. Geological Survey Professional Paper 725-B, 49 p.

Pittman, J.R., Jensen, R.J., and Fischer, P.R., 1988, Hydrologic conditions at the Idaho National Engineering Laboratory, 1982 to 1985: U.S. Geological Survey WaterResources Investigations Report 89-40018 (DOE/ID-22078), 73 p.

Poldervaart, A., ed., 1955, Crust of the earth: Geologic Society of America Special Paper $62,762 \mathrm{p}$.

Shervais, John, Vetter, Scott, and Hackett, William, 1994, Chemical stratigraphy of basalts in coreholes NPR-E and WO-2, Idaho National Engineering Laboratory, Idaho: Implications for plume dynamics in the eastem Snake River Plain [Abs.]:

International Symposium on the Observation of the Continental Crust Through Drilling, VIIth, Santa Fe, N. Mex., April 25-30, 1994, [Proceedings], unpaginated.

Spiegel, M.R., 1975, Probability and statistics: New York, McGraw-Hill Book Co., 372 p.

Wood, W.W., and Low, W.H., 1988, Solute geochemistry of the Snake River Plain regional aquifer system, Idaho and eastern Oregon: U.S. Geological Survey Professional Paper 1408-D, 79 p. 
Table 1.-Chemical composition (percent by weight) of selected core subsamples, by major rock-forming element, Idaho National Engineering Laboratory, Idaho

[Location of coreholes is shown on figure 2. Units for depth are feet below land surface.

Abbreviations: TAN, Test Area North; CH, corehole; NPR, New Production Reactor; TRA, Test Reactors Area; XRF, wavelength-dispersive X-ray fluorescence spectrometry. Analyses by Washington State University. Depth: R indicates quality-assurance split subsample of the specified sample depth. Quality-assurance samples were not included in calculation of statistical parameters. Percent by weight data for oxides in this report were not normalized except for average crustal abundances, which were normalized to a water-free basis by Poldervaart (1955). In this report, total iron is expressed as simple ferrous iron oxide (FeO). Mason (1966) discussed the limitations of estimating crustal oxide abundances of major rock-forming elements and summarized the results of Poldervaart (1955)]

\section{ELEMENTS}

$\begin{array}{llll}\text { A: } & \text { Silicon } & \text { F: } & \text { Calcium } \\ \text { B: } & \text { Aluminum } & \text { G: } & \text { Magnesium } \\ \text { C: } & \text { Titanium } & \text { H: } & \text { Potassium } \\ \text { D: } & \text { Iron } & \text { I: } & \text { Sodium } \\ \text { E: } & \text { Manganese } & \text { J: } & \text { Phosphorus }\end{array}$


A: Silicon oxide $\left(\mathrm{SiO}_{2}\right)$-Average crustal abundance is 55.2 percent by weight (Mason, 1966)

\begin{tabular}{|c|c|c|c|c|c|c|c|}
\hline \multicolumn{8}{|c|}{ [Oxide composition of core sample] } \\
\hline \multicolumn{2}{|c|}{ TAN CH 2} & \multicolumn{2}{|c|}{ TAN CH 1} & \multicolumn{2}{|c|}{ NPR Test } & \multicolumn{2}{|c|}{ NPR W01 } \\
\hline Depth & $\overline{X R F}$ & Depth & $\overline{\text { XRF }}$ & Depth & $\overline{\text { XRF }}$ & Depth & $\overline{\text { XRF }}$ \\
\hline 138 & 45.56 & 61 & 46.29 & 36 & 46.35 & 418 & 47.43 \\
\hline 154 & 45.63 & 91 & 45.76 & $36 \mathrm{R}$ & 46.81 & 448 & 47.53 \\
\hline 172 & 46.09 & 111 & 46.06 & 56 & 45.96 & $448 \mathrm{R}$ & 47.78 \\
\hline 201 & 45.33 & 130 & 45.51 & 98 & 46.95 & 476 & 47.02 \\
\hline 215 & 46.56 & 150 & 46.78 & 121 & 47.90 & 522 & 47.06 \\
\hline 238 & 45.33 & 195 & 48.18 & 127 & 47.50 & & \\
\hline 256 & 47.89 & 250 & 47.54 & 141 & 47.31 & & \\
\hline 277 & 46.61 & 300 & 46.77 & 156 & 47.26 & \multicolumn{2}{|c|}{ TRA 5} \\
\hline 299 & 47.71 & 380 & 46.60 & 170 & 47.58 & Depth & $\overline{\text { XRF }}$ \\
\hline 309 & 46.77 & 421 & 47.23 & 232 & 47.16 & 100 & 47.21 \\
\hline 327 & 47.12 & 435 & 46.39 & 273 & 46.83 & 139 & 47.06 \\
\hline 352 & 46.96 & 460 & 46.88 & 291 & 46.64 & 162 & 47.73 \\
\hline 366 & 46.51 & 475 & 46.37 & 311 & 47.49 & 170 & 46.71 \\
\hline 413 & 46.22 & 491 & 45.91 & 379 & 47.43 & 192 & 46.99 \\
\hline 463 & 46.84 & 500 & 46.62 & 450 & 47.11 & 202 & 46.71 \\
\hline 477 & 45.89 & 521 & 46.23 & 461 & 47.01 & & \\
\hline 487 & 46.86 & 571 & 46.29 & 480 & 47.28 & & \\
\hline 490 & 46.84 & 593 & 45.95 & 489 & 47.72 & & \\
\hline 521 & 47.56 & & & 527 & 47.64 & & \\
\hline 545 & 46.10 & & & 538 & 47.74 & & \\
\hline 576 & 46.25 & & & 550 & 47.86 & & \\
\hline 592 & 46.04 & & & 608 & 46.67 & & \\
\hline 630 & 46.28 & & & & & & \\
\hline 659 & 46.38 & & & & & & \\
\hline 673 & 46.19 & & & & & & \\
\hline 730 & 46.48 & & & & & & \\
\hline 752 & 47.10 & & & & & & \\
\hline 785 & 46.20 & & & & & & \\
\hline 816 & 46.22 & & & & & & \\
\hline 832 & 47.97 & & & & & & \\
\hline 870 & 47.29 & & & & & & \\
\hline 886 & 46.89 & & & & & & \\
\hline 923 & 46.56 & & & & & & \\
\hline 961 & 47.09 & & & & & & \\
\hline 990 & 46.42 & & & & & & \\
\hline
\end{tabular}

[Descriptive statistics]

\begin{tabular}{|c|c|c|c|c|}
\hline & TAN CH 2 & TAN CH 1 & NPR Test & All cores \\
\hline Parameter & XRF & XRF & XRF & XRF \\
\hline Minimum & 45.33 & 45.51 & 45.96 & 45.33 \\
\hline Maximum & 47.97 & 48.18 & 47.90 & 48.18 \\
\hline Mean & 46.56 & 46.52 & 47.21 & 46.78 \\
\hline Error & .11 & .15 & .11 & .07 \\
\hline Median & 46.51 & 46.38 & 47.28 & 46.805 \\
\hline Error & .14 & .19 & .14 & .09 \\
\hline Sample size & 35 & 18 & 21 & 84 \\
\hline
\end{tabular}


B: Aluminum oxide $\left(\mathrm{Al}_{2} \mathrm{O}_{3}\right)$-Average crustal abundance is 15.3 percent by weight (Mason, 1966)

\begin{tabular}{|c|c|c|c|c|c|c|c|}
\hline \multicolumn{8}{|c|}{ [Oxide composition of core sample] } \\
\hline \multicolumn{2}{|c|}{ TAN CH 2} & \multicolumn{2}{|c|}{ TAN $\mathrm{CH} 1$} & \multicolumn{2}{|c|}{ NPR Test } & \multicolumn{2}{|c|}{ NPR W01 } \\
\hline Depth & $\overline{X R F}$ & Depth & XRF & Depth & XRF & Depth & XRF \\
\hline 138 & 13.29 & 61 & 13.06 & 36 & 14.62 & 418 & 15.87 \\
\hline 154 & 13.64 & 91 & 14.15 & $36 \mathrm{R}$ & 14.66 & 448 & 16.27 \\
\hline 172 & 13.80 & 111 & 14.27 & 56 & 14.55 & $448 \mathrm{R}$ & 16.31 \\
\hline 201 & 13.40 & 130 & 13.90 & 98 & 14.60 & 476 & 14.54 \\
\hline 215 & 13.99 & 150 & 13.81 & 121 & 14.86 & 522 & 15.63 \\
\hline 238 & 13.74 & 19.5 & 14.58 & 127 & 14.86 & & \\
\hline 256 & 14.99 & 250 & 14.96 & 141 & 14.55 & & \\
\hline 277 & 14.64 & 300 & 14.54 & 156 & 14.95 & \multicolumn{2}{|c|}{ TRA 5} \\
\hline 299 & 13.87 & 380 & 14.82 & 170 & 14.93 & Depth & $\overline{\text { XRF }}$ \\
\hline 309 & 14.60 & 421 & 15.25 & 232 & 15.00 & 100 & 15.34 \\
\hline 327 & 14.65 & 435 & 15.09 & 273 & 14.34 & 139 & 15.11 \\
\hline 352 & 14.64 & 460 & 14.79 & 291 & 15.26 & 162 & 15.10 \\
\hline 366 & 14.83 & 47.5 & 14.84 & 311 & 15.31 & 170 & 15.06 \\
\hline 413 & 14.84 & 491 & 14.29 & 379 & 15.52 & 192 & 14.25 \\
\hline 463 & 15.26 & 500 & 14.52 & 450 & 16.26 & 202 & 13.67 \\
\hline 477 & 14.57 & 521 & 14.86 & 461 & 14.76 & & \\
\hline 487 & 14.27 & 571 & 14.85 & 480 & 11.80 & & \\
\hline 490 & 14.90 & 593 & 14.66 & 489 & 15.07 & & \\
\hline 521 & 13.71 & & & 527 & 15.37 & & \\
\hline 545 & 14.49 & & & 538 & 15.51 & & \\
\hline 576 & 15.08 & & & 550 & 15.47 & & \\
\hline 592 & 14.52 & & & 608 & 14.45 & & \\
\hline 6.30 & 14.85 & & & & & & \\
\hline 659 & 14.49 & & & & & & \\
\hline 673 & 14.67 & & & & & & \\
\hline 730 & 14.60 & & & & & & \\
\hline 752 & 13.59 & & & & & & \\
\hline 785 & 14.06 & & & & & & \\
\hline 816 & 13.88 & & & & & & \\
\hline 832 & 14.04 & & & & & & \\
\hline 870 & 13.95 & & & & & & \\
\hline 886 & 14.44 & & & & & & \\
\hline 923 & 14.51 & & & & & & \\
\hline 961 & 14.76 & & & & & & \\
\hline 990 & 14.46 & & & & & & \\
\hline
\end{tabular}

[Descriptive statistics]

\begin{tabular}{|c|c|c|c|c|}
\hline & TAN CH 2 & TAN CH 1 & NPR Test & All cores \\
\hline Parameter & $\overline{X A F}$ & $\overline{\mathrm{XRF}}$ & XRF & XRF \\
\hline Minimum & 13.29 & 13.06 & 11.80 & 11.80 \\
\hline Maximum & 15.26 & 15.25 & 16.26 & 16.27 \\
\hline Mean & 14.34 & 14.51 & 14.86 & 14.60 \\
\hline Error & .09 & .13 & .18 & .07 \\
\hline Median & 14.49 & 14.62 & 14.93 & 14.63 \\
\hline Error & .11 & .16 & .23 & .09 \\
\hline Sample size & 35 & 18 & 21 & 84 \\
\hline
\end{tabular}


C: Titanium oxide $\left(\mathrm{TiO}_{2}\right)$ - Average crustal abundance is 1.6 percent by weight (Mason, 1966)

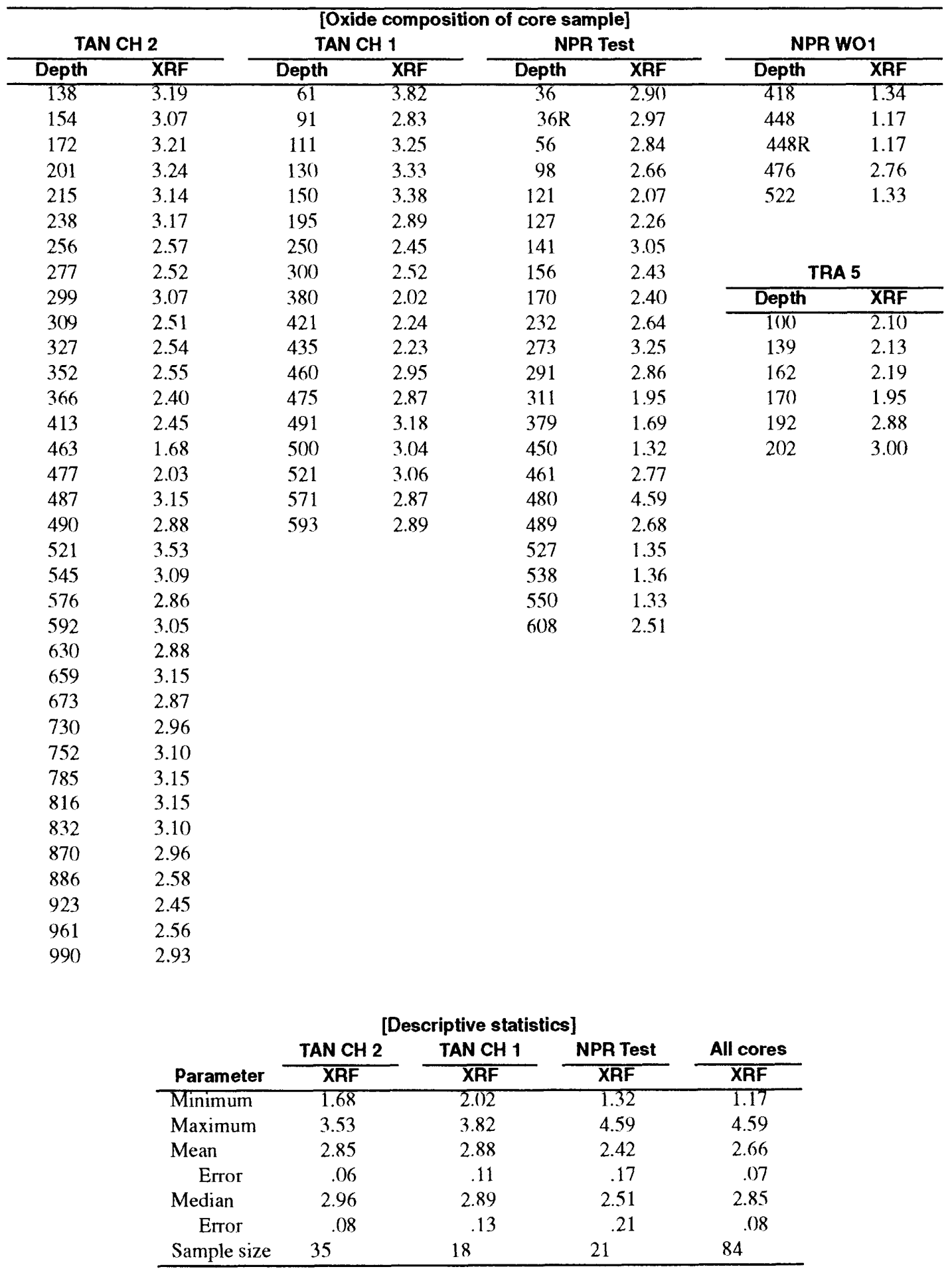


D: Iron oxide (FeO)-Average crustal abundance is 8.6 percent by weight (Mason, 1966)

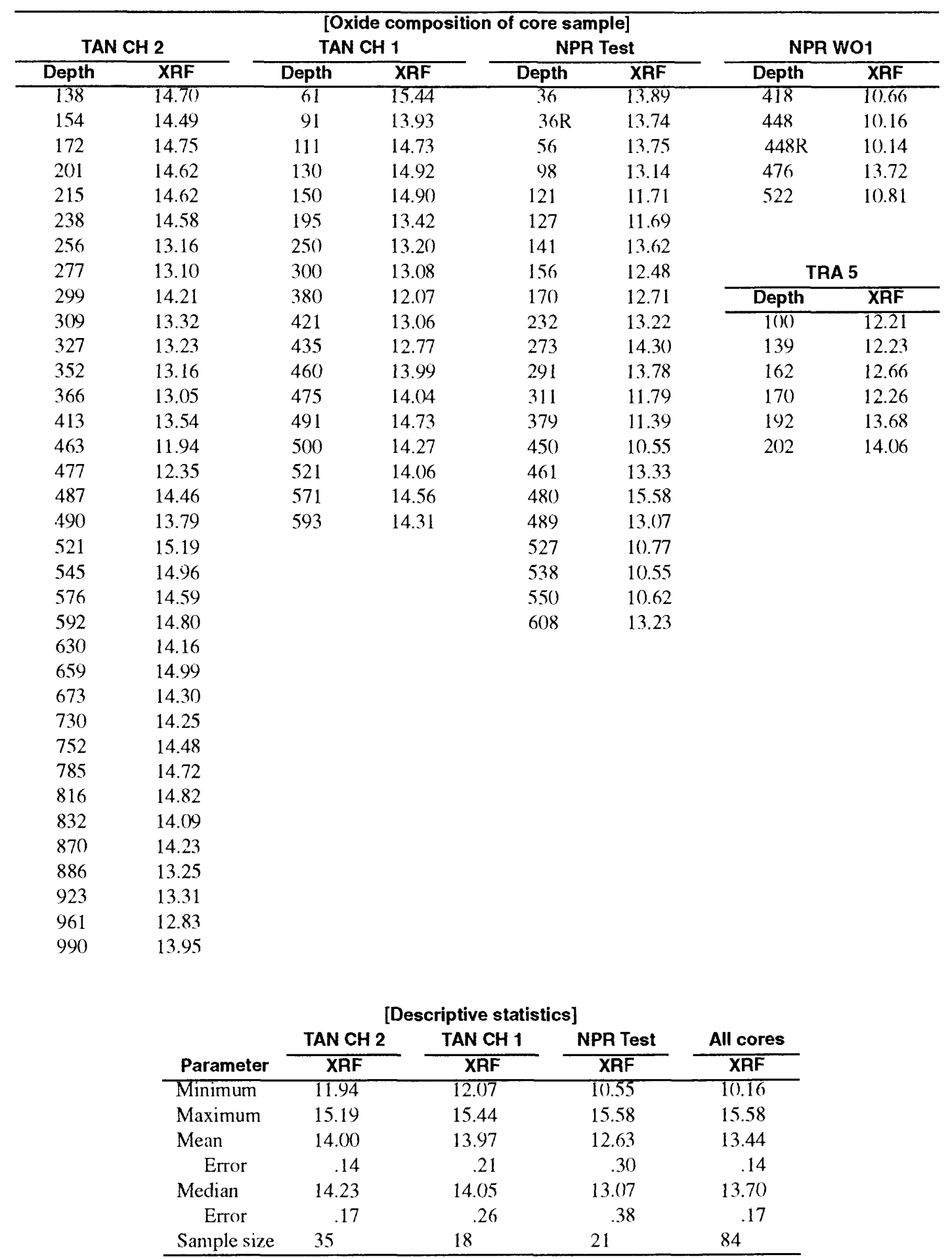


E: Manganese oxide (MnO) - Average crustal abundance is 0.2 percent by weight (Mason, 1966)

\begin{tabular}{|c|c|c|c|c|c|c|c|}
\hline \multicolumn{8}{|c|}{ [Oxide composition of core sample] } \\
\hline \multicolumn{2}{|c|}{ TAN CH 2} & \multicolumn{2}{|c|}{ TAN CH 1} & \multicolumn{2}{|c|}{ NPR Test } & \multicolumn{2}{|c|}{ NPR W01 } \\
\hline Depth & $\overline{\text { XRF }}$ & Depth & XRF & Depth & XRF & Depth & XRF \\
\hline 138 & 0.22 & 61 & 0.23 & 36 & 0.20 & 418 & 0.18 \\
\hline 154 & .22 & 91 & .21 & $36 \mathrm{R}$ & .20 & 448 & .17 \\
\hline 172 & .21 & 111 & .22 & 56 & .20 & $448 \mathrm{R}$ & .17 \\
\hline 201 & .22 & 130 & .22 & 98 & $.2 i)$ & 476 & .20 \\
\hline 215 & .22 & 150 & .22 & 121 & .18 & 522 & .18 \\
\hline 238 & .21 & 195 & .21 & 127 & .19 & & \\
\hline 256 & .20 & 250 & .20 & 141 & .21 & & \\
\hline 277 & .20 & 300 & .19 & 156 & .19 & \multicolumn{2}{|c|}{ TRA 5} \\
\hline 299 & .21 & 380 & .19 & 170 & .19 & Depth & XRF \\
\hline 309 & .20 & 421 & .20 & 232 & .20 & 100 & 0.19 \\
\hline 327 & .20 & 435 & .19 & 273 & .21 & 139 & .19 \\
\hline 352 & .20 & 460 & .20 & 291 & .20 & 162 & .19 \\
\hline 366 & .19 & 475 & .20 & 311 & .19 & 170 & .19 \\
\hline 413 & .19 & 491 & .21 & 379 & .19 & 192 & .21 \\
\hline 463 & .18 & 500 & .21 & 450 & .18 & 202 & .21 \\
\hline 477 & .19 & 521 & .21 & 461 & .20 & & \\
\hline 487 & .21 & 571 & .21 & 480 & .23 & & \\
\hline 490 & .20 & 593 & .20 & 489 & .20 & & \\
\hline 521 & .22 & & & 527 & .17 & & \\
\hline 545 & .21 & & & 538 & .17 & & \\
\hline 576 & .20 & & & 550 & .18 & & \\
\hline 592 & .21 & & & 608 & .20 & & \\
\hline 630 & .20 & & & & & & \\
\hline 659 & .21 & & & & & & \\
\hline 673 & .21 & & & & & & \\
\hline 730 & .20 & & & & & & \\
\hline 752 & .22 & & & & & & \\
\hline 785 & .21 & & & & & & \\
\hline 816 & .22 & & & & & & \\
\hline 832 & .21 & & & & & & \\
\hline 870 & .21 & & & & & & \\
\hline 886 & .20 & & & & & & \\
\hline 923 & .20 & & & & & & \\
\hline 961 & .19 & & & & & & \\
\hline 990 & .20 & & & & & & \\
\hline
\end{tabular}

[Descriptive statistics]

\begin{tabular}{|c|c|c|c|c|}
\hline & TAN CH 2 & TAN CH 1 & NPR Test & All cores \\
\hline Parameter & XRF & XRF & $\overline{X R F}$ & $\overline{\mathrm{XRF}}$ \\
\hline Minimum & 0.19 & 0.19 & 0.17 & 0.17 \\
\hline Maximum & .22 & .23 & .23 & .23 \\
\hline Mean & .21 & .21 & .19 & .20 \\
\hline Error & .002 & .003 & .003 & .001 \\
\hline Median & .21 & .21 & .20 & .20 \\
\hline Error & .002 & .003 & .004 & .002 \\
\hline Sample size & 35 & 18 & 21 & 84 \\
\hline
\end{tabular}


F: Calcium oxide $(\mathrm{CaO})$-Average crustal abundance is 8.8 percent by weight (Mason, 1966)

\begin{tabular}{|c|c|c|c|c|c|c|c|}
\hline \multicolumn{8}{|c|}{ [Oxide composition of core sample] } \\
\hline \multicolumn{2}{|c|}{ TAN CH 2} & \multicolumn{2}{|c|}{ TAN CH 1} & \multicolumn{2}{|c|}{ NPR Test } & \multicolumn{2}{|c|}{ NPR W01 } \\
\hline Depth & XRF & Depth & XRF & Depth & XAF & Depth & XRF \\
\hline 138 & 9.58 & 61 & 10.18 & $\overline{36}$ & 9.45 & 418 & 11.51 \\
\hline 154 & 9.53 & 91 & 9.50 & $36 \mathrm{R}$ & 9.53 & 448 & 11.29 \\
\hline 172 & 9.42 & 111 & 9.72 & 56 & 9.39 & $448 \mathrm{R}$ & 11.32 \\
\hline 201 & 9.38 & 130 & 9.63 & 98 & 9.46 & 476 & 9.52 \\
\hline 215 & 9.36 & 150 & 9.59 & 121 & 9.94 & 522 & 10.48 \\
\hline 238 & 9.48 & 195 & 9.41 & 127 & 10.09 & & \\
\hline 256 & 9.29 & 250 & 9.40 & 141 & 9.43 & & \\
\hline 277 & 9.34 & 300 & 9.41 & 156 & 9.33 & \multicolumn{2}{|c|}{ TRA 5} \\
\hline 299 & 9.45 & 380 & 10.10 & 170 & 9.35 & Depth & $\overline{\text { XRF }}$ \\
\hline 309 & 9.35 & 421 & 10.48 & 232 & 9.54 & 100 & 9.87 \\
\hline 327 & 9.40 & 435 & 10.46 & 273 & 9.61 & 139 & 9.79 \\
\hline 352 & 9.65 & 460 & 9.31 & 291 & 9.66 & 162 & 10.04 \\
\hline 366 & 9.62 & 475 & 9.24 & 311 & 10.45 & 170 & 9.67 \\
\hline 413 & 9.74 & 491 & 9.39 & 379 & 10.81 & 192 & 10.23 \\
\hline 463 & 10.40 & 500 & 9.35 & 450 & 11.64 & 202 & 9.63 \\
\hline 477 & 9.95 & 521 & 9.67 & 461 & 9.61 & & \\
\hline 487 & 9.25 & 571 & 9.54 & 480 & 9.42 & & \\
\hline 490 & 9.24 & 593 & 9.52 & 489 & 9.68 & & \\
\hline 521 & 9.34 & & & 527 & 10.36 & & \\
\hline 545 & 9.38 & & & 538 & 10.54 & & \\
\hline 576 & 9.57 & & . & 550 & 10.64 & & \\
\hline 592 & 9.61 & & & 608 & 10.16 & & \\
\hline 630 & 9.55 & & & & & & \\
\hline 659 & 9.78 & & & & & & \\
\hline 673 & 9.46 & & & & & & \\
\hline 730 & 9.57 & & & & & & \\
\hline 752 & 9.44 & & & & & & \\
\hline 785 & 9.62 & & & & & & \\
\hline 816 & 9.41 & & & & & & \\
\hline 832 & 9.36 & & & & & & \\
\hline 870 & 9.36 & & & & & & \\
\hline 886 & 9.82 & & & & & & \\
\hline 923 & 9.64 & & & & & & \\
\hline 961 & 10.15 & & & & & & \\
\hline 990 & 9.80 & & & & & & \\
\hline
\end{tabular}

\begin{tabular}{|c|c|c|c|c|}
\hline \multicolumn{5}{|c|}{ [Descriptive statistics] } \\
\hline & TAN CH 2 & TAN CH 1 & NPR Test & All cores \\
\hline Parameter & XRF & $\overline{\text { XRF }}$ & $\overline{\mathrm{XRF}}$ & $\overline{\text { XRF }}$ \\
\hline Minimum & 9.24 & 9.24 & 9.33 & 9.24 \\
\hline Maximum & 10.40 & 10.48 & 11.64 & 11.64 \\
\hline Mean & 9.55 & 9.66 & 9.93 & 9.75 \\
\hline Error & .04 & .09 & .13 & .05 \\
\hline Median & 9.48 & 9.53 & 9.66 & 9.585 \\
\hline Error & .05 & .11 & .17 & .07 \\
\hline Sample size & 35 & 18 & 21 & 84 \\
\hline
\end{tabular}


G: Magnesium oxide ( $\mathrm{MgO}$ )—Average crustal abundance is 5.2 percent by weight (Mason, 1966)

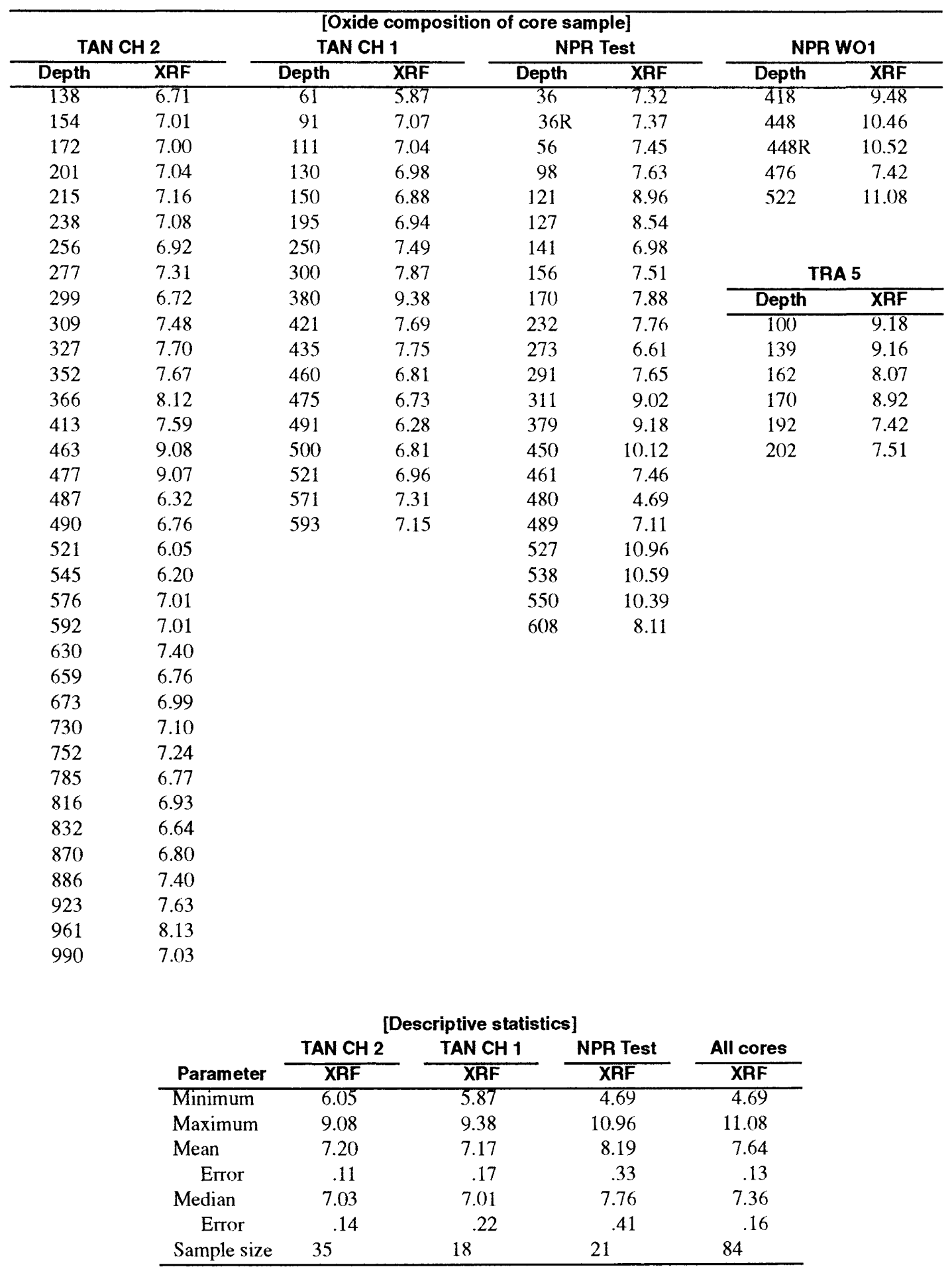


$\mathrm{H}$ : Potassium oxide $\left(\mathrm{K}_{2} \mathrm{O}\right)$ - Average crustal abundance is 1.9 percent by weight (Mason, 1966)

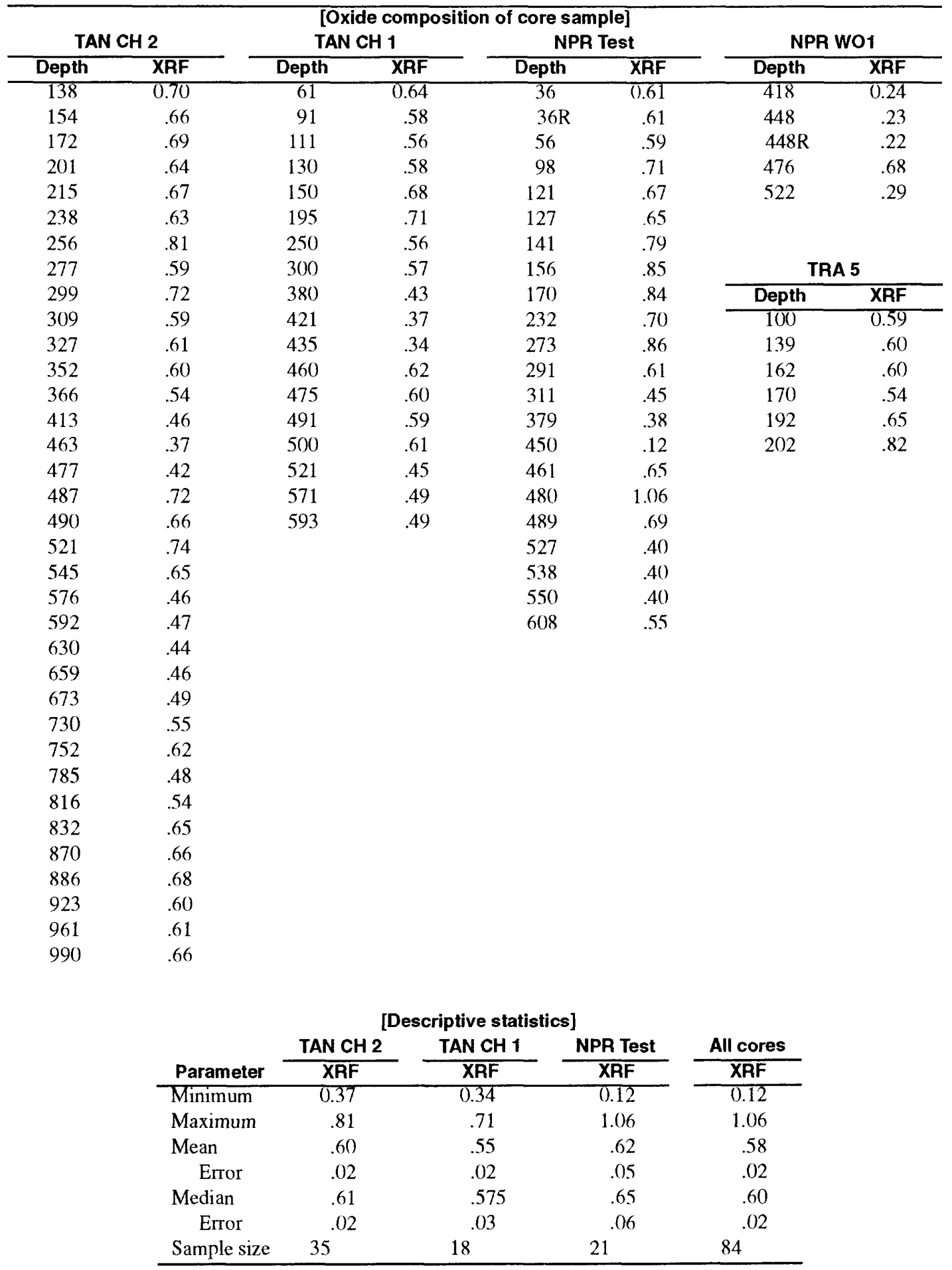


I: Sodium oxide $\left(\mathrm{Na}_{2} \mathrm{O}\right)$ - Average crustal abundance is 2.9 percent by weight (Mason, 1966)

\begin{tabular}{|c|c|c|c|c|c|c|c|}
\hline \multicolumn{8}{|c|}{ [Oxide composition of core sample] } \\
\hline \multicolumn{2}{|c|}{ TAN CH 2} & \multicolumn{2}{|c|}{ TAN CH 1} & \multicolumn{2}{|c|}{ NPR Test } & \multicolumn{2}{|c|}{ NPR Wo1 } \\
\hline Depth & XRF & Depth & $\overline{X R F}$ & Depth & XRF & Depth & XRF \\
\hline 138 & 2.50 & 61 & 2.61 & 36 & 2.77 & 418 & 2.32 \\
\hline 154 & 2.50 & 91 & 2.58 & $36 \mathrm{R}$ & 2.78 & 448 & 2.29 \\
\hline 172 & 2.56 & 111 & 2.57 & 56 & 2.71 & $448 \mathrm{R}$ & 2.28 \\
\hline 201 & 2.52 & 130 & 2.55 & 98 & 2.75 & 476 & 2.60 \\
\hline 215 & 2.66 & 150 & 2.58 & 121 & 2.53 & 522 & 2.18 \\
\hline 238 & 2.53 & 195 & 2.68 & 127 & 2.55 & & \\
\hline 256 & 2.71 & 250 & 2.70 & 141 & 2.75 & & \\
\hline 277 & 2.64 & 300 & 2.59 & 156 & 2.73 & \multicolumn{2}{|c|}{ TRA 5} \\
\hline 299 & 2.76 & 380 & 2.48 & 170 & 2.67 & Depth & $\overline{\text { XRF }}$ \\
\hline 309 & 2.65 & 421 & 2.55 & 232 & 2.75 & 100 & 2.54 \\
\hline 327 & 2.61 & 435 & 2.50 & 273 & 2.68 & 139 & 2.56 \\
\hline 352 & 2.58 & 460 & 2.76 & 291 & 2.66 & 162 & 2.59 \\
\hline 366 & 2.64 & 475 & 2.80 & 311 & 2.42 & 170 & 2.58 \\
\hline 413 & 2.54 & 491 & 2.67 & 379 & 2.44 & 192 & 2.60 \\
\hline 463 & 2.31 & 500 & 2.74 & 450 & 2.25 & 202 & 2.64 \\
\hline 477 & 2.45 & 521 & 2.75 & 461 & 2.54 & & \\
\hline 487 & 2.81 & 571 & 2.78 & 480 & 2.73 & & \\
\hline 490 & 2.81 & 593 & 2.69 & 489 & 2.62 & & \\
\hline 521 & 2.93 & & & 527 & 2.26 & & \\
\hline 545 & 2.79 & & & 538 & 2.22 & & \\
\hline 576 & 2.78 & & & 550 & 2.25 & & \\
\hline 592 & 2.86 & & & 608 & 2.56 & & \\
\hline 630 & 2.80 & & & & & & \\
\hline 659 & 2.81 & & & & & & \\
\hline 673 & 2.88 & & & & & & \\
\hline 730 & 2.81 & & & & & & \\
\hline 752 & 2.45 & & & & & & \\
\hline 785 & 2.61 & & & & & & \\
\hline 816 & 2.52 & & & & & & \\
\hline 832 & 2.59 & & & & & & \\
\hline 870 & 2.49 & & & & & & \\
\hline 886 & 2.52 & & & & & & \\
\hline 923 & 2.61 & & & & & & \\
\hline 961 & 2.53 & & & & & & \\
\hline 990 & 2.64 & & & & & & \\
\hline
\end{tabular}

[Descriptive statistics]

\begin{tabular}{|c|c|c|c|c|}
\hline & TAN CH 2 & TAN CH 1 & NPR Test & All cores \\
\hline Parameter & XRF & XRF & XRF & XRF \\
\hline Minimum & 2.31 & 2.48 & 2.22 & 2.18 \\
\hline Maximum & 2.93 & 2.80 & 2.77 & 2.93 \\
\hline Mean & 2.64 & 2.64 & 2.56 & 2.60 \\
\hline Error & .02 & .02 & .04 & .02 \\
\hline Median & 2.61 & 2.64 & 2.62 & 2.605 \\
\hline Error & .03 & .03 & .05 & .02 \\
\hline Sample size & 35 & 18 & 21 & 84 \\
\hline
\end{tabular}


$\mathrm{J}$ : Phosphorus oxide $\left(\mathrm{P}_{2} \mathrm{O}_{5}\right)$--Average crustal abundance is 0.3 percent by weight (Mason, 1966)

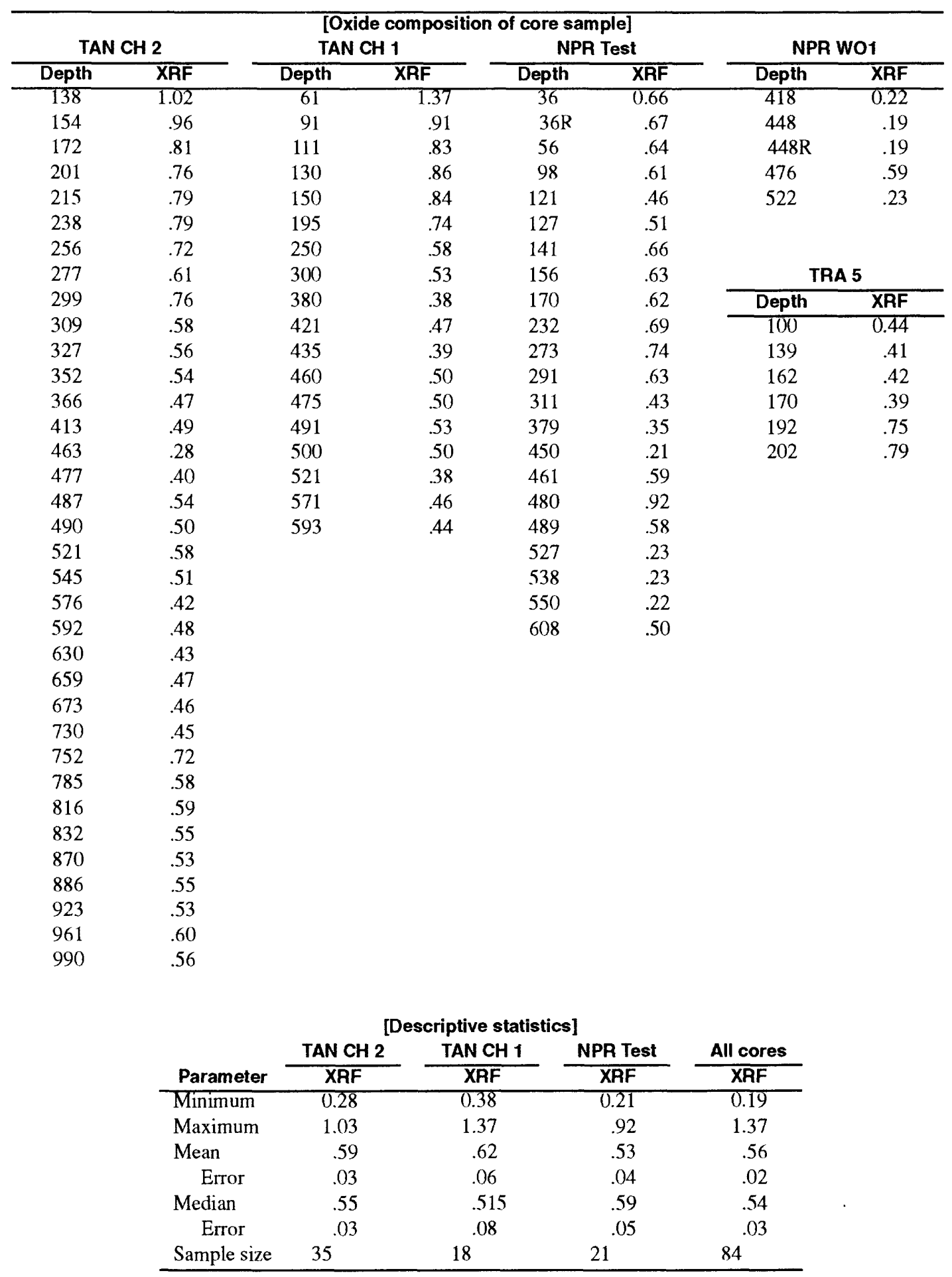


Table 2.-Chemical composition (percent by weight) of selected core subsamples, by corehole and depth, Idaho National Engineering Laboratory, Idaho

[Location of coreholes is shown on figure 2. Units for depth are feet below land surface.

Abbreviations: TAN, Test Area North; CH, corehole; NPR, New Production Reactor; TRA, Test Reactors Area. Analyses by Washington State University using wavelength-dispersive X-ray fluorescence spectrometry. Depth: $\mathrm{R}$ indicates a quality-assurance split subsample of the specified sample depth. In this report, total iron is expressed as simple ferrous iron oxide (FeO). Total indicates the sum of unnormalized oxide chemical compositions by depth]

\section{COREHOLES}
A: $\quad$ TAN CH 2
C: $\quad$ NPR Test and NPR WO1
B: $\quad$ TAN CH 1
D: $\quad$ TRA 5

SYMBOLS FOR CHEMICAL NOTATIONS USED IN TABLE 2

$\begin{array}{llll}\mathrm{SiO}_{2} & \text { Silicon oxide } & \mathrm{CaO} & \text { Calcium oxide } \\ \mathrm{Al}_{2} \mathrm{O}_{3} & \text { Aluminum oxide } & \mathrm{MgO} & \text { Magnesium oxide } \\ \mathrm{TiO}_{2} & \text { Titanium oxide } & \mathrm{K}_{2} \mathrm{O} & \text { Potassium oxide } \\ \mathrm{FeO} & \text { Iron oxide } & \mathrm{Na}_{2} \mathrm{O} & \text { Sodium oxide } \\ \mathrm{MnO} & \text { Manganese oxide } & \mathrm{P}_{2} \mathrm{O}_{5} & \text { Phosphorus oxide }\end{array}$




\section{A: TAN CH 2}

\begin{tabular}{|c|c|c|c|c|c|c|c|c|c|}
\hline \multirow[b]{2}{*}{ Oxide } & \multicolumn{9}{|c|}{ Depth } \\
\hline & 138 & 154 & 172 & 201 & 215 & 238 & 256 & 277 & 299 \\
\hline $\mathrm{SiO}_{2}$ & 45.56 & 45.63 & 46.09 & 45.33 & 46.56 & 45.33 & 47.89 & 46.61 & 47.71 \\
\hline $\mathrm{Al}_{2} \mathrm{O}_{3}$ & 13.29 & 13.64 & 13.80 & 13.40 & 13.99 & 13.74 & 14.99 & 14.64 & 13.87 \\
\hline $\mathrm{TiO}_{2}$ & 3.19 & 3.07 & 3.21 & 3.24 & 3.14 & 3.17 & 2.57 & 2.52 & 3.07 \\
\hline $\mathrm{FeO}$ & 14.70 & 14.49 & 14.75 & 14.62 & 14.62 & 14.58 & 13.16 & 13.10 & 14.21 \\
\hline $\mathrm{MnO}$ & .22 & .22 & .21 & .22 & .22 & .21 & .20 & .20 & .21 \\
\hline $\mathrm{CaO}$ & 9.58 & 9.53 & 9.42 & 9.38 & 9.36 & 9.48 & 9.29 & 9.34 & 9.45 \\
\hline $\mathrm{MgO}$ & 6.71 & 7.01 & 7.00 & 7.04 & 7.16 & 7.08 & 6.92 & 7.31 & 6.72 \\
\hline $\mathrm{K}_{2} \mathrm{O}$ & .70 & .66 & .69 & .64 & .67 & .63 & .81 & .59 & .72 \\
\hline $\mathrm{Na}_{2} \mathrm{O}$ & 2.50 & 2.50 & 2.56 & 2.52 & 2.66 & 2.53 & 2.71 & 2.64 & 2.76 \\
\hline $\mathrm{P}_{2} \mathrm{O}_{5}$ & 1.02 & .96 & .81 & .76 & .79 & .79 & .72 & .61 & .76 \\
\hline Total & 97.47 & 97.71 & 98.54 & 97.15 & 99.17 & 97.54 & 99.26 & 97.56 & 99.48 \\
\hline Oxide & 309 & 327 & 352 & 366 & 413 & 463 & 477 & 487 & 490 \\
\hline $\mathrm{SiO}_{2}$ & 46.77 & 47.12 & 46.96 & 46.51 & 46.22 & 46.84 & 45.89 & 46.86 & 46.84 \\
\hline $\mathrm{Al}_{2} \mathrm{O}_{3}$ & 14.60 & 14.65 & 14.64 & 14.83 & 14.84 & 15.26 & 14.57 & 14.27 & 14.90 \\
\hline $\mathrm{TiO}_{2}$ & 2.51 & 2.54 & 2.55 & 2.40 & 2.45 & 1.68 & 2.03 & 3.15 & 2.88 \\
\hline $\mathrm{FeO}$ & 13.32 & 13.23 & 13.16 & 13.05 & 13.54 & 11.94 & 12.35 & 14.46 & 13.79 \\
\hline $\mathrm{MnO}$ & .20 & .20 & .20 & .19 & .19 & .18 & .19 & .21 & .20 \\
\hline $\mathrm{CaO}$ & 9.35 & 9.40 & 9.65 & 9.62 & 9.74 & 10.40 & 9.95 & 9.25 & 9.24 \\
\hline $\mathrm{MgO}$ & 7.48 & 7.70 & 7.67 & 8.12 & 7.59 & 9.08 & 9.07 & 6.32 & 6.76 \\
\hline $\mathrm{K}_{2} \mathrm{O}$ & .59 & .61 & .60 & .54 & .46 & .37 & .42 & .72 & .66 \\
\hline $\mathrm{Na}_{2} \mathrm{O}$ & 2.65 & 2.61 & 2.58 & 2.64 & 2.54 & 2.31 & 2.45 & 2.81 & 2.81 \\
\hline $\mathrm{P}_{2} \mathrm{O}_{5}$ & .58 & .56 & .54 & .47 & .49 & .28 & .40 & .54 & .50 \\
\hline Total & 98.05 & 98.62 & 98.55 & 98.37 & 98.06 & 98.34 & 97.32 & 98.59 & 98.58 \\
\hline Oxide & 521 & 545 & 576 & 592 & 630 & 659 & 673 & 730 & 752 \\
\hline $\mathrm{SiO}_{2}$ & 47.56 & 46.10 & 46.25 & 46.04 & 46.28 & 46.38 & 46.19 & 46.48 & 47.10 \\
\hline $\mathrm{Al}_{2} \mathrm{O}_{3}$ & 13.71 & 14.49 & 15.08 & 14.52 & 14.85 & 14.49 & 14.67 & 14.60 & 13.59 \\
\hline $\mathrm{TiO}_{2}$ & 3.53 & 3.09 & 2.86 & 3.05 & 2.88 & 3.15 & 2.87 & 2.96 & 3.10 \\
\hline $\mathrm{FeO}$ & 15.19 & 14.96 & 14.59 & 14.80 & 14.16 & 14.99 & 14.30 & 14.25 & 14.48 \\
\hline $\mathrm{MnO}$ & .22 & .21 & .20 & .21 & .20 & .21 & .21 & .20 & .22 \\
\hline $\mathrm{CaO}$ & 9.34 & 9.38 & 9.57 & 9.61 & 9.55 & 9.78 & 9.46 & 9.57 & 9.44 \\
\hline $\mathrm{MgO}$ & 6.05 & 6.20 & 7.01 & 7.01 & 7.40 & 6.76 & 6.99 & 7.10 & 7.24 \\
\hline $\mathrm{K}_{2} \mathrm{O}$ & .74 & .65 & .46 & .47 & .44 & .46 & .49 & .55 & .62 \\
\hline $\mathrm{Na}_{2} \mathrm{O}$ & 2.93 & 2.79 & 2.78 & 2.86 & 2.80 & 2.81 & 2.88 & 2.81 & 2.45 \\
\hline $\mathrm{P}_{2} \mathrm{O}_{5}$ & .58 & .51 & .42 & .48 & .43 & .47 & .46 & .45 & .72 \\
\hline Total & 99.85 & 98.38 & 99.22 & 99.05 & 98.99 & 99.50 & 98.52 & 98.97 & 98.96 \\
\hline Oxide & 785 & 816 & 832 & 870 & 886 & 923 & 961 & 990 & \\
\hline $\mathrm{SiO}_{2}$ & 46.20 & 46.22 & 47.97 & 47.29 & 46.89 & 46.56 & 47.09 & 46.42 & \\
\hline $\mathrm{Al}_{2} \mathrm{O}_{3}$ & 14.06 & 13.88 & 14.04 & 13.95 & 14.44 & 14.51 & 14.76 & 14.46 & \\
\hline $\mathrm{TiO}_{2}$ & 3.15 & 3.15 & 3.10 & 2.96 & 2.58 & 2.45 & 2.56 & 2.93 & \\
\hline $\mathrm{FeO}$ & 14.72 & 14.82 & 14.09 & 14.23 & 13.25 & 13.31 & 12.83 & 13.95 & \\
\hline $\mathrm{MnO}$ & .21 & .22 & .21 & .21 & .20 & .20 & .19 & .20 & \\
\hline $\mathrm{CaO}$ & 9.62 & 9.41 & 9.36 & 9.36 & 9.82 & 9.64 & 10.15 & 9.80 & \\
\hline $\mathrm{MgO}$ & 6.77 & 6.93 & 6.64 & 6.80 & 7.40 & 7.63 & 8.13 & 7.03 & \\
\hline $\mathrm{K}_{2} \mathrm{O}$ & .48 & .54 & .65 & .66 & .68 & .60 & .61 & .66 & \\
\hline $\mathrm{Na}_{2} \mathrm{O}$ & 2.61 & 2.52 & 2.59 & 2.49 & 2.52 & 2.61 & 2.53 & 2.64 & \\
\hline $\mathrm{P}_{2} \mathrm{O}_{5}$ & .58 & .59 & .55 & .53 & .55 & .53 & .60 & .56 & \\
\hline Total & 98.40 & 98.28 & 99.20 & 98.48 & 98.33 & 98.04 & 99.45 & 98.65 & \\
\hline
\end{tabular}




\section{B: TAN CH 1}

\begin{tabular}{|c|c|c|c|c|c|c|c|c|c|}
\hline \multirow[b]{2}{*}{ Oxide } & \multicolumn{9}{|c|}{ Depth } \\
\hline & 61 & 91 & 111 & 130 & 150 & 195 & 250 & 300 & 380 \\
\hline $\mathrm{SiO}_{2}$ & 46.29 & 45.76 & 46.06 & 45.51 & 46.78 & 48.18 & 47.54 & 46.77 & 46.60 \\
\hline $\mathrm{Al}_{2} \mathrm{O}_{3}$ & 13.06 & 14.15 & 14.27 & 13.90 & 13.81 & 14.58 & 14.96 & 14.54 & 14.82 \\
\hline $\mathrm{TiO}_{2}$ & 3.82 & 2.83 & 3.25 & 3.33 & 3.38 & 2.89 & 2.45 & 2.52 & 2.02 \\
\hline $\mathrm{FeO}$ & 15.44 & 13.93 & 14.73 & 14.92 & 14.90 & 13.42 & 13.20 & 13.08 & 12.07 \\
\hline $\mathrm{MnO}$ & .23 & .21 & .22 & 22 & .22 & .21 & .20 & .19 & .19 \\
\hline $\mathrm{CaO}$ & 10.18 & 9.50 & 9.72 & 9.63 & 9.59 & 9.41 & 9.40 & 9.41 & 10.10 \\
\hline $\mathrm{MgO}$ & 5.87 & 7.07 & 7.04 & 6.98 & 6.88 & 6.94 & 7.49 & 7.87 & 9.38 \\
\hline $\mathrm{K}_{2} \mathrm{O}$ & .64 & .58 & .56 & .58 & .68 & .71 & .56 & .57 & .43 \\
\hline $\mathrm{Na}_{2} \mathrm{O}$ & 2.61 & 2.58 & 2.57 & 2.55 & 2.58 & 2.68 & 2.70 & 2.59 & 2.48 \\
\hline $\mathrm{P}_{2} \mathrm{O}_{5}$ & 1.37 & .91 & .83 & .86 & .84 & .74 & .58 & .53 & .38 \\
\hline Total & 99.51 & 97.52 & 99.25 & 98.48 & 99.66 & 99.76 & 99.08 & 98.07 & 98.47 \\
\hline Oxide & 421 & 435 & 460 & 475 & 491 & 500 & 521 & 571 & 593 \\
\hline $\mathrm{SiO}_{2}$ & 47.23 & 46.39 & 46.88 & 46.37 & 45.91 & 46.62 & 46.23 & 46.29 & 45.95 \\
\hline $\mathrm{Al}_{2} \mathrm{O}_{3}$ & 15.25 & 15.09 & 14.79 & 14.84 & 14.29 & 14.52 & 14.86 & 14.85 & 14.66 \\
\hline $\mathrm{TiO}_{2}$ & 2.24 & 2.23 & 2.95 & 2.87 & 3.18 & 3.04 & 3.06 & 2.87 & 2.89 \\
\hline $\mathrm{FeO}$ & 13.06 & 12.77 & 13.99 & 14.04 & 14.73 & 14.27 & 14.06 & 14.56 & 14.31 \\
\hline $\mathrm{MnO}$ & .20 & .19 & .20 & .20 & .21 & .21 & .21 & .21 & .20 \\
\hline $\mathrm{CaO}$ & 10.48 & 10.46 & 9.31 & 9.24 & 9.39 & 9.35 & 9.67 & 9.54 & 9.52 \\
\hline $\mathrm{MgO}$ & 7.69 & 7.75 & 6.81 & 6.73 & 6.28 & 6.81 & 6.96 & 7.31 & 7.15 \\
\hline $\mathrm{K}_{2} \mathrm{O}$ & .37 & .34 & .62 & .60 & .59 & .61 & .45 & .49 & .49 \\
\hline $\mathrm{Na}_{2} \mathrm{O}$ & 2.55 & 2.50 & 2.76 & 2.80 & 2.67 & 2.74 & 2.75 & 2.78 & 2.69 \\
\hline $\mathrm{P}_{2} \mathrm{O}_{5}$ & .47 & .39 & .50 & .50 & .53 & .50 & .38 & .46 & .44 \\
\hline Total & 99.54 & 98.11 & 98.81 & 98.19 & 97.78 & 98.67 & 98.63 & 99.36 & 98.30 \\
\hline
\end{tabular}




\section{C: NPR}

[NPR Test]

\begin{tabular}{|c|c|c|c|c|c|c|c|c|c|}
\hline \multirow[b]{2}{*}{ Oxide } & \multicolumn{9}{|c|}{ Depth } \\
\hline & 36 & $36 \mathrm{R}$ & 56 & 98 & 121 & 127 & 141 & 156 & 170 \\
\hline $\mathrm{SiO}_{2}$ & 46.35 & 46.81 & 45.96 & 46.95 & 47.90 & 47.50 & 47.31 & 47.26 & 47.58 \\
\hline $\mathrm{Al}_{2} \mathrm{O}_{3}$ & 14.62 & 14.66 & 14.55 & 14.60 & 14.86 & 14.86 & 14.55 & 14.95 & 14.93 \\
\hline $\mathrm{TiO}_{2}$ & 2.90 & 2.97 & 2.84 & 2.66 & 2.07 & 2.26 & 3.05 & 2.43 & 2.40 \\
\hline $\mathrm{FeO}$ & 13.89 & 13.74 & 13.75 & 13.14 & 11.71 & 11.69 & 13.62 & 12.48 & 12.71 \\
\hline $\mathrm{MnO}$ & .20 & .20 & .20 & .20 & .18 & .19 & .21 & .19 & .19 \\
\hline $\mathrm{CaO}$ & 9.45 & 9.53 & 9.39 & 9.46 & 9.94 & 10.09 & 9.43 & 9.33 & 9.35 \\
\hline $\mathrm{MgO}$ & 7.32 & 7.37 & 7.45 & 7.63 & 8.96 & 8.54 & 6.98 & 7.51 & 7.88 \\
\hline $\mathrm{K}_{2} \mathrm{O}$ & .61 & .61 & .59 & .71 & .67 & .65 & .79 & .85 & .84 \\
\hline $\mathrm{Na}_{2} \mathrm{O}$ & 2.77 & 2.78 & 2.71 & 2.75 & 2.53 & 2.55 & 2.75 & 2.73 & 2.67 \\
\hline $\mathrm{P}_{2} \mathrm{O}_{5}$ & .66 & .67 & .64 & .61 & .46 & .51 & .66 & .63 & .62 \\
\hline Total & 98.77 & 99.34 & 98.08 & 98.71 & 99.28 & 98.84 & 99.35 & 98.36 & 99.17 \\
\hline Oxide & 232 & 273 & 291 & 311 & 379 & 450 & 461 & 480 & 489 \\
\hline $\mathrm{SiO}_{2}$ & 47.16 & 46.83 & 46.64 & 47.49 & 47.43 & 47.11 & 47.01 & 47.28 & 47.72 \\
\hline $\mathrm{Al}_{2} \mathrm{O}_{3}$ & 15.00 & 14.34 & 15.26 & 15.31 & 15.52 & 16.26 & 14.76 & 11.80 & 15.07 \\
\hline $\mathrm{TiO}_{2}$ & 2.64 & 3.25 & 2.86 & 1.95 & 1.69 & 1.32 & 2.77 & 4.59 & 2.68 \\
\hline $\mathrm{FeO}$ & 13.22 & 14.30 & 13.78 & 11.79 & 11.39 & 10.55 & 13.33 & 15.58 & 13.07 \\
\hline $\mathrm{MnO}$ & .20 & .21 & .20 & .19 & .19 & .18 & .20 & .23 & .20 \\
\hline $\mathrm{CaO}$ & 9.54 & 9.61 & 9.66 & 10.45 & 10.81 & 11.64 & 9.61 & 9.42 & 9.68 \\
\hline $\mathrm{MgO}$ & 7.76 & 6.61 & 7.65 & 9.02 & 9.18 & 10.12 & 7.46 & 4.69 & 7.11 \\
\hline $\mathrm{K}_{2} \mathrm{O}$ & .70 & .86 & .61 & .45 & .38 & .12 & .65 & 1.06 & .69 \\
\hline $\mathrm{Na}_{2} \mathrm{O}$ & 2.75 & 2.68 & 2.66 & 2.42 & 2.44 & 2.25 & 2.54 & 2.73 & 2.62 \\
\hline $\mathrm{P}_{2} \mathrm{O}_{5}$ & .69 & .74 & .63 & .43 & .35 & .21 & .59 & .92 & .58 \\
\hline Total & 99.66 & 99.43 & 99.95 & 99.50 & 99.38 & 99.76 & 98.92 & 98.30 & 99.42 \\
\hline Oxide & 527 & 538 & 550 & 608 & & & & & \\
\hline $\mathrm{SiO}_{2}$ & 47.64 & 47.74 & 47.86 & 46.67 & & & & & \\
\hline $\mathrm{Al}_{2} \mathrm{O}_{3}$ & 15.37 & 15.51 & 15.47 & 14.45 & & & & & \\
\hline $\mathrm{TiO}_{2}$ & 1.35 & 1.36 & 1.33 & 2.51 & & & & & \\
\hline $\mathrm{FeO}$ & 10.77 & 10.55 & 10.62 & 13.23 & & & & & \\
\hline $\mathrm{MnO}$ & .17 & .17 & .18 & .20 & & & & & \\
\hline $\mathrm{CaO}$ & 10.36 & 10.54 & 10.64 & 10.16 & & & & & \\
\hline $\mathrm{MgO}$ & 10.96 & 10.59 & 10.39 & 8.11 & & & & & \\
\hline $\mathrm{K}_{2} \mathrm{O}$ & .40 & .40 & .40 & .55 & & & & & \\
\hline $\mathrm{Na}_{2} \mathrm{O}$ & 2.26 & 2.22 & 2.25 & 2.56 & & & & & \\
\hline $\mathrm{P}_{2} \mathrm{O}_{5}$ & .23 & .23 & .22 & .50 & & & & & \\
\hline Total & 99.51 & 99.31 & 99.36 & 98.94 & & & & & \\
\hline \multicolumn{10}{|c|}{ [NPR WO1] } \\
\hline Oxide & 418 & 448 & $448 \mathrm{R}$ & 476 & 522 & & & & \\
\hline $\mathrm{SiO}_{2}$ & 47.43 & 47.53 & 47.78 & 47.02 & 47.06 & & & & \\
\hline $\mathrm{Al}_{2} \mathrm{O}_{3}$ & 15.87 & 16.27 & 16.31 & 14.54 & 15.63 & & & & \\
\hline $\mathrm{TiO}_{2}$ & 1.34 & 1.17 & 1.17 & 2.76 & 1.33 & & & & \\
\hline $\mathrm{FeO}$ & 10.66 & 10.16 & 10.14 & 13.72 & 10.81 & & & & \\
\hline $\mathrm{MnO}$ & .18 & .17 & .17 & .20 & .18 & & & · & \\
\hline $\mathrm{CaO}$ & 11.51 & 11.29 & 11.32 & 9.52 & 10.48 & & & & \\
\hline $\mathrm{MgO}$ & 9.48 & 10.46 & 10.52 & 7.42 & 11.08 & & & & \\
\hline $\mathrm{K}_{2} \mathrm{O}$ & .24 & .23 & .22 & .68 & .29 & & & & \\
\hline $\mathrm{Na}_{2} \mathrm{O}$ & 2.32 & 2.29 & 2.28 & 2.60 & 2.18 & & & & \\
\hline $\mathrm{P}_{2} \mathrm{O}_{5}$ & .22 & .19 & .19 & .59 & .23 & & & & \\
\hline Total & 99.25 & 99.76 & 100.10 & 99.05 & 99.27 & & & & \\
\hline
\end{tabular}




\section{D: TRA 5}

\begin{tabular}{lrrrrrr}
\hline & \multicolumn{7}{c}{ Depth } \\
\cline { 2 - 7 } Oxide & 100 & 139 & 162 & 170 & 192 & 202 \\
\hline $\mathrm{SiO}_{2}$ & 47.21 & 47.06 & 47.73 & 46.71 & 46.99 & 46.71 \\
$\mathrm{Al}_{2} \mathrm{O}_{3}$ & 15.34 & 15.11 & 15.10 & 15.06 & 14.25 & 13.67 \\
$\mathrm{TiO}_{2}$ & 2.10 & 2.13 & 2.19 & 1.95 & 2.88 & 3.00 \\
$\mathrm{FeO}$ & 12.21 & 12.23 & 12.66 & 12.26 & 13.68 & 14.06 \\
$\mathrm{MnO}$ & .19 & .19 & .19 & .19 & .21 & .21 \\
$\mathrm{CaO}$ & 9.87 & 9.79 & 10.04 & 9.67 & 10.23 & 9.63 \\
$\mathrm{MgO}$ & 9.18 & 9.16 & 8.07 & 8.92 & 7.42 & 7.51 \\
$\mathrm{~K}_{2} \mathrm{O}$ & .59 & .60 & .60 & .54 & .65 & .82 \\
$\mathrm{Na}_{2} \mathrm{O}$ & 2.54 & 2.56 & 2.59 & 2.58 & 2.60 & 2.64 \\
$\mathrm{P}_{2} \mathrm{O}_{5}$ & .44 & .41 & .42 & .39 & .75 & .79 \\
Total & 99.67 & 99.24 & 99.59 & 98.27 & 99.66 & 99.04 \\
\hline
\end{tabular}


Table 3.-Trace-element concentrations of selected core subsamples, by corehole and analytical method, Idaho National Engineering Laboratory, Idaho

[Location of coreholes is shown on figure 2. Units are parts per million (ppm) except for depth, which is feet below land surface. Abbreviations: TAN, Test Area North; $\mathrm{CH}$, corehole; NPR, New Production Reactor; TRA, Test Reactors Area; ICP/MS, inductively coupled plasma mass spectrometry; XRF, wavelength-dispersive X-ray fluorescence spectrometry. Analyses by Washington State University. Depth: R indicates quality-assurance split subsample of the specified sample depth. Quality-assurance samples were not included in calculation of statistical parameters. Symbol: ND indicates that analysis was not requested]

\section{TRACE ELEMENTS}

$\begin{array}{llll}\text { A: } & \text { Barium } & \text { Q: } & \text { Niobium } \\ \text { B: } & \text { Cerium } & \text { R: } & \text { Praseodymium } \\ \text { C: } & \text { Chromium } & \text { S: } & \text { Rubidium } \\ \text { D: } & \text { Copper } & \text { T: } & \text { Samarium } \\ \text { E: } & \text { Dysprosium } & \text { U: } & \text { Scandium } \\ \text { F: } & \text { Erbium } & \text { V: } & \text { Strontium } \\ \text { G: } & \text { Europium } & \text { W: } & \text { Tantalum } \\ \text { H: } & \text { Gadolinium } & \text { X: } & \text { Terbium } \\ \text { I: } & \text { Gallium } & \text { Y: } & \text { Thorium } \\ \text { J: } & \text { Hafnium } & \text { Z: } & \text { Thulium } \\ \text { K: } & \text { Holmium } & \text { AA: } & \text { Uranium } \\ \text { L: } & \text { Lanthanum } & \text { BB: } & \text { Vanadium } \\ \text { M: } & \text { Lead } & \text { CC: } & \text { Ytterbium } \\ \text { N: } & \text { Lutetium } & \text { DD: } & \text { Yttrium } \\ \text { O: } & \text { Neodymium } & \text { EE: } & \text { Zinc } \\ \text { P: } & \text { Nickel } & \text { FF: } & \text { Zirconium }\end{array}$


A: Barium (Ba)-Average crustal abundance is 390 ppm (Emsley, 1989)

\begin{tabular}{|c|c|c|c|c|c|c|c|c|c|c|}
\hline \multicolumn{11}{|c|}{ [Concentration] } \\
\hline \multicolumn{3}{|c|}{ TAN CH 2} & \multicolumn{3}{|c|}{ TAN CH 1} & \multicolumn{3}{|c|}{ NPR Test } & \multicolumn{2}{|c|}{ NPR W01 } \\
\hline Depth & ICP/MS & XRF & Depth & ICP/MS & $\overline{\text { XRF }}$ & Depth & ICP/MS & $\overline{\text { XRF }}$ & Depth & $\overline{\mathrm{XAF}}$ \\
\hline 138 & 483 & 452 & 61 & 595 & 553 & 36 & ND & 314 & 418 & 110 \\
\hline 154 & 458 & 424 & 91 & 469 & 421 & $36 \mathrm{R}$ & ND & 331 & 448 & 89 \\
\hline 172 & 458 & 423 & 111 & 419 & 380 & 56 & ND & 304 & $448 R$ & 88 \\
\hline 201 & 442 & 407 & 130 & 437 & 421 & 98 & ND & 363 & 476 & 411 \\
\hline 215 & 473 & 434 & 150 & 461 & 441 & 121 & ND & 244 & 522 & 215 \\
\hline 238 & 448 & 422 & 195 & 470 & 424 & 127 & ND & 277 & & \\
\hline 256 & 487 & 461 & $195 \mathrm{R}$ & 464 & ND & 141 & ND & 398 & & \\
\hline 277 & 394 & 368 & 250 & 398 & 362 & 156 & ND & 446 & \multicolumn{2}{|c|}{ TRA 5} \\
\hline 299 & 450 & 416 & 300 & 359 & 333 & 170 & ND & 421 & Depth & XRF \\
\hline 309 & 360 & 345 & 380 & 254 & 218 & 232 & ND & 406 & 100 & 277 \\
\hline 327 & 370 & 345 & 421 & 319 & 273 & 273 & ND & 488 & 139 & 277 \\
\hline 352 & 392 & 330 & 435 & 272 & 233 & 291 & 374 & 345 & 162 & 257 \\
\hline 366 & 344 & 313 & 460 & 395 & 379 & 311 & ND & 239 & 170 & 232 \\
\hline 413 & 402 & 374 & 475 & 396 & 378 & 379 & ND & 197 & 192 & 454 \\
\hline 463 & 224 & 197 & 491 & 446 & 432 & 450 & 170 & 146 & 202 & 440 \\
\hline 477 & 265 & 226 & 500 & 387 & 357 & 461 & ND & 415 & & \\
\hline 487 & 451 & 424 & 521 & 306 & 276 & 480 & ND & 620 & & \\
\hline 490 & 484 & 445 & 571 & 311 & 290 & 489 & ND & 420 & & \\
\hline 521 & 481 & 454 & 593 & 308 & 287 & 527 & 202 & 162 & & \\
\hline 545 & 466 & 436 & 593R & 308 & ND & 538 & ND & 174 & & \\
\hline 576 & 339 & 314 & & & & 550 & ND & 167 & & \\
\hline 592 & 339 & 293 & & & & 608 & ND & 257 & & \\
\hline 630 & 311 & 263 & & & & & & & & \\
\hline 659 & 337 & 330 & & & & & & & & \\
\hline 673 & 357 & 310 & & & & & & & & \\
\hline 730 & 327 & 293 & & & & & & & & \\
\hline 752 & 383 & 366 & & & & & & & & \\
\hline 785 & 369 & 344 & & & & & & & & \\
\hline 816 & 374 & 370 & & & & & & & & \\
\hline 832 & 412 & 377 & & & & & & & & \\
\hline 870 & 384 & 370 & & & & & & & & \\
\hline 886 & 390 & 346 & & & & & & & & \\
\hline 923 & 394 & 367 & & & & & & & & \\
\hline $923 \mathrm{R}$ & 397 & ND & & & & & & & & \\
\hline 961 & 377 & 349 & & & & & & & & \\
\hline 990 & 414 & 370 & & & & & & & & \\
\hline
\end{tabular}

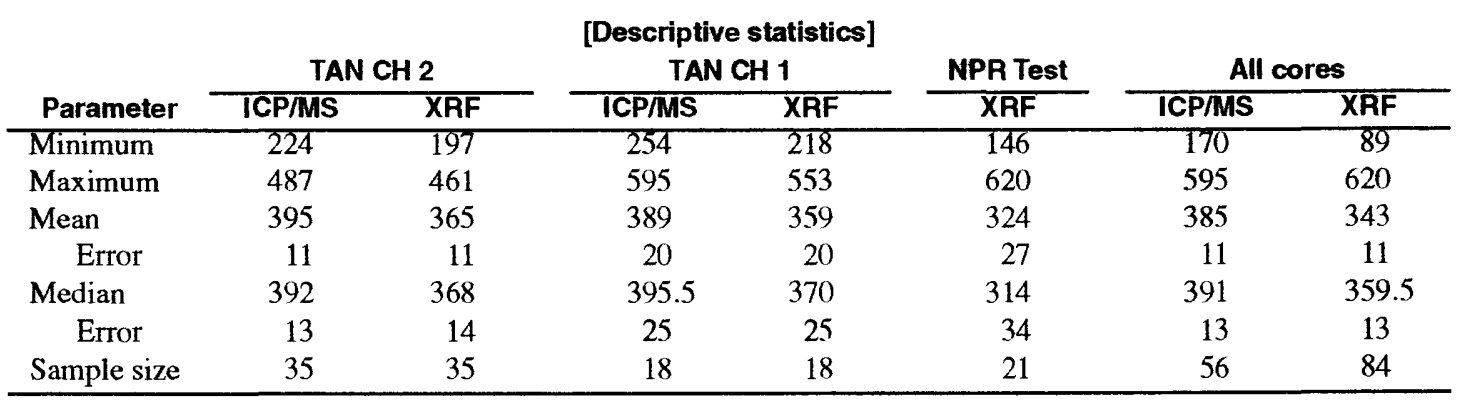


B: Cerium (Ce)-Average crustal abundance is $66 \mathrm{ppm}$ (Emsley, 1989)

[Ce concentrations by XRF are qualitative estimates]

\begin{tabular}{|c|c|c|c|c|c|c|c|c|c|c|c|}
\hline \multicolumn{12}{|c|}{ [Concentration] } \\
\hline \multicolumn{3}{|c|}{ TAN CH 2} & \multicolumn{3}{|c|}{ TAN CH 1} & \multicolumn{3}{|c|}{ NPR Test } & \multicolumn{3}{|c|}{ NPR W01 } \\
\hline Depth & ICP/MS & XRF & Depth & ICP/MS & XRF & Depth & ICP/MS & XRF & Depth & & \\
\hline 138 & 102 & 105 & 61 & 140) & 146 & 36 & ND & 61 & 418 & & \\
\hline 154 & 97 & 99 & 91 & 93 & 90 & $36 \mathrm{R}$ & ND & 55 & 448 & & \\
\hline 172 & 82 & 84 & 111 & 78 & 82 & 56 & ND & 64 & $448 \mathrm{R}$ & & 7 \\
\hline 201 & 78 & 78 & 130 & 83 & 98 & 98 & ND & 68 & 476 & & 3 \\
\hline 215 & 83 & 85 & 150 & 83 & 88 & 121 & ND & 43 & 522 & & 2 \\
\hline 238 & 78 & 94 & 195 & 84 & 76 & 127 & ND & 46 & & & \\
\hline 256 & 84 & 88 & $195 \mathrm{R}$ & 82 & ND & 141 & ND & 51 & \multirow{2}{*}{\multicolumn{3}{|c|}{ TRA 5}} \\
\hline 277 & 66 & 80 & 250 & 63 & 38 & 156 & ND & 66 & & & \\
\hline 299 & 82 & 76 & 300 & 55 & 61 & 170 & ND & 77 & Depth & & $\overline{F F}$ \\
\hline 309 & 65 & 70 & 380 & 38 & 34 & 232 & ND & 79 & 100 & & \\
\hline 327 & 59 & 61 & 421 & 48 & 44 & 273 & ND & 66 & 139 & & 3 \\
\hline 352 & 57 & 72 & 435 & 40 & 45 & 291 & 56 & 54 & 162 & & 5 \\
\hline 366 & 51 & 39 & 460 & 49 & 57 & 311 & ND & 30 & 170 & & 4 \\
\hline 413 & 50 & 52 & 475 & 50 & 54 & 379 & ND & 30 & 192 & & \\
\hline 463 & 29 & 32 & 491 & 51 & 60 & 450 & 21 & 23 & 202 & & \\
\hline 477 & 39 & 34 & 500 & 48 & 54 & 461 & ND & 43 & & & \\
\hline 487 & 54 & 38 & 521 & 37 & 44 & 480 & ND & 94 & & & \\
\hline 490 & 52 & 47 & 571 & 39 & 43 & 489 & ND & 61 & & & \\
\hline 521 & 59 & 43 & 593 & 40 & 33 & 527 & 26 & 26 & & & \\
\hline 545 & 50 & 44 & 593R & 39 & ND & 538 & ND & 26 & & & \\
\hline 576 & 38 & 44 & & & & 550 & ND & 24 & & & \\
\hline 592 & 42 & 46 & & & & 608 & ND & 55 & & & \\
\hline 630 & 38 & 46 & & & & & & & & & \\
\hline 659 & 42 & 55 & & & & & & & & & \\
\hline 673 & 44 & 53 & & & & & & & & & \\
\hline 730 & 41 & 48 & & & & & & & & & \\
\hline 752 & 64 & 62 & & & & & & & & & \\
\hline 785 & 51 & 58 & & & & & & & & & \\
\hline 816 & 53 & 51 & & & & & & & & & \\
\hline 832 & 52 & 62 & & & & & & & & & \\
\hline 870 & 50 & 75 & & & & & & & & & \\
\hline 886 & 52 & 35 & & & & & & & & & \\
\hline 923 & 50 & 49 & & & & & & & & & \\
\hline $923 \mathrm{R}$ & 49 & ND & & & & & & & & & \\
\hline 961 & 54 & 48 & & & & & & & & & \\
\hline \multirow[t]{2}{*}{990} & 51 & 46 & & & & & & & & & \\
\hline & \multicolumn{11}{|c|}{ [Descriptive statistics] } \\
\hline & arameter & ICP/MS & XRF & $\overline{I C P}$ & & $\overline{\mathrm{XRF}}$ & XRF & TCP/MS & $\bar{x}$ & & \\
\hline & inimum & 29 & 32 & & & 33 & 23 & 21 & & & \\
\hline & aximum & 102 & 105 & 14 & & 146 & 94 & 140 & $1<$ & & \\
\hline & ean & 58 & 60 & & & 64 & 52 & 58 & & & \\
\hline & Error & 3 & 3.3 & & 5.3 & 6.8 & 4.4 & 2.9 & & 2.5 & \\
\hline & edian & 52 & 53 & & .5 & 55.5 & 54 & 52 & & .5 & \\
\hline & Error & 3.7 & 4.2 & & 8.9 & 8.5 & 5.5 & 3.6 & & 3.1 & \\
\hline & Imple size & 35 & 35 & & & 18 & 21 & 56 & & & \\
\hline
\end{tabular}


C: Chromium (Cr) - Average crustal abundance is 122 ppm (Emsley, 1989)

\begin{tabular}{|c|c|c|c|c|c|c|c|}
\hline \multicolumn{8}{|c|}{ [Concentration] } \\
\hline \multicolumn{2}{|c|}{ TAN CH 2} & \multicolumn{2}{|c|}{ TAN CH 1} & \multicolumn{2}{|c|}{ NPR Test } & \multicolumn{2}{|c|}{ NPR WO1 } \\
\hline Depth & XRF & Depth & XRF & Depth & $\overline{X R F}$ & Depth & $\overline{X R F}$ \\
\hline 138 & 176 & 61 & 118 & 36 & 165 & 418 & 341 \\
\hline 154 & 182 & 91 & 185 & $36 \mathrm{R}$ & 167 & 448 & 374 \\
\hline 172 & 180 & 111 & 163 & 56 & 167 & $448 \mathrm{R}$ & 387 \\
\hline 201 & 197 & 130 & 169 & 98 & 213 & 476 & 186 \\
\hline 215 & 196 & 150 & 179 & 121 & 361 & 522 & 419 \\
\hline 238 & 205 & 195 & 178 & 127 & 318 & & \\
\hline 256 & 137 & 250 & 212 & 141 & 155 & & \\
\hline 277 & 208 & 300 & 223 & 156 & 213 & \multicolumn{2}{|c|}{ TRA 5} \\
\hline 299 & 156 & 380 & 380 & 170 & 235 & Depth & XRF \\
\hline 309 & 214 & 421 & 211 & 232 & 203 & 100 & 269 \\
\hline 327 & 210 & 435 & 213 & 273 & 127 & 139 & 268 \\
\hline 352 & 232 & 460 & 139 & 291 & 161 & 162 & 224 \\
\hline 366 & 247 & 475 & 143 & 311 & 303 & 170 & 232 \\
\hline 413 & 256 & 491 & 134 & 379 & 318 & 192 & 249 \\
\hline 463 & 297 & 500 & 145 & 450 & 380 & 202 & 239 \\
\hline 477 & 363 & 521 & 124 & 461 & 189 & & \\
\hline 487 & 131 & 571 & 177 & 480 & 61 & & \\
\hline 490 & 146 & 593 & 173 & 489 & 180 & & \\
\hline 521 & 107 & & & 527 & 406 & & \\
\hline 545 & 135 & & & 538 & 397 & & \\
\hline 576 & 132 & & & 550 & 393 & & \\
\hline 592 & 170 & & & 608 & 259 & & \\
\hline 630 & 186 & & & & & & \\
\hline 659 & 160 & & & & & & \\
\hline 673 & 168 & & & & & & \\
\hline 730 & 176 & & & & & & \\
\hline 752 & 183 & & & & & & \\
\hline 785 & 157 & & & & & & \\
\hline 816 & 159 & & & & & & \\
\hline 832 & 163 & & & & & & \\
\hline 870 & 182 & & & & & & \\
\hline 886 & 244 & & & & & & \\
\hline 923 & 267 & & & & & & \\
\hline 961 & 264 & & & & & & \\
\hline 990 & 173 & & & & & & \\
\hline
\end{tabular}

[Descriptive statistics]

\begin{tabular}{|c|c|c|c|c|}
\hline & TAN CH 2 & TAN CH 1 & NPR Test & All cores \\
\hline Parameter & XRF & XRF & XRF & XRF \\
\hline Minimum & 107 & 118 & 61 & 61 \\
\hline Maximum & 363 & 380 & 406 & 419 \\
\hline Mean & 193 & 181 & 248 & 215 \\
\hline Error & 8.9 & 14 & 22 & 8.5 \\
\hline Median & 182 & 175 & 213 & 187.5 \\
\hline Error & 11 & 17 & 28 & 11 \\
\hline Sample size & 35 & 18 & 21 & 84 \\
\hline
\end{tabular}


D: Copper $(\mathrm{Cu})$-Average crustal abundance is $68 \mathrm{ppm}$ (Emsley, 1989)

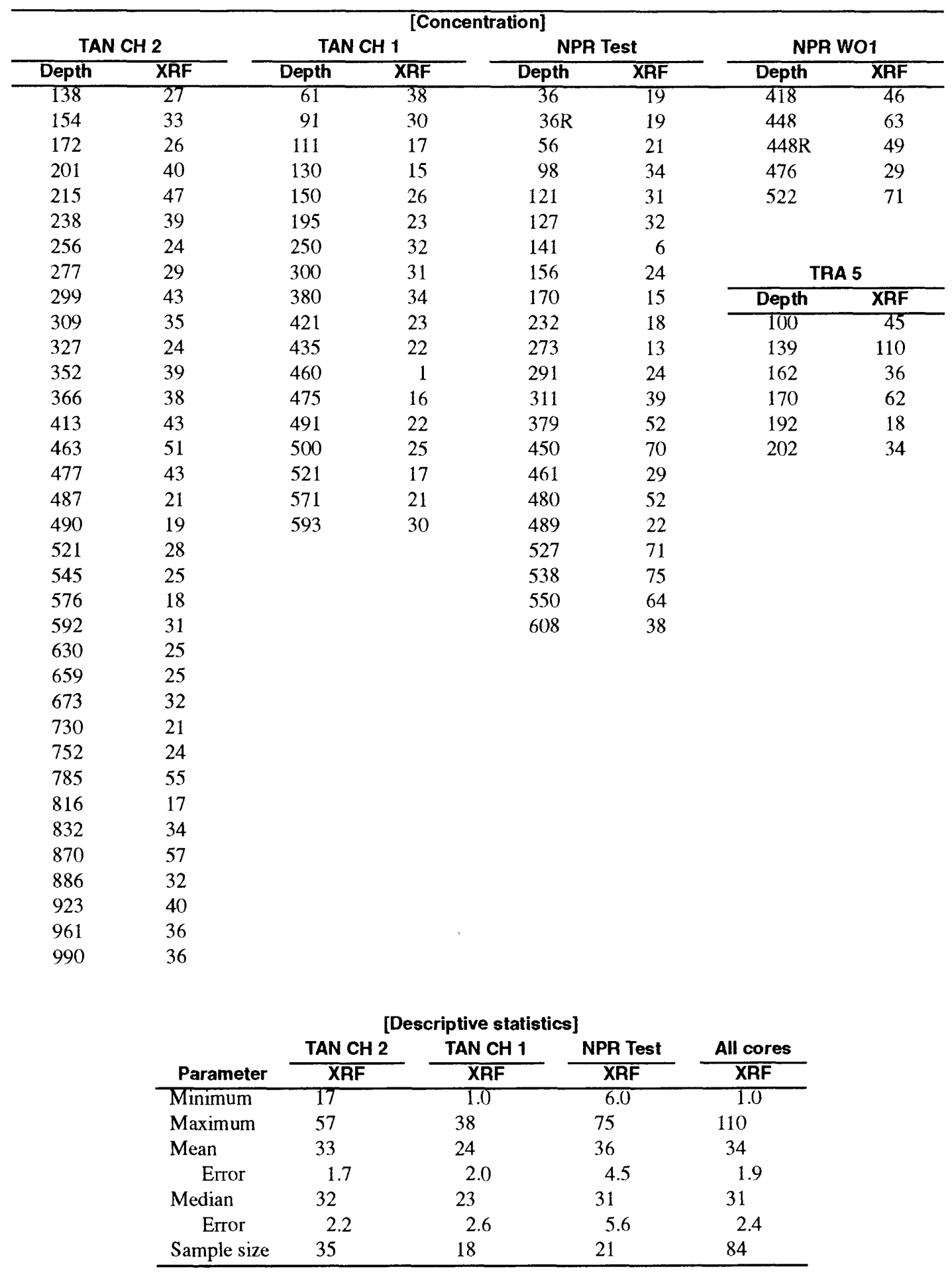


E: Dysprosium (Dy)—Average crustal abundance is 4.5 ppm (Emsley, 1989)

\begin{tabular}{|c|c|c|c|c|c|}
\hline \multicolumn{2}{|c|}{ TAN CH 2} & \multicolumn{2}{|c|}{ TAN CH 1} & NPR Test & est \\
\hline Depth & ICP/MS & Depth & ICP/MS & Depth & ICP/MS \\
\hline 138 & 9.6 & 61 & 12 & 291 & 6.1 \\
\hline 154 & 9.2 & 91 & 8.8 & 450 & 4.1 \\
\hline 172 & 8.5 & 111 & 8.3 & 527 & 4.1 \\
\hline 201 & 8.3 & 130 & 8.7 & & \\
\hline 215 & 8.8 & 150 & 9.2 & & \\
\hline 238 & 8.4 & 195 & 8.1 & & \\
\hline 256 & 7.5 & $195 \mathrm{R}$ & 7.8 & & \\
\hline 277 & 7.0 & 250 & 6.8 & & \\
\hline 299 & 8.6 & 300 & 6.4 & & \\
\hline 309 & 6.8 & 380 & 5.4 & & \\
\hline 327 & 6.7 & 421 & 6.2 & & \\
\hline 352 & 6.7 & 435 & 5.3 & & \\
\hline 366 & 6.0 & 460 & 6.2 & & \\
\hline 413 & 6.2 & 475 & 6.4 & & \\
\hline 463 & 4.5 & 491 & 6.9 & & \\
\hline 477 & 5.6 & 500 & 6.3 & & \\
\hline 487 & 6.6 & 521 & 6.0 & & \\
\hline 490 & 6.3 & 571 & 6.3 & & \\
\hline 521 & 7.4 & 593 & 6.1 & & \\
\hline 545 & 6.5 & $593 R$ & 6.2 & & \\
\hline 576 & 5.9 & & & & \\
\hline 592 & 6.3 & & & & \\
\hline 630 & 6.0 & & & & \\
\hline 659 & 6.5 & & & & \\
\hline 673 & 6.4 & & & & \\
\hline 730 & 6.1 & & & & \\
\hline 752 & 7.9 & & & & \\
\hline 785 & 7.2 & & & & \\
\hline 816 & 7.5 & & & & \\
\hline 832 & 7.4 & & & & \\
\hline 870 & 7.1 & & & & \\
\hline 886 & 7.1 & & & & \\
\hline 923 & 6.6 & & & & \\
\hline $923 R$ & 6.6 & & & & \\
\hline 961 & 7.4 & & & & \\
\hline 990 & 6.9 & & & & \\
\hline \multicolumn{6}{|c|}{ [Descriptive statistics] } \\
\hline & & TAN CH 2 & TAN CH 1 & All cores & \\
\hline & Parameter & ICP/MS & ICP/MS & ICP/MS & \\
\hline & Minimum & 4.5 & 5.3 & 4.1 & \\
\hline & Maximum & 9.6 & 12 & 12 & \\
\hline & Mean & 6.9 & 7.2 & 6.9 & \\
\hline & Error & .18 & .40 & .18 & \\
\hline & Median & 6.7 & 6.4 & 6.6 & \\
\hline & Error & .22 & .50 & .23 & \\
\hline & Sample size & 35 & 18 & 56 & \\
\hline
\end{tabular}


F: Erbium (Er) -Average crustal abundance is $3.5 \mathrm{ppm}$ (Emsley, 1989)

\begin{tabular}{|c|c|c|c|c|c|}
\hline \multicolumn{2}{|c|}{ TAN CH 2} & \multicolumn{2}{|c|}{$\begin{array}{c}\text { [Concentration] } \\
\text { TAN CH 1 }\end{array}$} & \multicolumn{2}{|c|}{ NPR Test } \\
\hline Depth & ICP/MS & Depth & ICP/MS & Depth & ICP/MS \\
\hline 138 & 5.1 & 61 & 6.4 & 291 & 3.3 \\
\hline 154 & 4.9 & 91 & 4.7 & 450 & 2.5 \\
\hline 172 & 4.6 & 111 & 4.4 & 527 & 2.5 \\
\hline 201 & 4.4 & 130 & 4.7 & & \\
\hline 215 & 4.7 & 150 & 4.9 & & \\
\hline 238 & 4.5 & 195 & 4.4 & & \\
\hline 256 & 4.0 & $195 R$ & 4.2 & & \\
\hline 277 & 3.6 & 250 & 3.7 & & \\
\hline 299 & 4.6 & 300 & 3.5 & & \\
\hline 309 & 3.6 & 380 & 3.1 & & \\
\hline 327 & 3.6 & 421 & 3.4 & & \\
\hline 352 & 3.6 & 435 & 2.9 & & \\
\hline 366 & 3.2 & 460 & 3.5 & & \\
\hline 413 & 3.4 & 475 & 3.5 & & \\
\hline 463 & 2.5 & 491 & 3.8 & & \\
\hline 477 & 3.1 & 500 & 3.4 & & \\
\hline 487 & 3.4 & 521 & 3.3 & & \\
\hline 490 & 3.4 & 571 & 3.4 & & \\
\hline 521 & 4.0 & 593 & 3.4 & & \\
\hline 545 & 3.5 & 593R & 3.4 & & \\
\hline 576 & 3.3 & & & & \\
\hline 592 & 3.5 & & & & \\
\hline 630 & 3.3 & & & & \\
\hline 659 & 3.6 & & & & \\
\hline 673 & 3.7 & & & & \\
\hline 730 & 3.4 & & & & \\
\hline 752 & 4.3 & & & & \\
\hline 785 & 3.8 & & & & \\
\hline 816 & 4.0 & & & & \\
\hline 832 & 4.0 & & & & \\
\hline 870 & 3.8 & & & & \\
\hline 886 & 3.8 & & & & \\
\hline 923 & 3.6 & & & & \\
\hline $923 \mathrm{R}$ & 3.7 & & & & \\
\hline 961 & 4.1 & & & & \\
\hline 990 & 3.8 & & & & \\
\hline \multicolumn{6}{|c|}{ [Descriptive statistics] } \\
\hline & & TAN CH 2 & TAN CH 1 & All core & \\
\hline & Parameter & ICP/MS & ICP/MS & ICP/MS & \\
\hline & Minimum & 2.5 & 2.9 & 2.5 & \\
\hline & Maximum & 5.1 & 6.4 & 6.4 & \\
\hline & Mean & 3.8 & 3.9 & 3.8 & \\
\hline & Error & .09 & .20 & .09 & \\
\hline & Median & 3.7 & 3.5 & 3.6 & \\
\hline & Error & .12 & .25 & .12 & \\
\hline & Sample size & 35 & 18 & 56 & \\
\hline
\end{tabular}


G: Europium (Eu)—Average crustal abundance is 2.1 ppm (Emsley, 1989)

\begin{tabular}{|c|c|c|c|c|c|}
\hline \multicolumn{6}{|c|}{ [Concentration] } \\
\hline \multicolumn{2}{|c|}{ TAN CH 2} & \multicolumn{2}{|c|}{ TAN CH 1} & \multicolumn{2}{|c|}{ NPR Test } \\
\hline Depth & $\mathrm{ICP} / \mathrm{MS}$ & Depth & ICP/MS & Depth & ICP/MS \\
\hline 138 & 3.6 & 61 & 4.5 & 291 & 2.4 \\
\hline 154 & 3.4 & 91 & 3.4 & 450 & 1.3 \\
\hline 172 & 3.3 & 111 & 3.3 & 527 & 1.3 \\
\hline 201 & 3.2 & 130 & 3.4 & & \\
\hline 215 & 3.3 & 150 & 3.5 & & \\
\hline 238 & 3.2 & 195 & 3.1 & & \\
\hline 256 & 2.9 & $195 \mathrm{R}$ & 3.0 & & \\
\hline 277 & 2.6 & 250 & 2.6 & & \\
\hline 299 & 3.1 & 300 & 2.4 & & \\
\hline 309 & 2.6 & 380 & 1.9 & & \\
\hline 327 & 2.6 & 421 & 2.3 & & \\
\hline 352 & 2.5 & 435 & 2.0 & & \\
\hline 366 & 2.2 & 460 & 2.5 & & \\
\hline 413 & 2.3 & 475 & 2.5 & & \\
\hline 463 & 1.5 & 491 & 2.7 & & \\
\hline 477 & 1.9 & 500 & 2.5 & & \\
\hline 487 & 2.7 & 521 & 2.2 & & \\
\hline 490 & 2.7 & 571 & 2.3 & & \\
\hline 521 & 2.9 & 593 & 2.3 & & \\
\hline 545 & 2.7 & $593 R$ & 2.3 & & \\
\hline 576 & 2.3 & & & & \\
\hline 592 & 2.4 & & & & \\
\hline 630 & 2.3 & & & & \\
\hline 659 & 2.5 & & & & \\
\hline 673 & 2.4 & & & & \\
\hline 730 & 2.4 & & & & \\
\hline 752 & 3.2 & & & & \\
\hline 785 & 2.9 & & & & \\
\hline 816 & 3.0 & & & & \\
\hline 832 & 2.9 & & & & \\
\hline 870 & 2.8 & & & & \\
\hline 886 & 2.7 & & & & \\
\hline 923 & 2.5 & & & & \\
\hline $923 R$ & 2.5 & & & & \\
\hline 961 & 2.7 & & & & \\
\hline 990 & 2.6 & & & & \\
\hline \multicolumn{6}{|c|}{ [Descriptive statistics] } \\
\hline & & TAN CH 2 & TAN CH 1 & All c & \\
\hline & Parameter & ICP/MS & ICP/MS & ICP/ & \\
\hline & Minimum & 1.5 & 1.9 & $\overline{1.2}$ & \\
\hline & Maximum & 3.6 & 4.5 & 4. & \\
\hline & Mean & 2.7 & 2.7 & 2. & \\
\hline & Error & .07 & .16 & & \\
\hline & Median & 2.7 & 2.5 & 2. & \\
\hline & Error & .09 & .20 & & \\
\hline & Sample size & 35 & 18 & 56 & \\
\hline
\end{tabular}


H: Gadolinium (Gd)—Average crustal abundance is $6.1 \mathrm{ppm}$ (Emsley, 1989)

\begin{tabular}{|c|c|c|c|c|c|}
\hline & & \multicolumn{2}{|c|}{$\begin{array}{c}\text { [Concentration] } \\
\text { TAN CH } 1\end{array}$} & \multicolumn{2}{|c|}{ NPR Test } \\
\hline Depth & ICP/MS & Depth & ICP/MS & Depth & ICP/MS \\
\hline 138 & 11 & 61 & 14 & 291 & 6.6 \\
\hline 154 & 11 & 91 & 10 & 450 & 3.8 \\
\hline 172 & 9.8 & 111 & 9.6 & 527 & 3.9 \\
\hline 201 & 9.2 & 130 & 9.9 & & \\
\hline 215 & 9.8 & 150 & 11 & & \\
\hline 238 & 9.2 & 195 & 9.2 & & \\
\hline 256 & 8.4 & $195 R$ & 8.4 & & \\
\hline 277 & 7.6 & 250 & 7.6 & & \\
\hline 299 & 9.6 & 300 & 7.1 & & \\
\hline 309 & 7.6 & 380 & 5.6 & & \\
\hline 327 & 7.4 & 421 & 6.6 & & \\
\hline 352 & 7.2 & 435 & 5.5 & & \\
\hline 366 & 6.6 & 460 & 6.9 & & \\
\hline 413 & 6.7 & 475 & 7.2 & & \\
\hline 463 & 4.6 & 491 & 7.6 & & \\
\hline 477 & 5.8 & 500 & 6.9 & & \\
\hline 487 & 7.4 & 521 & 6.4 & & \\
\hline 490 & 7.0 & 571 & 6.6 & & \\
\hline 521 & 8.0 & 593 & 6.5 & & \\
\hline 545 & 7.2 & 593R & 6.2 & & \\
\hline 576 & 6.3 & & & & \\
\hline 592 & 6.6 & & & & \\
\hline 630 & 6.1 & & & & \\
\hline 659 & 6.7 & & & & \\
\hline 673 & 7.0 & & & & \\
\hline 730 & 6.4 & & & & \\
\hline 752 & 8.9 & & & & \\
\hline 785 & 7.8 & & & & \\
\hline 816 & 8.1 & & & & \\
\hline 832 & 7.8 & & & & \\
\hline 870 & 7.6 & & & & \\
\hline 886 & 7.7 & & & & \\
\hline 923 & 7.3 & & & & \\
\hline $923 R$ & 7.0 & & & & \\
\hline 961 & 7.8 & & & & \\
\hline 990 & 7.6 & & & & \\
\hline
\end{tabular}

[Descriptive statistics]

\begin{tabular}{cccccc} 
& TAN CH 2 & & TAN CH 1 & & All cores \\
\cline { 2 - 3 } \cline { 5 - 6 } Parameter & ICP/MS & & ICP/MS & & ICP/MS \\
\hline Minimum & 4.6 & & 5.5 & & 3.8 \\
Maximum & 11 & & 14 & & 14 \\
Mean & 7.7 & & 8.0 & & 7.7 \\
$\quad$ Error & .24 & & .52 & & .24 \\
Median & 7.6 & & 7.15 & & 7.4 \\
$\quad$ Error & .30 & & .65 & & .30 \\
Sample size & 35 & & 18 & & 56 \\
\hline
\end{tabular}


I: Gallium (Ga) - Average crustal abundance is 19 ppm (Emsley, 1989)

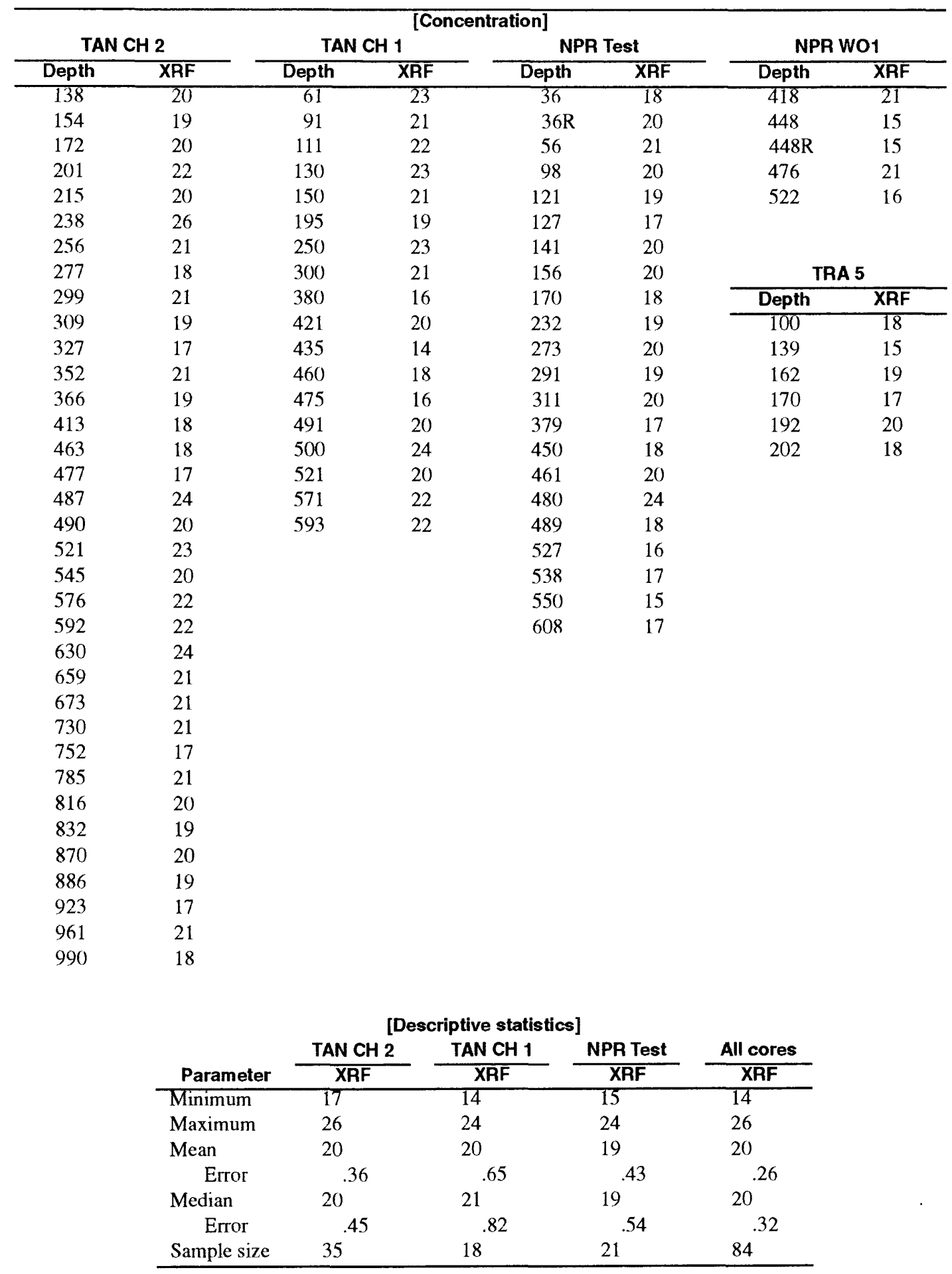


$\mathrm{J}$ : Hafnium (Hf)-Average crustal abundance is $2.8 \mathrm{ppm}$ (Emsley, 1989)

\begin{tabular}{|c|c|c|c|c|c|}
\hline \multicolumn{2}{|c|}{ TAN CH 2} & \multicolumn{2}{|c|}{ TAN CH 1} & \multicolumn{2}{|c|}{ NPR Test } \\
\hline Depth & ICP/MS & Depth & ICP/MS & Depth & ICP/MS \\
\hline 138 & 7.6 & 61 & 9.0 & 291 & 5.0 \\
\hline 154 & 6.2 & 91 & 6.0 & 450 & 2.5 \\
\hline 172 & 6.8 & 111 & 6.9 & 527 & 2.7 \\
\hline 201 & 6.8 & 130 & 7.4 & & \\
\hline 215 & 7.3 & 150 & 7.8 & & \\
\hline 238 & 6.5 & 195 & 6.6 & & \\
\hline 256 & 6.1 & $195 \mathrm{R}$ & 6.4 & & \\
\hline 277 & 5.3 & 250 & 5.3 & & \\
\hline 299 & 6.5 & 300 & 5.3 & & \\
\hline 309 & 5.3 & 380 & 4.0 & & \\
\hline 327 & 5.0 & 421 & 4.6 & & \\
\hline 352 & 5.4 & 435 & 3.9 & & \\
\hline 366 & 4.8 & 460 & 5.1 & & \\
\hline 413 & 4.8 & 475 & 5.2 & & \\
\hline 463 & 3.1 & 491 & 5.5 & & \\
\hline 477 & 4.0 & 500 & 5.0 & & \\
\hline 487 & 5.3 & 521 & 3.5 & & \\
\hline 490 & 4.9 & 571 & 4.7 & & \\
\hline 521 & 6.1 & 593 & 4.6 & & \\
\hline 545 & 5.2 & $593 R$ & 4.5 & & \\
\hline 576 & 4.3 & & & & \\
\hline 592 & 4.6 & & & & \\
\hline 630 & 4.4 & & & & \\
\hline 659 & 4.9 & & & & \\
\hline 673 & 5.0 & & & & \\
\hline 730 & 4.7 & & & & \\
\hline 752 & 6.7 & & & & \\
\hline 785 & 5.6 & & & & \\
\hline 816 & 6.1 & & & & \\
\hline 832 & 6.2 & & & & \\
\hline 870 & 5.8 & & & & \\
\hline 886 & 6.1 & & & & \\
\hline 923 & 5.9 & & & & \\
\hline $923 R$ & 5.8 & & & & \\
\hline 961 & 6.6 & & & & \\
\hline 990 & 5.3 & & & & \\
\hline
\end{tabular}

\begin{tabular}{|c|c|c|c|}
\hline & \multicolumn{2}{|c|}{ [Descriptive statistics] } & \multirow[b]{2}{*}{ All cores } \\
\hline & TAN CH 2 & TAN CH 1 & \\
\hline Parameter & ICP/MS & ICP/MS & ICP/MS \\
\hline Minimum & 3.1 & 3.5 & 2.5 \\
\hline Maximum & 7.6 & 9.0 & 9.0 \\
\hline Mean & 5.6 & 5.6 & 5.5 \\
\hline Error & .17 & .34 & .17 \\
\hline Median & 5.4 & 5.25 & 5.3 \\
\hline Error & .21 & .43 & .21 \\
\hline Sample size & 35 & 18 & 56 \\
\hline
\end{tabular}


K: Holmium (Ho)_Average crustal abundance is $1.3 \mathrm{ppm}$ (Emsley, 1989)

\begin{tabular}{|c|c|c|c|c|c|}
\hline \multicolumn{6}{|c|}{ [Concentration] } \\
\hline \multicolumn{2}{|c|}{ TAN CH 2} & \multicolumn{2}{|c|}{ TAN CH 1} & \multicolumn{2}{|c|}{ NPR Test } \\
\hline Depth & TCP/MS & Depth & ICP/MS & Depth & ICP/MS \\
\hline 138 & 1.8 & 61 & 2.2 & 291 & 1.2 \\
\hline 154 & 1.7 & 91 & 1.7 & 45() & .83 \\
\hline 172 & 1.6 & 111 & 1.6 & 527 & .84 \\
\hline 201 & 1.6 & 130 & 1.7 & & \\
\hline 215 & 1.6 & 150 & 1.7 & & \\
\hline 238 & 1.6 & 195 & 1.5 & & \\
\hline 256 & 1.4 & 195R & 1.4 & & \\
\hline 277 & 1.3 & 250 & 1.3 & & \\
\hline 299 & 1.6 & 300 & 1.2 & & \\
\hline 309 & 1.3 & 380 & 1.0 & & \\
\hline 327 & 1.2 & 421 & 1.2 & & \\
\hline 352 & 1.2 & 435 & 1.0 & & \\
\hline 366 & 1.1 & 460 & 1.2 & & \\
\hline 413 & 1.2 & 475 & 1.2 & & \\
\hline 463 & .85 & 491 & 1.3 & & \\
\hline 477 & 1.1 & 500 & 1.2 & & \\
\hline 487 & 1.2 & 521 & 1.2 & & \\
\hline 490 & 1.2 & 571 & 1.2 & & \\
\hline 521 & 1.4 & 593 & 1.2 & & \\
\hline 545 & 1.3 & $593 \mathrm{R}$ & 1.2 & & \\
\hline 576 & 1.2 & & & & \\
\hline 592 & 1.2 & & & & \\
\hline 630 & 1.2 & & & & \\
\hline 659 & 1.3 & & & & \\
\hline 673 & 1.2 & & & & \\
\hline 730 & 1.2 & & & & \\
\hline 752 & 1.6 & & & & \\
\hline 785 & 1.4 & & & & \\
\hline 816 & 1.4 & & & & \\
\hline 832 & 1.4 & & & & \\
\hline 870 & 1.4 & & & & \\
\hline 886 & 1.4 & & & & \\
\hline 923 & 1.3 & & & & \\
\hline $923 \mathrm{R}$ & 1.3 & & & & \\
\hline 961 & 1.4 & & & & \\
\hline 990 & 1.4 & & & & \\
\hline \multicolumn{6}{|c|}{ [Descriptive Statistics] } \\
\hline & & TAN CH 2 & TAN CH 1 & All cc & \\
\hline & Parameter & ICP/MS & ICP/MS & $\overline{\mid C P /}$ & \\
\hline & Minimum & 0.85 & 1.0 & 0.8 & \\
\hline & Maximum & 1.8 & 2.3 & 2.3 & \\
\hline & Mean & 1.4 & 1.4 & 1.3 & \\
\hline & Error & .03 & .08 & & \\
\hline & Median & 1.3 & 1.2 & 1.3 & \\
\hline & Error & .04 & .10 & & \\
\hline & Sample size & 35 & 18 & 56 & \\
\hline
\end{tabular}


L: Lanthanum (La)-Average crustal abundance is $35 \mathrm{ppm}$ (Emsley, 1989)

[La concentrations by XRF are qualitative estimates]

\begin{tabular}{|c|c|c|c|c|c|c|c|c|c|c|}
\hline \multicolumn{11}{|c|}{ [Concentration] } \\
\hline \multicolumn{3}{|c|}{ TAN CH 2} & \multicolumn{3}{|c|}{ TAN CH 1} & \multicolumn{3}{|c|}{ NPR Test } & \multicolumn{2}{|c|}{ NPA W01 } \\
\hline Depth & ICP/MS & XRF & Depth & ICP/MS & XRF & Depth & ICP/MS & XRF & Depth & XRF \\
\hline 138 & 50 & 50 & 61 & 68 & 69 & 36 & $\mathrm{ND}$ & 27 & 418 & 8.0 \\
\hline 154 & 47 & 50 & 91 & 45 & 34 & $36 \mathrm{R}$ & ND & 31 & 448 & 0 \\
\hline 172 & 39 & 40 & 111 & 37 & 49 & 56 & ND & 28 & $448 \mathrm{R}$ & 8.0 \\
\hline 201 & 37 & 30 & 130 & 39 & 31 & 98 & ND & 30 & 476 & 31 \\
\hline 215 & 39 & 38 & 150 & 39 & 39 & 121 & ND & 20 & 522 & 0 \\
\hline 238 & 36 & 47 & 195 & 41 & 27 & 127 & ND & 21 & & \\
\hline 256 & 42 & 55 & $195 \mathrm{R}$ & 40 & ND & 141 & ND & 32 & & \\
\hline 277 & 32 & 30 & 250 & 31 & 21 & 156 & ND & 26 & \multicolumn{2}{|c|}{ TRA 5} \\
\hline 299 & 39 & 51 & 300 & 26 & 33 & 170 & ND & 23 & Depth & XRF \\
\hline 309 & 31 & 40 & 380 & 18 & 26 & 232 & ND & 20 & 100 & 29 \\
\hline 327 & 28 & 24 & 421 & 22 & 23 & 273 & ND & 53 & 139 & 5.0 \\
\hline 352 & 27 & 24 & 435 & 19 & 0 & 291 & 26 & 34 & 162 & 0 \\
\hline 366 & 24 & 30 & 460 & 23 & 17 & 311 & ND & 20 & 170 & 32 \\
\hline 413 & 23 & 27 & 475 & 23 & 27 & 379 & ND & 30 & 192 & 55 \\
\hline 463 & 13 & 4.0 & 491 & 24 & 27 & 450 & 10 & 0 & 202 & 26 \\
\hline 477 & 18 & 9.0 & 500 & 22 & 20 & 461 & ND & 32 & & \\
\hline 487 & 25 & 25 & 521 & 17 & 0 & 480 & ND & 28 & & \\
\hline 490 & 24 & 21 & 571 & 18 & 12 & 489 & ND & 23 & & \\
\hline 521 & 27 & 23 & 593 & 18 & 19 & 527 & 12 & 7.0 & & \\
\hline 545 & 23 & 0 & $593 \mathrm{R}$ & 18 & ND & 538 & ND & 21 & & \\
\hline 576 & 18 & 15 & & & & 550 & ND & 27 & & \\
\hline 592 & 19 & 7.0 & & & & 608 & ND & 28 & & \\
\hline 630 & 18 & 10 & & & & & & & & \\
\hline 659 & 19 & 28 & & & & & & & & \\
\hline 673 & 20 & 29 & & & & & & & & \\
\hline 730 & 19 & 13 & & & & & & & & \\
\hline 752 & 29 & 33 & & & & & & & & \\
\hline 785 & 23 & 10 & & & & & & & & \\
\hline 816 & 24 & 19 & & & & & & & & \\
\hline 832 & 24 & 21 & & & & & & & & \\
\hline 870 & 23 & 29 & & & & & & & & \\
\hline 886 & 24 & 18 & & & & & & & & \\
\hline 923 & 23 & 26 & & & & & & & & \\
\hline $923 \mathrm{R}$ & 23 & ND & & & & & & & & \\
\hline 961 & 25 & 14 & & & & & & & & \\
\hline 990 & 24 & 26 & & & & & & & & \\
\hline
\end{tabular}

\begin{tabular}{|c|c|c|c|c|c|c|c|}
\hline \multirow[b]{2}{*}{ Parameter } & TAN & & \multicolumn{2}{|c|}{$\begin{array}{c}\text { [Descriptive Statistics] } \\
\text { TAN CH } 1\end{array}$} & \multirow{2}{*}{$\frac{\text { NPR Test }}{\text { XRF }}$} & \multicolumn{2}{|c|}{ All cores } \\
\hline & ICP/MS & $\overline{\mathrm{XRF}}$ & ICP/MS & XRF & & ICP/MS & XRF \\
\hline Minimum & 13 & 0 & 17 & 0 & 0 & 10 & 0 \\
\hline Maximum & 50 & 55 & 68 & 69 & 53 & 68 & 69 \\
\hline Mean & 27 & 26 & 29 & 26 & 25 & 27 & 25 \\
\hline Error & 1.5 & 2.4 & 3.1 & 3.8 & 2.2 & 1.4 & 1.6 \\
\hline Median & 24 & 26 & 23.5 & 26.5 & 27 & 24 & 26 \\
\hline Error & 1.9 & 3.0 & 3.9 & 4.8 & 2.8 & 1.8 & 1.9 \\
\hline Sample size & 35 & 35 & 18 & 18 & 21 & 56 & 84 \\
\hline
\end{tabular}


$\mathrm{M}$ : Lead ( $\mathrm{Pb}$ )_Average crustal abundance is $13 \mathrm{ppm}$ (Emsley, 1989)

\begin{tabular}{|c|c|c|c|c|c|c|c|c|c|c|}
\hline \multicolumn{11}{|c|}{ [Concentration] } \\
\hline \multicolumn{3}{|c|}{ TAN CH 2} & \multicolumn{3}{|c|}{ TAN CH 1} & \multicolumn{3}{|c|}{ NPR Test } & \multicolumn{2}{|c|}{ NPR WO1 } \\
\hline Depth & ICP/MS & $\overline{\text { XRF }}$ & Depth & ICP/MS & XRF & Depth & ICP/MS & $\overline{X R F}$ & Depth & $\overline{\mathrm{XRF}}$ \\
\hline 138 & 6.4 & 4 & 61 & 7.5 & 8 & 36 & ND & 4 & 418 & 4 \\
\hline 154 & 6.0 & 6 & 91 & 5.1 & 5 & $36 \mathrm{R}$ & ND & 6 & 448 & 5 \\
\hline 172 & 5.3 & 3 & 111 & 4.6 & 7 & 56 & ND & 7 & $448 \mathrm{R}$ & 4 \\
\hline 201 & 5.2 & 2 & 130 & 4.7 & 6 & 98 & ND & 6 & 476 & 5 \\
\hline 215 & 5.6 & 9 & 150 & 5.3 & 6 & 121 & ND & 2 & 522 & 4 \\
\hline 238 & 4.9 & 6 & 195 & 5.8 & 5 & 127 & ND & 5 & & \\
\hline 256 & 6.3 & 6 & $195 \mathrm{R}$ & 5.9 & ND & 141 & ND & 6 & & \\
\hline 277 & 4.8 & 7 & 250 & 4.5 & 7 & 156 & ND & 4 & \multicolumn{2}{|c|}{ TRA 5} \\
\hline 299 & 6.1 & 4 & 300 & 4.0 & 5 & 170 & ND & 6 & Depth & $\overline{\mathrm{XAF}}$ \\
\hline 309 & 4.2 & 6 & 380 & 2.9 & 3 & 232 & ND & 6 & 100 & 6 \\
\hline 327 & 4.4 & 4 & 421 & 3.4 & 4 & 273 & ND & 6 & 139 & 6 \\
\hline 352 & 4.3 & 5 & 435 & 3.2 & 2 & 291 & 3.9 & 5 & 162 & 4 \\
\hline 366 & 3.8 & 2 & 460 & 3.9 & 3 & 311 & ND & 3 & 170 & 3 \\
\hline 413 & 4.3 & 2 & 475 & 3.9 & 6 & 379 & ND & 3 & 192 & 7 \\
\hline 463 & 3.5 & 1 & 491 & 4.3 & 4 & 450 & 1.9 & 2 & 202 & 7 \\
\hline 477 & 3.0 & 3 & 500 & 3.9 & 5 & 461 & ND & 5 & & \\
\hline 487 & 4.3 & 6 & 521 & 3.5 & 6 & 480 & ND & 6 & & \\
\hline 490 & 4.2 & 3 & 571 & 3.5 & 8 & 489 & ND & 4 & & \\
\hline 521 & 5.1 & 7 & 593 & 3.3 & 5 & 527 & 2.1 & 2 & & \\
\hline 545 & 3.8 & 4 & $593 \mathrm{R}$ & 3.4 & ND & 538 & ND & 4 & & \\
\hline 576 & 3.6 & 8 & & & & 550 & ND & 3 & & \\
\hline 592 & 3.4 & 5 & & & & 608 & ND & 5 & & \\
\hline 630 & 3.4 & 1 & & & & & & & & \\
\hline 659 & 3.5 & 4 & & & & & & & & \\
\hline 673 & 5.5 & 6 & & & & & & & & \\
\hline 730 & 3.8 & 2 & & & & & & & & \\
\hline 752 & 4.4 & 6 & & & & & & & & \\
\hline 785 & 3.7 & 5 & & & & & & & & \\
\hline 816 & 3.7 & 4 & & & & & & & & \\
\hline 832 & 4.1 & 6 & & & & & & & & \\
\hline 870 & 5.0 & 3 & & & & & & & & \\
\hline 886 & 4.1 & 5 & & & & & & & & \\
\hline 923 & 4.2 & 3 & & & & & & & & \\
\hline $923 R$ & 4.2 & ND & & & & & & & & \\
\hline 961 & 4.3 & 5 & & & & & & & & \\
\hline 990 & 3.8 & 7 & & & & & & & & \\
\hline
\end{tabular}

\begin{tabular}{|c|c|c|c|c|c|c|c|}
\hline \multirow[b]{3}{*}{ Parameter } & \multicolumn{5}{|c|}{ [Descriptive Statistics] } & \multirow{2}{*}{\multicolumn{2}{|c|}{ All cores }} \\
\hline & \multicolumn{2}{|c|}{ TAN CH 2} & \multicolumn{2}{|c|}{ TAN CH 1} & \multirow{2}{*}{$\frac{\text { NPR Test }}{\text { XRF }}$} & & \\
\hline & ICP/MS & $\overline{X R F}$ & ICP/MS & $\overline{\text { XRF }}$ & & ICP/MS & XRF \\
\hline Minimum & 3.0 & 1.0 & 2.9 & 2.0 & 2.0 & 1.9 & 1.0 \\
\hline Maximum & 6.4 & 9.0 & 7.5 & 8.0 & 7.0 & 7.5 & 9.0 \\
\hline Mean & 4.5 & 4.6 & 4.3 & 5.3 & 4.5 & 4.3 & 4.8 \\
\hline Error & .15 & .33 & .26 & .39 & .34 & .14 & .19 \\
\hline Median & 4.3 & 5.0 & 3.95 & 5.0 & 5.0 & 4.2 & 5.0 \\
\hline Error & .19 & .42 & .33 & .49 & .42 & .18 & .24 \\
\hline Sample size & 35 & 35 & 18 & 18 & 21 & 56 & 84 \\
\hline
\end{tabular}


$\mathrm{N}$ : Lutetium (Lu)—Average crustal abundance is $0.8 \mathrm{ppm}$ (Emsley, 1989)

\begin{tabular}{|c|c|c|c|c|c|}
\hline \multicolumn{6}{|c|}{ [Concentration] } \\
\hline \multicolumn{2}{|c|}{ TAN CH 2} & \multicolumn{2}{|c|}{ TAN CH 1} & \multicolumn{2}{|c|}{ NPR Test } \\
\hline Depth & ICP/MS & Depth & ICP/MS & Depth & ICP/MS \\
\hline 138 & 0.71 & 61 & 0.87 & 291 & 0.47 \\
\hline 154 & .66 & 91 & .63 & 450 & .41 \\
\hline 172 & .63 & 111 & 60 & 527 & .39 \\
\hline 201 & .62 & 130 & .65 & & \\
\hline 215 & .64 & 150 & .67 & & \\
\hline 238 & .61 & 195 & .59 & & \\
\hline 256 & .55 & $195 \mathrm{R}$ & .57 & & \\
\hline 277 & .50 & 250 & .51 & & \\
\hline 299 & .62 & 300 & .50 & & \\
\hline 309 & .51 & 380 & .45 & & \\
\hline 327 & .48 & 421 & .47 & & \\
\hline 352 & .51 & 435 & .40 & & \\
\hline 366 & .45 & 460 & .48 & & \\
\hline 413 & .48 & 475 & .48 & & \\
\hline 463 & .35 & 491 & .52 & & \\
\hline 477 & .45 & 500 & .48 & & \\
\hline 487 & .47 & 521 & .47 & & \\
\hline 490 & .45 & 571 & .49 & & \\
\hline 521 & .54 & 593 & .48 & & \\
\hline 545 & .49 & $593 R$ & .48 & & \\
\hline 576 & .45 & & & & \\
\hline 592 & .49 & & & & \\
\hline 630 & .45 & & & & \\
\hline 659 & .50 & & & & \\
\hline 673 & .50 & & & & \\
\hline 730 & .47 & & & & \\
\hline 752 & .58 & & & & \\
\hline 785 & .52 & & & & \\
\hline 816 & .55 & & & & \\
\hline 832 & .54 & & & & \\
\hline 870 & .52 & & & & \\
\hline 886 & .55 & & & & \\
\hline 923 & .53 & & & & \\
\hline $923 \mathrm{R}$ & .52 & & & & \\
\hline 961 & .56 & & & & \\
\hline 990 & .53 & & & & \\
\hline \multicolumn{6}{|c|}{ [Descriptive Statistics] } \\
\hline & & TAN CH 2 & TAN CH 1 & All co & \\
\hline & Parameter & ICPMS & ICP/MS & ICP/ & \\
\hline & Minimum & 0.35 & 0.40 & $\overline{0.3}$ & \\
\hline & Maximum & .71 & .87 & .8 & \\
\hline & Mean & .53 & .54 & .5 & \\
\hline & Error & .01 & .03 & .0 & \\
\hline & Median & .52 & .495 & & \\
\hline & Error & .02 & .03 & .0 & \\
\hline & Sample size & 35 & 18 & 56 & \\
\hline
\end{tabular}


O: Neodymium (Nd)-Average crustal abundance is 40 ppm (Emsley, 1989)

\begin{tabular}{|c|c|c|c|c|c|}
\hline \multicolumn{6}{|c|}{ [Concentration] } \\
\hline \multicolumn{2}{|c|}{ TAN CH 2} & \multicolumn{2}{|c|}{ TAN CH 1} & \multicolumn{2}{|c|}{ NPR Test } \\
\hline Depth & ICP/MS & Depth & ICP/MS & Depth & ICP/MS \\
\hline 138 & 54 & 61 & 72 & 291 & 30 \\
\hline 154 & 51 & 91 & 48 & 450 & 13 \\
\hline 172 & 45 & 111 & 43 & 527 & 15 \\
\hline 201 & 43 & 130 & 46 & & \\
\hline 215 & 46 & 150 & 47 & & \\
\hline 238 & 43 & 195 & 45 & & \\
\hline 256 & 42 & $195 \mathrm{R}$ & 43 & & \\
\hline 277 & 35 & 250 & 35 & & \\
\hline 299 & 44 & 300 & 31 & & \\
\hline 309 & 35 & 380 & 22 & & \\
\hline 327 & 33 & 421 & 28 & & \\
\hline 352 & 32 & 435 & 23 & & \\
\hline 366 & 28 & 460 & 29 & & \\
\hline 413 & 29 & 475 & 29 & & \\
\hline 463 & 17 & 491 & 31 & & \\
\hline 477 & 23 & 500 & 29 & & \\
\hline 487 & 32 & 521 & 24 & & \\
\hline 490 & 31 & 571 & 25 & & \\
\hline 521 & 35 & 593 & 25 & & \\
\hline 545 & 30 & $593 R$ & 25 & & \\
\hline 576 & 24 & & & & \\
\hline 592 & 27 & & & & \\
\hline 630 & 25 & & & & \\
\hline 659 & 27 & & & & \\
\hline 673 & 27 & & & & \\
\hline 730 & 26 & & & & \\
\hline 752 & 39 & & & & \\
\hline 785 & 33 & & & & \\
\hline 816 & 34 & & & & \\
\hline 832 & 33 & & & & \\
\hline 870 & 32 & & & & \\
\hline 886 & 32 & & & & \\
\hline 923 & 30 & & & & \\
\hline $923 \mathrm{R}$ & 30 & & & & \\
\hline 961 & 32 & & & & \\
\hline 990 & 31 & & & & \\
\hline \multicolumn{6}{|c|}{ [Descriptive Statistics] } \\
\hline & & TAN CH 2 & TAN CH 1 & All c & \\
\hline & Parameter & ICP/MS & ICP/MS & ICP & \\
\hline & Minimum & 17 & 22 & & \\
\hline & Maximum & 54 & 72 & & \\
\hline & Mean & 34 & 35 & & \\
\hline & Error & 1.4 & 3.0 & & \\
\hline & Median & 32 & 30 & & \\
\hline & Error & 1.7 & 3.8 & & \\
\hline & Sample size & 35 & 18 & & \\
\hline
\end{tabular}


P: Nickel (Ni)—Average crustal abundance is 99 ppm (Emsley, 1989)

[Ni concentrations less than $30 \mathrm{ppm}$ are semiquantitative]

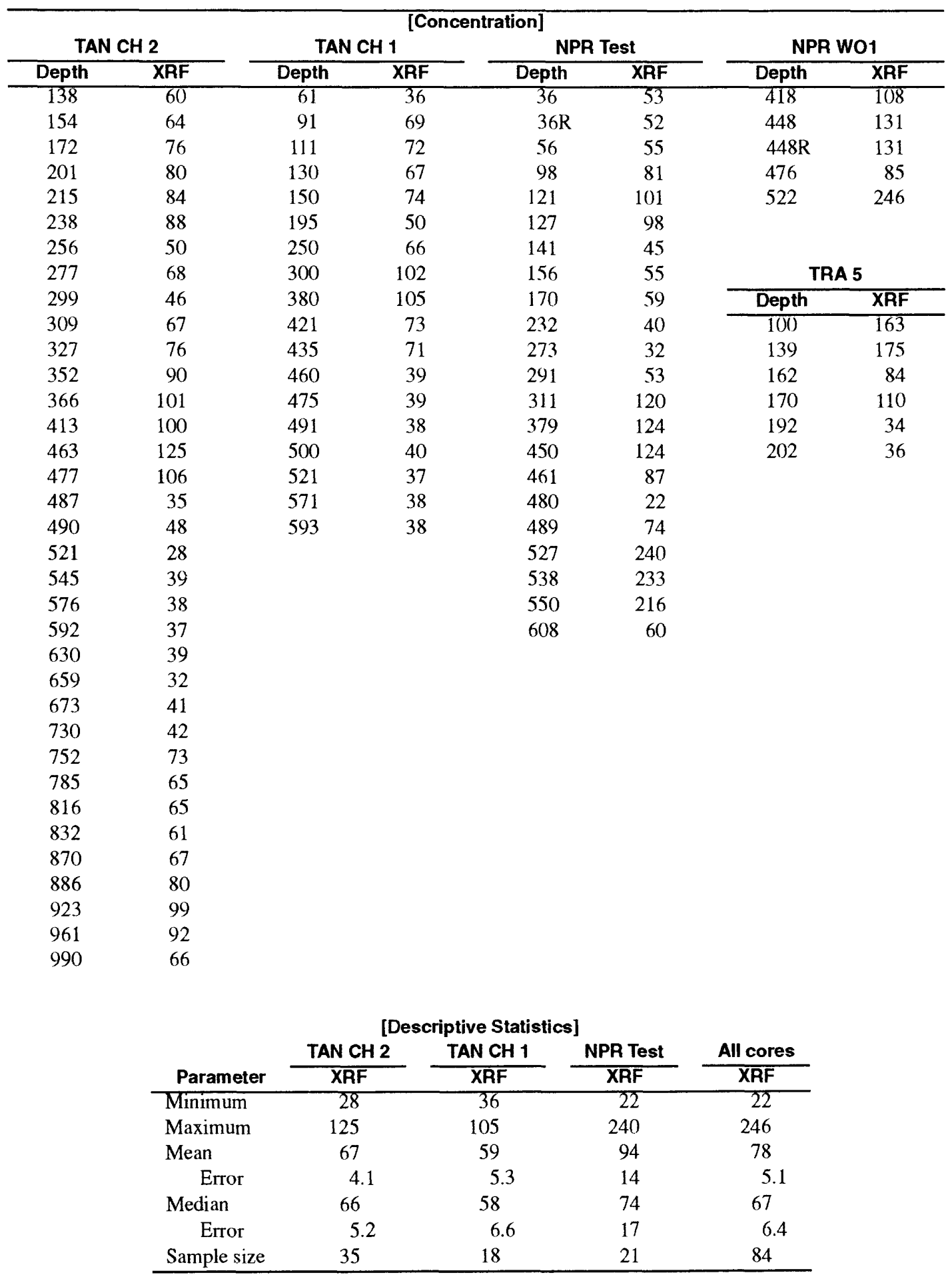


Q: Niobium ( $\mathrm{Nb}$ )-Average crustal abundance is $20 \mathrm{ppm}$ (Emsley, 1989)

\begin{tabular}{|c|c|c|c|c|c|c|c|c|c|c|}
\hline \multicolumn{11}{|c|}{ [Concentration] } \\
\hline \multicolumn{3}{|c|}{ TAN CH 2} & \multicolumn{3}{|c|}{ TAN CH 1} & \multicolumn{3}{|c|}{ NPR Test } & \multicolumn{2}{|c|}{ NPR W01 } \\
\hline Depth & ICP/MS & $\overline{\text { XRF }}$ & Depth & ICP/MS & $\overline{\mathrm{XRF}}$ & Depth & ICP/MS & XRF & Depth & $\overline{X R F}$ \\
\hline 138 & 48 & 46 & 61 & 63 & 58 & 36 & ND & 29 & 418 & 11 \\
\hline 154 & 42 & 42 & 91 & 43 & 38 & $36 \mathrm{R}$ & ND & 28 & 448 & 9.6 \\
\hline 172 & 39 & 35 & 111 & 37 & 37 & 56 & ND & 28 & $448 \mathrm{R}$ & 9.1 \\
\hline 201 & 38 & 36 & 130 & 40 & 37 & 98 & ND & 30 & 476 & 25 \\
\hline 215 & 40 & 37 & 150 & 41 & 37 & 121 & ND & 23 & 522 & 14 \\
\hline 238 & 37 & 34 & 195 & 42 & 38 & 127 & ND & 23 & & \\
\hline 256 & 41 & 37 & $195 \mathrm{R}$ & 39 & ND & 141 & ND & 31 & & \\
\hline 277 & 32 & 31 & 250 & 30 & 31 & 156 & ND & 34 & \multicolumn{2}{|c|}{ TRA 5} \\
\hline 299 & 39 & 36 & 300 & 26 & 25 & 170 & $\mathrm{ND}$ & 34 & Depth & $\overline{\text { XRF }}$ \\
\hline 309 & 31 & 30 & 380 & 18 & 16 & 232 & ND & 32 & 100 & 21 \\
\hline 327 & 27 & 27 & 421 & 22 & 22 & 273 & ND & 34 & 139 & 21 \\
\hline 352 & 26 & 27 & 435 & 20 & 18 & 291 & 27 & 26 & 162 & 18 \\
\hline 366 & 23 & 25 & 460 & 23 & 23 & 311 & ND & 19 & 170 & 16 \\
\hline 413 & 22 & 24 & 475 & 23 & 21 & 379 & ND & 17 & 192 & 36 \\
\hline 463 & 13 & 14 & 491 & 24 & 23 & 450 & 9.6 & 9.0 & 202 & 40 \\
\hline 477 & 18 & 18 & 500 & 23 & 23 & 461 & ND & 26 & & \\
\hline 487 & 26 & 27 & 521 & 14 & 17 & 480 & ND & 42 & & \\
\hline 490 & 24 & 24 & 571 & 18 & 18 & 489 & ND & 26 & & \\
\hline 521 & 28 & 26 & 593 & 18 & 20 & 527 & 12 & 13 & & \\
\hline 545 & 22 & 23 & 593R & 17 & $\mathrm{ND}$ & 538 & ND & 13 & & \\
\hline 576 & 18 & 18 & & & & 550 & ND & 10 & & \\
\hline 592 & 20 & 20 & & & & 608 & ND & 22 & & \\
\hline 630 & 18 & 19 & & & & & & & & \\
\hline 659 & 20 & 20 & & & & & & & & \\
\hline 673 & 19 & 20 & & & & & & & & \\
\hline 730 & 19 & 19 & & & & & & & & \\
\hline 752 & 26 & 27 & & & & & & & & \\
\hline 785 & 21 & 22 & & & & & & & & \\
\hline 816 & 22 & 22 & & & & & & & & \\
\hline 832 & 21 & 22 & & & & & & & & \\
\hline 870 & 20 & 20 & & & & & & & & \\
\hline 886 & 22 & 22 & & & & & & & & \\
\hline 923 & 22 & 22 & & & & & & & & \\
\hline $923 \mathrm{R}$ & 21 & ND & & & & & & & & \\
\hline 961 & 24 & 22 & & & & & & & & \\
\hline 990 & 27 & 28 & & & & & & & & \\
\hline
\end{tabular}

\begin{tabular}{|c|c|c|c|c|c|c|c|}
\hline \multirow[b]{3}{*}{ Parameter } & \multicolumn{5}{|c|}{ [Descriptive Statistics] } & \multirow{2}{*}{\multicolumn{2}{|c|}{ All cores }} \\
\hline & \multicolumn{2}{|c|}{ TAN CH 2} & \multicolumn{2}{|c|}{ TAN CH 1} & \multirow{2}{*}{$\frac{\text { NPR Test }}{\text { XRF }}$} & & \\
\hline & ICP/MS & XRF & ICP/MS & $\overline{\text { XRF }}$ & & ICP/MS & XRF \\
\hline Minimum & 13 & 14 & 14 & 16 & 9.0 & 10 & 9.0 \\
\hline Maximum & 48 & 46 & 63 & 58 & 42 & 63 & 58 \\
\hline Mean & 27 & 26 & 29 & 28 & 25 & 27 & 26 \\
\hline Error & 1.5 & 1.3 & 3.0 & 2.6 & 1.9 & 1.4 & .99 \\
\hline Median & 24 & 24 & 23.5 & 23 & 26 & 23.5 & 23.5 \\
\hline Error & 1.8 & 1.6 & 3.7 & 3.3 & 2.4 & 1.7 & 1.2 \\
\hline Sample size & 35 & 35 & 18 & 18 & 21 & 56 & 84 \\
\hline
\end{tabular}


R: Praseodymium (Pr)-Average crustal abundance is $9.1 \mathrm{ppm}$ (Emsley, 1989)

\begin{tabular}{|c|c|c|c|c|c|}
\hline \multicolumn{6}{|c|}{ [Concentration] } \\
\hline Depth & ICP/MS & Depth & ICP/MS & Depth & ICP/MS \\
\hline 138 & 13 & 61 & 18 & 291 & 7.3 \\
\hline 154 & 12 & 91 & 12 & 450 & 2.9 \\
\hline 172 & 11 & 111 & 10 & 527 & 3.6 \\
\hline 201 & 10 & 130 & 11 & & \\
\hline 215 & 11 & 150 & 11 & & \\
\hline 238 & 10 & 195 & 11 & & \\
\hline 256 & 10 & $195 \mathrm{R}$ & 10 & & \\
\hline 277 & 8.6 & 250 & 8.2 & & \\
\hline 299 & 10 & 300 & 7.3 & & \\
\hline 309 & 8.2 & 380 & 5.1 & & \\
\hline 327 & 7.8 & 421 & 6.3 & & \\
\hline 352 & 7.5 & 435 & 5.4 & & \\
\hline 366 & 6.7 & 460 & 6.7 & & \\
\hline 413 & 6.6 & 475 & 6.7 & & \\
\hline 463 & 4.0 & 491 & 7.0 & & \\
\hline 477 & 5.3 & 500 & 6.5 & & \\
\hline 487 & 7.3 & 521 & 5.2 & & \\
\hline 490 & 7.0 & 571 & 5.6 & & \\
\hline 521 & 7.9 & 593 & 5.6 & & \\
\hline 545 & 6.9 & $593 \mathrm{R}$ & 5.5 & & \\
\hline 576 & 5.4 & & & & \\
\hline 592 & 5.9 & & & & \\
\hline 630 & 5.4 & & & & \\
\hline 659 & 6.0 & & & & \\
\hline 673 & 6.1 & & & & \\
\hline 730 & 5.7 & & & & \\
\hline 752 & 8.7 & & & & \\
\hline 785 & 7.2 & & & & \\
\hline 816 & 7.5 & & & & \\
\hline 832 & 7.2 & & & & \\
\hline 870 & 7.1 & & & & \\
\hline 886 & 7.1 & & & & \\
\hline 923 & 6.8 & & & & \\
\hline $923 R$ & 6.8 & & & & \\
\hline 961 & 7.3 & & & & \\
\hline 990 & 6.9 & & & & \\
\hline \multicolumn{6}{|c|}{ [Descriptive Statistics] } \\
\hline & & TAN CH 2 & TAN CH 1 & All $\mathrm{c}$ & \\
\hline & Parameter & ICP/MS & ICP/MS & TCP] & \\
\hline & Minimum & 4.0 & 5.1 & 2. & \\
\hline & Maximum & 13 & 17 & 17 & \\
\hline & Mean & 7.7 & 8.2 & 7. & \\
\hline & Error & .34 & .75 & & \\
\hline & Median & 7.2 & 6.85 & 7. & \\
\hline & Error & .43 & .94 & & \\
\hline & Sample size & 35 & 18 & 56 & \\
\hline
\end{tabular}


S: Rubidium (Rb) - Average crustal abundance is 78 ppm (Emsley, 1989)

[ $\mathrm{Rb}$ concentrations less than $3 \mathrm{ppm}$ are semiquantitative]

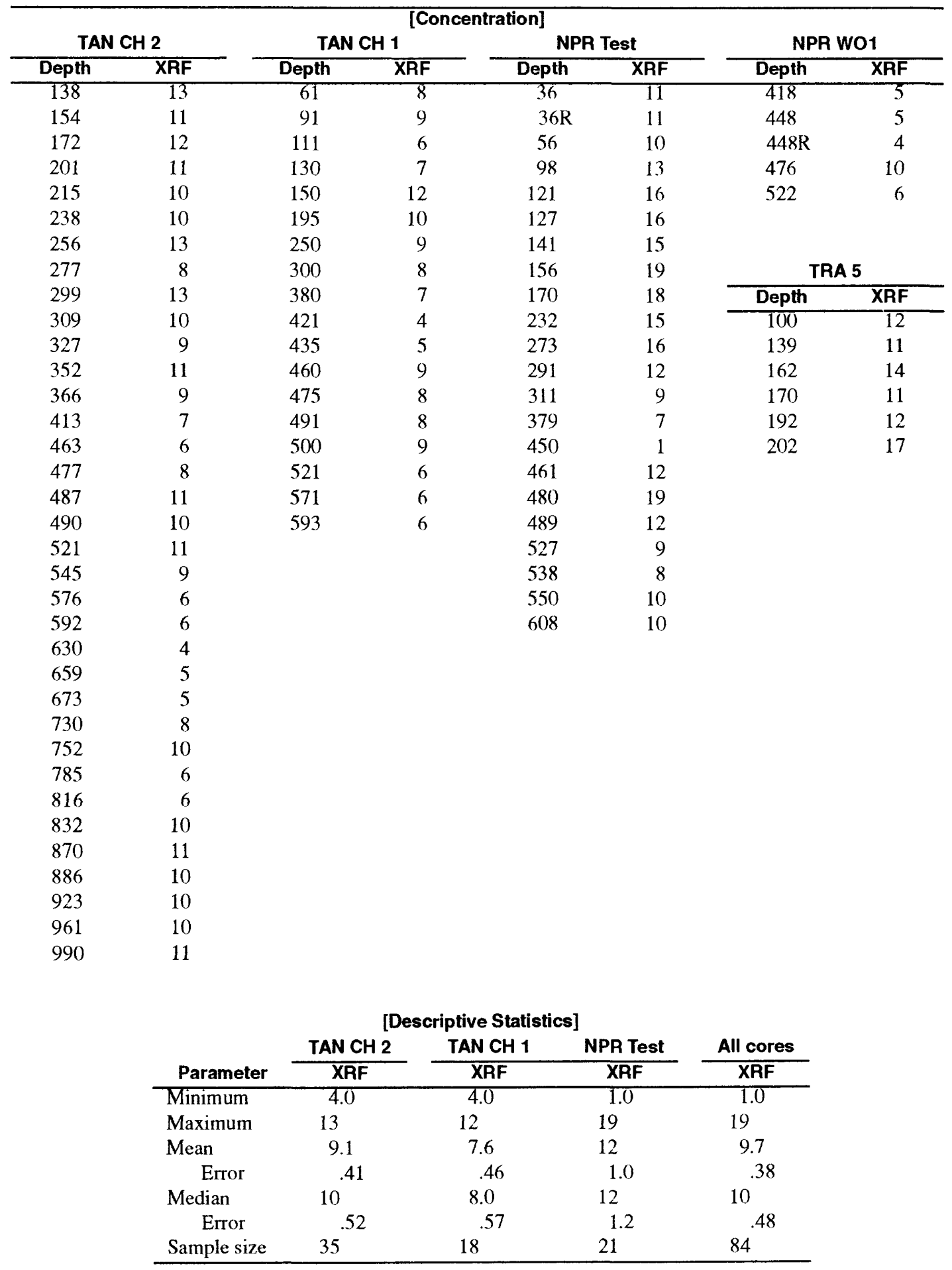


T: Samarium (Sm)—Average crustal abundance is $7.0 \mathrm{ppm}$ (Emsley, 1989)

\begin{tabular}{|c|c|c|c|c|c|}
\hline \multicolumn{6}{|c|}{ [Concentration] } \\
\hline Depth & ICP/MS & Depth & TCP/MS & Depth & $\overline{\mathrm{ICP} /}$ \\
\hline 138 & 11 & 61 & 15 & 291 & 6.7 \\
\hline 154 & 11 & 91 & 10 & 450 & 3.2 \\
\hline 172 & 9.8 & 111 & 9.5 & 527 & 3.6 \\
\hline 201 & 9.2 & 130 & 10 & & \\
\hline 215 & 9.8 & 150 & 10 & & \\
\hline 238 & 9.4 & 195 & 9.4 & & \\
\hline 256 & 8.6 & $195 \mathrm{R}$ & 9.0 & & \\
\hline 277 & 7.6 & 250 & 7.7 & & \\
\hline 299 & 9.6 & 300 & 7.0 & & \\
\hline 309 & 7.5 & 380 & 5.2 & & \\
\hline 327 & 7.3 & 421 & 6.4 & & \\
\hline 352 & 7.1 & 435 & 5.3 & & \\
\hline 366 & 6.4 & 460 & 6.8 & & \\
\hline 413 & 6.5 & 475 & 6.8 & & \\
\hline 463 & 4.2 & 491 & 7.2 & & \\
\hline 477 & 5.4 & 500 & 6.6 & & \\
\hline 487 & 7.4 & 521 & 5.8 & & \\
\hline 490 & 7.0 & 571 & 6.2 & & \\
\hline 521 & 8.3 & 593 & 6.1 & & \\
\hline 545 & 7.2 & $593 R$ & 5.9 & & \\
\hline 576 & 6.0 & & & & \\
\hline 592 & 6.4 & & & & \\
\hline 630 & 6.0 & & & & \\
\hline 659 & 6.6 & & & & \\
\hline 673 & 6.5 & & & & \\
\hline 730 & 6.2 & & & & \\
\hline 752 & 8.9 & & & & \\
\hline 785 & 7.7 & & & & \\
\hline 816 & 8.0 & & & & \\
\hline 832 & 7.8 & & & & \\
\hline 870 & 7.5 & & & & \\
\hline 886 & 7.4 & & & & \\
\hline 923 & 6.9 & & & & \\
\hline 923R & 7.0 & & & & \\
\hline 961 & 7.6 & & & & \\
\hline 990 & 7.2 & & & & \\
\hline \multicolumn{6}{|c|}{ [Descriptive Statistics] } \\
\hline & & TAN CH 2 & TAN CH 1 & All c & \\
\hline & Parameter & ICP/MS & ICP/MS & ICF & \\
\hline & Minimum & 4.2 & 5.2 & & \\
\hline & Maximum & 11 & 15 & 15 & \\
\hline & Mean & 7.6 & 7.8 & & \\
\hline & Error & .26 & .57 & & \\
\hline & Median & 7.4 & 6.9 & & \\
\hline & Error & .32 & .72 & & \\
\hline & Sample size & 35 & 18 & 56 & \\
\hline
\end{tabular}


$\mathrm{U}$ : Scandium (Sc) - Average crustal abundance is $25 \mathrm{ppm}$ (Emsley, 1989)

[Sc concentrations less than $30 \mathrm{ppm}$ are semiquantitative]

\begin{tabular}{|c|c|c|c|c|c|c|c|}
\hline \multicolumn{8}{|c|}{ [Concentration] } \\
\hline \multicolumn{2}{|c|}{ TAN CH 2} & \multicolumn{2}{|c|}{ TAN CH 1} & \multicolumn{2}{|c|}{ NPR Test } & \multicolumn{2}{|c|}{ NPR WO1 } \\
\hline Depth & XRF & Depth & & Depth & $\overline{\mathbf{X R F}}$ & Depth & XRF \\
\hline 138 & 31 & 61 & & 36 & 35 & 418 & 40 \\
\hline 154 & 31 & 91 & & $36 \mathrm{R}$ & 34 & 448 & 34 \\
\hline 172 & 34 & 111 & & 56 & 26 & $448 \mathrm{R}$ & 36 \\
\hline 201 & 31 & 130 & & 98 & 30 & 476 & 31 \\
\hline 215 & 26 & 150 & & 121 & 30 & 522 & 33 \\
\hline 238 & 31 & 195 & & 127 & 32 & & \\
\hline 256 & 24 & 250 & & 141 & 34 & & \\
\hline 277 & 27 & 300 & & 156 & 31 & \multicolumn{2}{|c|}{ TRA 5} \\
\hline 299 & 40 & 380 & & 170 & 30 & Depth & XRF \\
\hline 309 & 28 & 421 & & 232 & 33 & 100 & 36 \\
\hline 327 & 30 & 435 & & 273 & 33 & 139 & 34 \\
\hline 352 & 28 & 460 & & 291 & 29 & 162 & 34 \\
\hline 366 & 26 & 475 & & 311 & 37 & 170 & 28 \\
\hline 413 & 31 & 491 & & 379 & 38 & 192 & 37 \\
\hline 463 & 37 & 500 & & 450 & 37 & 202 & 43 \\
\hline 477 & 34 & 521 & & 461 & 32 & & \\
\hline 487 & 28 & 571 & & 480 & 43 & & \\
\hline 490 & 25 & 593 & & 489 & 32 & & \\
\hline 521 & 35 & & & 527 & 41 & & \\
\hline 545 & 31 & & & 538 & 38 & & \\
\hline 576 & 30 & & & 550 & 38 & & \\
\hline 592 & 33 & & & 608 & 35 & & \\
\hline 630 & 30 & & & & & & \\
\hline 659 & 33 & & & & & & \\
\hline 673 & 35 & & & & & & \\
\hline 730 & 35 & & & & & & \\
\hline 752 & 33 & & & & & & \\
\hline 785 & 27 & & & & & & \\
\hline 816 & 32 & & & & & & \\
\hline 832 & 31 & & & & & & \\
\hline 870 & 32 & & & & & & \\
\hline 886 & 34 & & & & & & \\
\hline 923 & 33 & & & & & & \\
\hline 961 & 35 & & & & & & \\
\hline 990 & 29 & & & & & & \\
\hline \multicolumn{8}{|c|}{ [Descriptive Statistics] } \\
\hline & & TAN & & TAN CH 1 & NPR Test & All cores & \\
\hline & Parameter & & & XRF & XRF & XRF & \\
\hline & Minimum & 24 & & 26 & 26 & 24 & \\
\hline & Maximum & 40 & & 43 & 43 & 43 & \\
\hline & Mean & 31 & & 33 & 34 & 33 & \\
\hline & Error & & & 1.4 & .92 & .49 & \\
\hline & Median & 31 & & 31.5 & 33 & 32 & \\
\hline & Error & & & 1.7 & 1.2 & .62 & \\
\hline & Sample size & 35 & & 18 & 21 & 84 & \\
\hline
\end{tabular}


$\mathrm{V}$ : Strontium (Sr)-Average crustal abundance is $384 \mathrm{ppm}$ (Emsley, 1989)

\begin{tabular}{|c|c|c|c|c|c|c|c|}
\hline \multicolumn{8}{|c|}{ [Concentration] } \\
\hline \multicolumn{2}{|c|}{ TAN CH 2} & \multicolumn{2}{|c|}{ TAN CH 1} & \multicolumn{2}{|c|}{ NPR Test } & \multicolumn{2}{|c|}{ NPR W01 } \\
\hline Depth & XRF & Depth & XRF & Depth & XRF & Depth & XRF \\
\hline 138 & 300 & 61 & 306 & 36 & 313 & 418 & 187 \\
\hline 154 & 301 & 91 & 313 & $36 \mathrm{R}$ & 308 & 448 & 188 \\
\hline 172 & 315 & 111 & 329 & 56 & 311 & $448 \mathrm{R}$ & 185 \\
\hline 201 & 312 & 130 & 330 & 98 & 287 & 476 & 340 \\
\hline 215 & 317 & 150 & 319 & 121 & 258 & 522 & 203 \\
\hline 238 & 319 & 195 & 320 & 127 & 260 & & \\
\hline 256 & 327 & 250 & 332 & 141 & 304 & & \\
\hline 277 & 330 & 300 & 298 & 156 & 299 & \multicolumn{2}{|c|}{ TRA 5} \\
\hline 299 & 315 & 380 & 241 & 170 & 297 & Depth & $\overline{X R F}$ \\
\hline 309 & 325 & 421 & 270 & 232 & 295 & 100 & 269 \\
\hline 327 & 315 & 435 & 268 & 273 & 292 & 139 & 264 \\
\hline 352 & 292 & 460 & 362 & 291 & 311 & 162 & 251 \\
\hline 366 & 292 & 475 & 366 & 311 & 226 & 170 & 249 \\
\hline 413 & 289 & 491 & 349 & 379 & 205 & 192 & 279 \\
\hline 463 & 226 & 500 & 348 & 450 & 190 & 202 & 273 \\
\hline 477 & 241 & 521 & 315 & 461 & 342 & & \\
\hline 487 & 381 & 571 & 315 & 480 & 300 & & \\
\hline 490 & 392 & 593 & 316 & 489 & 341 & & \\
\hline 521 & 363 & & & 527 & 197 & & \\
\hline 545 & 357 & & & 538 & 199 & & \\
\hline 576 & 324 & & & 550 & 196 & & \\
\hline 592 & 308 & & & 608 & 249 & & \\
\hline 630 & 310 & & & & & & \\
\hline 659 & 308 & & & & & & \\
\hline 673 & 312 & & & & & & \\
\hline 730 & 311 & & & & & & \\
\hline 752 & 304 & & & & & & \\
\hline 785 & 329 & & & & & & \\
\hline 816 & 322 & & & & & & \\
\hline 832 & 319 & & & & & & \\
\hline 870 & 319 & & & & & & \\
\hline 886 & 293 & & & & & & \\
\hline 923 & 294 & & & & & & \\
\hline 961 & 292 & & & & & & \\
\hline 990 & 362 & & & & & & \\
\hline
\end{tabular}

[Descriptive Statistics]

\begin{tabular}{|c|c|c|c|c|}
\hline Parameter & $\frac{\text { TAN CH } 2}{X A F}$ & $\frac{\text { TAN CH } 1}{\text { XRF }}$ & $\frac{\text { NPR Test }}{\text { XRF }}$ & $\frac{\text { All cores }}{\text { XRF }}$ \\
\hline Minimum & 226 & 241 & 190 & 187 \\
\hline Maximum & 392 & 366 & 342 & 392 \\
\hline Mean & 315 & 316 & 270 & 296 \\
\hline Error & 5.4 & 7.6 & 11 & 5.0 \\
\hline Median & 315 & 317.5 & 292 & 308 \\
\hline Error & 6.8 & 9.6 & 14 & 6.3 \\
\hline Sample size & 35 & 18 & 21 & 84 \\
\hline
\end{tabular}


W: Tantalum (Ta) - Average crustal abundance is $1.7 \mathrm{ppm}$ (Emsley, 1989)

\begin{tabular}{|c|c|c|c|c|c|}
\hline \multicolumn{6}{|c|}{ [Concentration] } \\
\hline Depth & ICP/MS & Depth & ICP/MS & Depth & ICP/MS \\
\hline 138 & 2.8 & 61 & 3.3 & 291 & 2.1 \\
\hline 154 & 2.0 & 91 & 2.0 & 450 & .87 \\
\hline 172 & 2.4 & 111 & 2.4 & 527 & 1.3 \\
\hline 201 & 2.0 & 130 & 2.2 & & \\
\hline 215 & 2.5 & 150 & 2.5 & & \\
\hline 238 & 2.0 & 195 & 2.3 & & \\
\hline 256 & 2.5 & 195R & 2.3 & & \\
\hline 277 & 1.7 & 250 & 2.0 & & \\
\hline 299 & 2.2 & 300 & 1.6 & & \\
\hline 309 & 1.8 & 380 & 1.3 & & \\
\hline 327 & 1.7 & 421 & 1.4 & & \\
\hline 352 & 1.6 & 435 & 1.6 & & \\
\hline 366 & 1.8 & 460 & 1.4 & & \\
\hline 413 & 1.5 & 475 & 1.8 & & \\
\hline 463 & 1.6 & 491 & 1.6 & & \\
\hline 477 & 1.4 & 500 & 1.7 & & \\
\hline 487 & 1.7 & 521 & .94 & & \\
\hline 490 & 1.7 & 571 & 1.4 & & \\
\hline 521 & 1.7 & 593 & 1.2 & & \\
\hline 545 & 1.5 & $593 \mathrm{R}$ & 1.2 & & \\
\hline 576 & 1.7 & & & & \\
\hline 592 & 1.7 & & & & \\
\hline 630 & 1.4 & & & & \\
\hline 659 & 1.7 & & & & \\
\hline 673 & 1.3 & & & & \\
\hline 730 & 1.6 & & & & \\
\hline 752 & 1.7 & & & & \\
\hline 785 & 1.6 & & & & \\
\hline 816 & 1.4 & & & & \\
\hline 832 & 1.6 & & & & \\
\hline 870 & 1.4 & & & & \\
\hline 886 & 1.6 & & & & \\
\hline 923 & 1.3 & & & & \\
\hline $923 \mathrm{R}$ & 1.2 & & & & \\
\hline 961 & 1.6 & & & & \\
\hline 990 & 1.7 & & & & \\
\hline \multicolumn{6}{|c|}{ [Descriptive Statistics] } \\
\hline & & TAN CH 2 & TAN CH 1 & All $\mathrm{c}$ & \\
\hline & Parameter & ICP/MS & ICP/MS & |CPI & \\
\hline & Minimum & 1.3 & 0.94 & 0.8 & \\
\hline & Maximum & 2.8 & 3.3 & 3. & \\
\hline & Mean & 1.8 & 1.8 & 1.8 & \\
\hline & Error & .06 & .13 & & \\
\hline & Median & 1.7 & 1.65 & 1. & \\
\hline & Error & .07 & .17 & & \\
\hline & Sample size & 35 & 18 & 56 & \\
\hline
\end{tabular}


$\mathrm{X}$ : Terbium (Tb)-Average crustal abundance is $1.2 \mathrm{ppm}$ (Emsley, 1989)

\begin{tabular}{|c|c|c|c|c|c|}
\hline \multicolumn{6}{|c|}{ [Concentration] } \\
\hline Depth & ICP/MS & Depth & ICP/MS & Depth & ICP/MS \\
\hline 138 & 1.6 & 61 & 2.0 & 291 & 0.99 \\
\hline 154 & 1.5 & 91 & 1.5 & 450 & .62 \\
\hline 172 & 1.4 & 111 & 1.4 & 527 & .62 \\
\hline 201 & 1.4 & 130 & 1.4 & & \\
\hline 215 & 1.4 & 150 & 1.5 & & \\
\hline 238 & 1.4 & 195 & 1.4 & & \\
\hline 256 & 1.2 & $195 \mathrm{R}$ & 1.3 & & \\
\hline 277 & 1.1 & 250 & 1.1 & & \\
\hline 299 & 1.4 & 300 & 1.1 & & \\
\hline 309 & 1.1 & 380 & .85 & & \\
\hline 327 & 1.1 & 421 & .99 & & \\
\hline 352 & 1.1 & 435 & .84 & & \\
\hline 366 & .97 & 460 & .99 & & \\
\hline 413 & 1.0 & 475 & 1.0 & & \\
\hline 463 & .69 & 491 & 1.1 & & \\
\hline 477 & .88 & 500 & 1.0 & & \\
\hline 487 & 1.1 & 521 & .96 & & \\
\hline 490 & 1.0 & 571 & 1.0 & & \\
\hline 521 & 1.2 & 593 & 1.0 & & \\
\hline 545 & 1.1 & $593 \mathrm{R}$ & .98 & & \\
\hline 576 & .94 & & & & \\
\hline 592 & 1.0 & & & & \\
\hline 630 & .97 & & & & \\
\hline 659 & 1.0 & & & & \\
\hline 673 & 1.1 & & & & \\
\hline 730 & .99 & & & & \\
\hline 752 & 1.3 & & & & \\
\hline 785 & 1.2 & & & & \\
\hline 816 & 1.2 & & & & \\
\hline 832 & 1.2 & & & & \\
\hline 870 & 1.1 & & & & \\
\hline 886 & 1.2 & & & & \\
\hline 923 & 1.1 & & & & \\
\hline 923R & 1.1 & & & & \\
\hline 961 & 1.2 & & & & \\
\hline 990 & 1.1 & & & & \\
\hline \multicolumn{6}{|c|}{ [Descriptive Statistics] } \\
\hline & & TAN CH 2 & TAN CH 1 & All core & \\
\hline & Parameter & ICP/MS & ICP/MS & ICP/MS & \\
\hline & Minimum & 0.69 & 0.84 & 0.62 & \\
\hline & Maximum & 1.6 & 2.0 & 2.0 & \\
\hline & Mean & 1.1 & 1.2 & 1.1 & \\
\hline & Error & .03 & .07 & .03 & \\
\hline & Median & 1.1 & 1.05 & 1.1 & \\
\hline & Error & .04 & .09 & .04 & \\
\hline & Sample size & 35 & 18 & 56 & \\
\hline
\end{tabular}


Y: Thorium (Th)—Average crustal abundance is $8.1 \mathrm{ppm}$ (Emsley, 1989)

[Th XRF concentrations less than $3 \mathrm{ppm}$ are semiquantitative]

\begin{tabular}{|c|c|c|c|c|c|c|c|c|c|c|}
\hline \multicolumn{11}{|c|}{ [Concentration] } \\
\hline \multicolumn{3}{|c|}{ TAN CH 2} & \multicolumn{3}{|c|}{ TAN CH 1} & \multicolumn{3}{|c|}{ NPR Test } & \multicolumn{2}{|c|}{ NPR W01 } \\
\hline Depth & ICP/MS & $\overline{X R F}$ & Depth & ICP/MS & XRF & Depth & ICP/MS & $\overline{X R F}$ & Depth & XRF \\
\hline 138 & 2.1 & 4 & 61 & 2.1 & 2 & 36 & ND & 2 & 418 & 0 \\
\hline 154 & 1.9 & 3 & 91 & 1.8 & 4 & $36 \mathrm{R}$ & ND & 2 & 448 & 2 \\
\hline 172 & 1.6 & 1 & 111 & 1.1 & 0 & 56 & ND & 0 & $448 \mathrm{R}$ & 3 \\
\hline 201 & 1.5 & 7 & 130 & 1.2 & 0 & 98 & ND & 3 & 476 & 0 \\
\hline 215 & 1.6 & 4 & 150 & 1.6 & 3 & 121 & ND & 3 & 522 & 0 \\
\hline 238 & 1.4 & 1 & 195 & 1.7 & 1 & 127 & ND & 1 & & \\
\hline 256 & 1.9 & 2 & $195 \mathrm{R}$ & 1.6 & ND & 141 & ND & 4 & & \\
\hline 277 & 1.3 & 0 & 250 & 1.2 & 3 & 156 & ND & 2 & TR & \\
\hline 299 & 1.6 & 1 & 300 & 1.1 & 3 & 170 & ND & 3 & Depth & $\overline{\text { XRF }}$ \\
\hline 309 & 1.2 & 1 & 380 & .85 & 0 & 232 & ND & 3 & 100 & 1 \\
\hline 327 & 1.2 & 1 & 421 & .87 & 2 & 273 & ND & 2 & 139 & 2 \\
\hline 352 & 1.1 & 2 & 435 & .78 & 0 & 291 & 1.2 & 0 & 162 & 1 \\
\hline 366 & .98 & 0 & 460 & 1.1 & 0 & 311 & ND & 0 & 170 & 0 \\
\hline 413 & .99 & 1 & 475 & 1.0 & 1 & 379 & ND & 0 & 192 & 3 \\
\hline 463 & .68 & 0 & 491 & 1.2 & 0 & 450 & .60 & 2 & 202 & 5 \\
\hline 477 & .85 & 2 & 500 & 1.0 & 1 & 461 & ND & 4 & & \\
\hline 487 & 1.1 & 0 & 521 & .82 & 0 & 480 & ND & 0 & & \\
\hline 490 & 1.0 & 3 & 571 & .75 & 0 & 489 & ND & 3 & & \\
\hline 521 & 1.2 & 1 & 593 & .75 & 0 & 527 & .90 & 2 & & \\
\hline 545 & 1.0 & 1 & 593R & .69 & ND & 538 & ND & 0 & & \\
\hline 576 & .80 & 1 & & & & 550 & ND & 1 & & \\
\hline 592 & .70 & 2 & & & & 608 & ND & 0 & & \\
\hline 630 & .73 & 0 & & & & & & & & \\
\hline 659 & .77 & 1 & & & & & & & & \\
\hline 673 & .86 & 3 & & & & & & & & \\
\hline 730 & .86 & 3 & & & & & & & & \\
\hline 752 & 1.0 & 2 & & & & & & & & \\
\hline 785 & .64 & 1 & & & & & & & & \\
\hline 816 & .75 & 2 & & & & & & & & \\
\hline 832 & .95 & 1 & & & & & & & & \\
\hline 870 & 1.0 & 1 & & & & & & & & \\
\hline 886 & 1.3 & 0 & & & & & & & & \\
\hline 923 & 1.4 & 1 & & & & & & & & \\
\hline $923 R$ & 1.3 & ND & & & & & & & & \\
\hline 961 & 1.2 & 0 & & & & & & & & \\
\hline 990 & 1.4 & 1 & & & & & & & & \\
\hline
\end{tabular}

\begin{tabular}{|c|c|c|c|c|c|c|c|}
\hline \multirow[b]{2}{*}{ Parameter } & \multicolumn{2}{|c|}{ TAN CH 2} & \multicolumn{2}{|c|}{$\begin{array}{c}\text { [Descriptive Statistics] } \\
\text { TAN CH } 1\end{array}$} & \multirow{2}{*}{$\frac{\text { NPA Test }}{\text { XRF }}$} & \multicolumn{2}{|c|}{ All cores } \\
\hline & ICP/MS & XRF & ICP/MS & XRF & & ICP/MS & XRF \\
\hline Minimum & 0.64 & 0 & 0.75 & 0 & 0 & 0.60 & 0 \\
\hline Maximum & 2.1 & 7.0 & 2.1 & 4.0 & 4.0 & 2.1 & 7.0 \\
\hline Mean & 1.2 & 1.5 & 1.2 & 1.1 & 1.7 & 1.2 & 1.5 \\
\hline Error & .06 & .25 & .09 & .32 & .31 & .05 & .16 \\
\hline Median & 1.1 & 1.0 & 1.1 & .50 & 2.0 & 1.1 & 1.0 \\
\hline Error & .08 & .31 & .12 & .40 & .39 & .06 & .20 \\
\hline Sample size & 35 & 35 & 18 & 18 & 21 & 56 & 84 \\
\hline
\end{tabular}


Z: Thulium (Tm)-Average crustal abundance is $0.5 \mathrm{ppm}$ (Emsley, 1989)

\begin{tabular}{|c|c|c|c|c|c|}
\hline \multicolumn{6}{|c|}{ [Concentration] } \\
\hline Depth & ICP/MS & Depth & ICP/MS & Depth & ICP/MS \\
\hline 138 & 0.75 & 61 & 0.89 & 291 & 0.48 \\
\hline 154 & .72 & 91 & .67 & 450 & .37 \\
\hline 172 & .67 & 111 & .63 & 527 & .38 \\
\hline 201 & .66 & 130 & .66 & & \\
\hline 215 & .69 & 150 & .70 & & \\
\hline 238 & .65 & 195 & .61 & & \\
\hline 256 & .57 & $195 \mathrm{R}$ & .60 & & \\
\hline 277 & .52 & 250 & .53 & & \\
\hline 299 & .67 & 300 & .51 & & \\
\hline 309 & .53 & 380 & .44 & & \\
\hline 327 & .53 & 421 & .47 & & \\
\hline 352 & .54 & 435 & .40 & & \\
\hline 366 & .47 & 460 & .48 & & \\
\hline 413 & .50 & 475 & .49 & & \\
\hline 463 & .36 & 491 & .53 & & \\
\hline 477 & .46 & 500 & .49 & & \\
\hline 487 & .50 & 521 & .48 & & \\
\hline 490 & .49 & 571 & .50 & & \\
\hline 521 & .59 & 593 & .50 & & \\
\hline 545 & .52 & $593 R$ & .48 & & \\
\hline 576 & .48 & & & & \\
\hline 592 & .52 & & & & \\
\hline 630 & .50 & & & & \\
\hline 659 & .52 & & & & \\
\hline 673 & .53 & & & & \\
\hline 730 & .50 & & & & \\
\hline 752 & .62 & & & & \\
\hline 785 & .56 & & & & \\
\hline 816 & .59 & & & & \\
\hline 832 & .57 & & & & \\
\hline 870 & .56 & & & & \\
\hline 886 & .57 & & & & \\
\hline 923 & .53 & & & & \\
\hline $923 R$ & .53 & & & & \\
\hline 961 & .57 & & & & \\
\hline 990 & .54 & & & & \\
\hline
\end{tabular}

\begin{tabular}{|c|c|c|c|}
\hline \multicolumn{4}{|c|}{ [Descriptive Statistics] } \\
\hline & TAN CH 2 & TAN CH 1 & All cores \\
\hline Parameter & TCP/MS & TCP/MS & ICPMS \\
\hline Minimum & 0.36 & 0.40 & 0.36 \\
\hline Maximum & .75 & .89 & .89 \\
\hline Mean & .56 & .55 & .55 \\
\hline Error & .01 & .03 & .01 \\
\hline Median & .54 & .505 & .53 \\
\hline Error & .02 & .04 & .02 \\
\hline Sample size & 35 & 18 & 56 \\
\hline
\end{tabular}


AA: Uranium (U)-Average crustal abundance is $2.3 \mathrm{ppm}$ (Emsley, 1989)

\begin{tabular}{|c|c|c|c|c|c|}
\hline \multicolumn{6}{|c|}{ [Concentration] } \\
\hline Depth & ICP/MS & Depth & ICPMS & Depth & ICP/MS \\
\hline 138 & 0.71 & 61 & 0.69 & 291 & 0.38 \\
\hline 154 & .67 & 91 & .57 & 450 & .10 \\
\hline 172 & .55 & 111 & .38 & 527 & .24 \\
\hline 201 & .51 & 130 & .37 & & \\
\hline 215 & .56 & 150 & .52 & & \\
\hline 238 & .45 & 195 & .49 & & \\
\hline 256 & .58 & $195 R$ & .46 & & \\
\hline 277 & .39 & 250 & .38 & & \\
\hline 299 & .53 & 300 & .33 & & \\
\hline 309 & .38 & 380 & .25 & & \\
\hline 327 & .37 & 421 & .26 & & \\
\hline 352 & .37 & 435 & .23 & & \\
\hline 366 & .30 & 460 & .34 & & \\
\hline 413 & .30 & 475 & .32 & & \\
\hline 463 & .19 & 491 & .35 & & \\
\hline 477 & .25 & 500 & .31 & & \\
\hline 487 & .35 & 521 & .24 & & \\
\hline 490 & .32 & 571 & .25 & & \\
\hline 521 & .39 & 593 & .25 & & \\
\hline 545 & .33 & $593 R$ & .23 & & \\
\hline 576 & .25 & & & & \\
\hline 592 & .23 & & & & \\
\hline 630 & .23 & & & & \\
\hline 659 & .25 & & & & \\
\hline 673 & .26 & & & & \\
\hline 730 & .28 & & & & \\
\hline 752 & .34 & & & & \\
\hline 785 & .22 & & & & \\
\hline 816 & .25 & & & & \\
\hline 832 & .29 & & & & \\
\hline 870 & .29 & & & & \\
\hline 886 & .38 & & & & \\
\hline 923 & .41 & & & & \\
\hline $923 \mathrm{R}$ & .36 & & & & \\
\hline 961 & .76 & & & & \\
\hline 990 & .37 & & & & \\
\hline \multicolumn{6}{|c|}{ [Descriptive Statistics] } \\
\hline & & TAN CH 2 & TAN CH 1 & All cores & \\
\hline & Parameter & ICP/MS & ICP/MS & ICP/MS & \\
\hline & Minimum & 0.19 & 0.23 & 0.10 & \\
\hline & Maximum & .76 & .69 & .76 & \\
\hline & Mean & .38 & .36 & .37 & \\
\hline & Error & .02 & .03 & .02 & \\
\hline & Median & .35 & .335 & .34 & \\
\hline & Error & .03 & .04 & .02 & \\
\hline & Sample size & 35 & 18 & 56 & \\
\hline
\end{tabular}


BB: Vanadium (V)—Average crustal abundance is 136 ppm (Emsley, 1989)

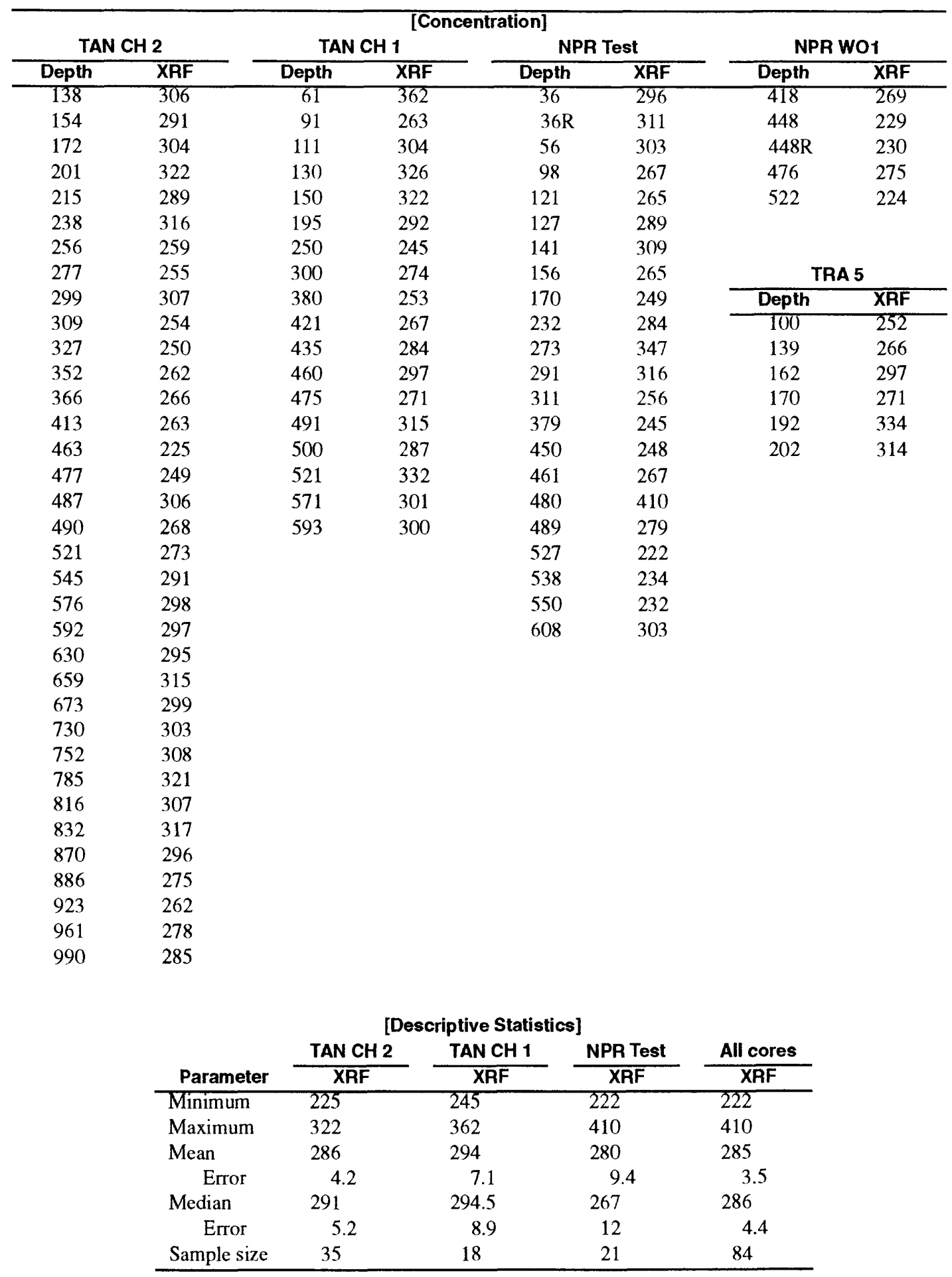


CC: Ytterbium (Yb) - Average crustal abundance is $3.1 \mathrm{ppm}$ (Emsley, 1989)

\begin{tabular}{|c|c|c|c|c|c|}
\hline \multicolumn{6}{|c|}{ [Concentration] } \\
\hline Depth & ICP/MS & Depth & ICP/MS & Depth & ICP/MS \\
\hline 138 & 4.5 & 61 & 5.7 & 291 & 3.0 \\
\hline 154 & 4.3 & 91 & 4.3 & 450 & 2.5 \\
\hline 172 & 4.2 & 111 & 3.9 & 527 & 2.4 \\
\hline 201 & 4.0 & 130 & 4.1 & & \\
\hline 215 & 4.1 & 150 & 4.4 & & \\
\hline 238 & 4.0 & 195 & 3.8 & & \\
\hline 256 & 3.5 & $195 \mathrm{R}$ & 3.7 & & \\
\hline 277 & 3.2 & 250 & 3.3 & & \\
\hline 299 & 4.0 & 300 & 3.2 & & \\
\hline 309 & 3.3 & 380 & 2.8 & & \\
\hline 327 & 3.2 & 421 & 3.0 & & \\
\hline 352 & 3.3 & 435 & 2.6 & & \\
\hline 366 & 3.0 & 460 & 3.1 & & \\
\hline 413 & 3.1 & 475 & 3.1 & & \\
\hline 463 & 2.3 & 491 & 3.4 & & \\
\hline 477 & 2.9 & 500 & 3.1 & & \\
\hline 487 & 3.2 & 521 & 3.1 & & \\
\hline 490 & 3.0 & 571 & 3.2 & & \\
\hline 521 & 3.6 & 593 & 3.1 & & \\
\hline 545 & 3.2 & 593R & 3.0 & & \\
\hline 576 & 3.0 & & & & \\
\hline 592 & 3.2 & & & & \\
\hline 630 & 3.0 & & & & \\
\hline 659 & 3.2 & & & & \\
\hline 673 & 3.4 & & & & \\
\hline 730 & 3.1 & & & & \\
\hline 752 & 3.8 & & & & \\
\hline 785 & 3.5 & & & & \\
\hline 816 & 3.6 & & & & \\
\hline 832 & 3.6 & & & & \\
\hline 870 & 3.4 & & & & \\
\hline 886 & 3.5 & & & & \\
\hline 923 & 3.3 & & & & \\
\hline $923 R$ & 3.3 & & & & \\
\hline 961 & 3.7 & & & & \\
\hline 990 & 3.5 & & & & \\
\hline \multicolumn{6}{|c|}{ [Descriptive Statistics] } \\
\hline & & TAN $\mathrm{CH}_{2}$ & TAN CH 1 & All co & \\
\hline & Parameter & ICP/MS & ICP/MS & ICP/ & \\
\hline & Minimum & 2.3 & 2.6 & 2.3 & \\
\hline & Maximum & 4.5 & 5.7 & 5.7 & \\
\hline & Mean & 3.5 & 3.5 & 3.4 & \\
\hline & Error & .08 & .18 & .0 & \\
\hline & Median & 3.4 & 3.2 & 3.3 & \\
\hline & Error & .10 & .22 & .1 & \\
\hline & Sample size & 35 & 18 & 56 & \\
\hline
\end{tabular}


DD: Yttrium (Y)—Average crustal abundance is $31 \mathrm{ppm}$ (Emsley, 1989)

\begin{tabular}{|c|c|c|c|c|c|c|c|c|c|c|c|}
\hline \multicolumn{12}{|c|}{ [Concentration] } \\
\hline \multicolumn{3}{|c|}{ TAN CH 2} & \multicolumn{4}{|c|}{ TAN CH 1} & \multicolumn{3}{|c|}{ NPR Test } & \multicolumn{2}{|c|}{ NPR W01 } \\
\hline Depth & ICP/MS & XRF & Depth & ICP/MS & XRF & & Depth & ICP/MS & $\overline{\mathrm{XRF}}$ & Depth & XRF \\
\hline 138 & 59 & 52 & 61 & 71 & 64 & & 36 & $\mathrm{ND}$ & 40 & 418 & 26 \\
\hline 172 & 52 & 47 & 111 & 48 & 45 & & 56 & ND & 40 & $448 \mathrm{R}$ & 23 \\
\hline 201 & 51 & 44 & 130 & 52 & 46 & & 98 & ND & 41 & 476 & 41 \\
\hline 215 & 52 & 47 & 150 & 54 & 48 & & 121 & ND & 34 & 522 & 23 \\
\hline 256 & 44 & 41 & $195 \mathrm{R}$ & 46 & ND & & 141 & ND & 42 & & \\
\hline 277 & 41 & 38 & 250 & 40 & 36 & & 156 & ND & 40 & & \\
\hline 299 & 49 & 46 & 300 & 39 & 35 & & 170 & ND & 39 & Depth & $\overline{X R F}$ \\
\hline 309 & 39 & 37 & 380 & 33 & 30 & & 232 & ND & 40 & 100 & 30 \\
\hline 327 & 39 & 35 & 421 & 36 & 33 & & 273 & ND & 44 & 139 & 31 \\
\hline 352 & 38 & 38 & 435 & 32 & 29 & & 291 & 37 & 35 & 162 & 32 \\
\hline 477 & 32 & 32 & 500 & 39 & 35 & & 461 & ND & 39 & & \\
\hline 487 & 39 & 37 & 521 & 36 & 33 & & 480 & ND & 62 & & \\
\hline 490 & 38 & 36 & 571 & 37 & 36 & & 489 & ND & 40 & & \\
\hline 521 & 45 & 41 & 593 & 37 & 34 & & 527 & 27 & 24 & & \\
\hline 545 & 40 & 38 & 593R & 36 & ND & & 538 & ND & 27 & & \\
\hline 576 & 36 & 34 & & & & & 550 & ND & 25 & & \\
\hline 592 & 40 & 36 & & & & & 608 & ND & 35 & & \\
\hline 630 & 36 & 34 & & & & & & & & & \\
\hline 659 & 39 & 36 & & & & & & & & & \\
\hline 673 & 38 & 37 & & & & & & & & & \\
\hline 730 & 37 & 35 & & & & & & & & & \\
\hline $923 R$ & 40 & ND & & & & & & & & & \\
\hline 961 & 45 & 40 & & & & & & & & & \\
\hline 990 & 42 & 39 & & & & & & & & & \\
\hline \multicolumn{12}{|c|}{ [Descriptive Statistics] } \\
\hline & & \multicolumn{2}{|c|}{ TAN CH 2} & \multicolumn{4}{|c|}{ TAN CH 1} & NPR Test & \multicolumn{2}{|c|}{ All cores } & \\
\hline & Parameter & ICP/MS & $\overline{X R F}$ & & MS & $\overline{X R F}$ & & XRF & ICP/MS & XRF & \\
\hline & Minimum & 26 & 25 & 3 & & 29 & & 24 & 26 & 23 & \\
\hline & Maximum & 59 & 52 & 7 & & 64 & & 62 & 71 & 64 & \\
\hline & Mean & 42 & 39 & 4 & & 39 & & 37 & 42 & 38 & \\
\hline & Error & 1.1 & 0.9 & & & 2 & & 1.8 & 1.1 & 0.83 & \\
\hline & Median & 41 & 38 & 3 & & 36 & & 39 & 40 & 37 & \\
\hline & Error & 1.4 & 1.1 & & & 2.5 & & 2.3 & 1.4 & 1.0 & \\
\hline & Sample size & 35 & 35 & 1 & & 18 & & 21 & 56 & 84 & \\
\hline
\end{tabular}


EE: Zinc (Zn)-Average crustal abundance is $76 \mathrm{ppm}$ (Emsley, 1989)

\begin{tabular}{|c|c|c|c|c|c|c|c|}
\hline \multicolumn{8}{|c|}{ [Concentration] } \\
\hline \multicolumn{2}{|c|}{ TAN CH 2} & \multicolumn{2}{|c|}{ TAN CH 1} & \multicolumn{2}{|c|}{ NPR Test } & \multicolumn{2}{|c|}{ NPR W01 } \\
\hline Depth & $\overline{X R F}$ & Depth & XRF & Depth & XRF & Depth & XRF \\
\hline 138 & 150 & 61 & 158 & 36 & 123 & 418 & 75 \\
\hline 154 & 145 & 91 & 139 & $36 \mathrm{R}$ & 124 & 448 & 76 \\
\hline 172 & 143 & 111 & 141 & 56 & 121 & $448 \mathrm{R}$ & 68 \\
\hline 201 & 142 & 130 & 145 & 98 & 117 & 476 & 125 \\
\hline 215 & 141 & 150 & 140 & 121 & 98 & 522 & 77 \\
\hline 238 & 141 & 195 & 133 & 127 & 104 & & \\
\hline 256 & 126 & 250 & 122 & 141 & 126 & & \\
\hline 277 & 125 & 300 & 119 & 156 & 112 & \multicolumn{2}{|c|}{ TRA 5} \\
\hline 299 & 141 & 380 & 104 & 170 & 115 & Depth & XRF \\
\hline 309 & 126 & 421 & 110 & 232 & 120 & 100 & 96 \\
\hline 327 & 121 & 435 & 103 & 273 & 129 & 139 & 98 \\
\hline 352 & 113 & 460 & 129 & 291 & 115 & 162 & 98 \\
\hline 366 & 117 & 475 & 125 & 311 & 100 & 170 & 98 \\
\hline 413 & 114 & 491 & 132 & 379 & 90 & 192 & 126 \\
\hline 463 & 95 & 500 & 130 & 450 & 74 & 202 & 131 \\
\hline 477 & 100 & 521 & 126 & 461 & 129 & & \\
\hline 487 & 136 & 571 & 124 & 480 & 155 & & \\
\hline 490 & 128 & 593 & 123 & 489 & 127 & & \\
\hline 521 & 151 & & & 527 & 77 & & \\
\hline 545 & 134 & & & 538 & 74 & & \\
\hline 576 & 128 & & & 550 & 76 & & \\
\hline 592 & 127 & & & 608 & 107 & & \\
\hline 630 & 126 & & & & & & \\
\hline 659 & 129 & & & & & & \\
\hline 673 & 125 & & & & & & \\
\hline 730 & 129 & & & & & & \\
\hline 752 & 140 & & & & & & \\
\hline 785 & 140 & & & & & & \\
\hline 816 & 142 & & & & & & \\
\hline 832 & 139 & & & & & & \\
\hline 870 & 136 & & & & & & \\
\hline 886 & 126 & & & & & & \\
\hline 923 & 121 & & & & & & \\
\hline 961 & 127 & & & & & & \\
\hline 990 & 129 & & & & & & \\
\hline
\end{tabular}

[Descriptive Statistics]

\begin{tabular}{|c|c|c|c|c|}
\hline & TAN CH 2 & TAN CH 1 & NPR Test & All cores \\
\hline Parameter & XRF & XRF & XRF & XRF \\
\hline Minimum & 95 & 103 & 74 & 74 \\
\hline Maximum & 151 & 158 & 155 & 158 \\
\hline Mean & 130 & 128 & 109 & 121 \\
\hline Error & 2.1 & 3.3 & 4.7 & 2.2 \\
\hline Median & 129 & 127.5 & 115 & 126 \\
\hline Error & 2.7 & 4.2 & 5.9 & 2.7 \\
\hline Sample size & 35 & 18 & 21 & 84 \\
\hline
\end{tabular}


FF: Zirconium (Zr) -Average crustal abundance is $162 \mathrm{ppm}$ (Emsley, 1989)

\begin{tabular}{|c|c|c|c|c|c|c|c|}
\hline \multicolumn{8}{|c|}{ [Concentration] } \\
\hline \multicolumn{2}{|c|}{ TAN CH 2} & \multicolumn{2}{|c|}{ TAN CH 1} & \multicolumn{2}{|c|}{ NPR Test } & \multicolumn{2}{|c|}{ NPR W01 } \\
\hline Depth & XRF & Depth & XRF & Depth & $\overline{\mathbf{X R F}}$ & Depth & $\overline{\text { XRF }}$ \\
\hline 138 & 327 & 61 & 409 & 36 & 240 & 418 & 108 \\
\hline 154 & 308 & 91 & 293 & $36 \mathrm{R}$ & 239 & 448 & 94 \\
\hline 172 & 294 & 111 & 285 & 56 & 232 & $448 \mathrm{R}$ & 95 \\
\hline 201 & 286 & 130 & 299 & 98 & 247 & 476 & 237 \\
\hline 215 & 309 & 150 & 305 & 121 & 178 & 522 & 109 \\
\hline 238 & 287 & 195 & 263 & 127 & 183 & & \\
\hline 256 & 256 & 250 & 214 & 141 & 252 & & \\
\hline 277 & 222 & 300 & 211 & 156 & 257 & \multicolumn{2}{|c|}{ TRA 5} \\
\hline 299 & 272 & 380 & 155 & 170 & 252 & Depth & $\overline{\mathbf{X R F}}$ \\
\hline 309 & 218 & 421 & 183 & 232 & 261 & 100 & 169 \\
\hline 327 & 204 & 435 & 162 & 273 & 270 & 139 & 164 \\
\hline 352 & 213 & 460 & 211 & 291 & 211 & 162 & 157 \\
\hline 366 & 194 & 475 & 215 & 311 & 175 & 170 & 144 \\
\hline 413 & 197 & 491 & 226 & 379 & 151 & 192 & 285 \\
\hline 463 & 124 & 500 & 214 & 450 & 104 & 202 & 297 \\
\hline 477 & 161 & 521 & 173 & 461 & 237 & & \\
\hline 487 & 226 & 571 & 186 & 480 & 375 & & \\
\hline 490 & 211 & 593 & 183 & 489 & 236 & & \\
\hline 521 & 247 & & & 527 & 109 & & \\
\hline 545 & 221 & & & 538 & 109 & & \\
\hline 576 & 182 & & & 550 & 107 & & \\
\hline 592 & 195 & & & 608 & 189 & & \\
\hline 630 & 181 & & & & & & \\
\hline 659 & 194 & & & & & & \\
\hline 673 & 204 &. & & & & & \\
\hline 730 & 191 & & & & & & \\
\hline 752 & 270 & & & & & & \\
\hline 785 & 228 & & & & & & \\
\hline 816 & 240 & & & & & & \\
\hline 832 & 239 & & & & & & \\
\hline 870 & 234 & & & & & & \\
\hline 886 & 248 & & & & & & \\
\hline 923 & 240 & & & & & & \\
\hline 961 & 259 & & & & & & \\
\hline 990 & 207 & & & & & & \\
\hline \multicolumn{8}{|c|}{ [Descriptive Statistics] } \\
\hline & & TAN & & TAN CH 1 & NPR Test & All cores & \\
\hline & Parameter & & & $\overline{\mathbf{X R F}}$ & XRF & XRF & \\
\hline & Minimum & & & 155 & 104 & 94 & \\
\hline & Maximum & & & 409 & 375 & 409 & \\
\hline & Mean & & & 233 & 208 & 219 & \\
\hline & Error & & & 15 & 15 & 6.7 & \\
\hline & Median & & & 214 & 232 & 216.5 & \\
\hline & Error & & & 19 & 19 & 8.3 & \\
\hline & Sample size & & & 18 & 21 & 84 & \\
\hline
\end{tabular}

